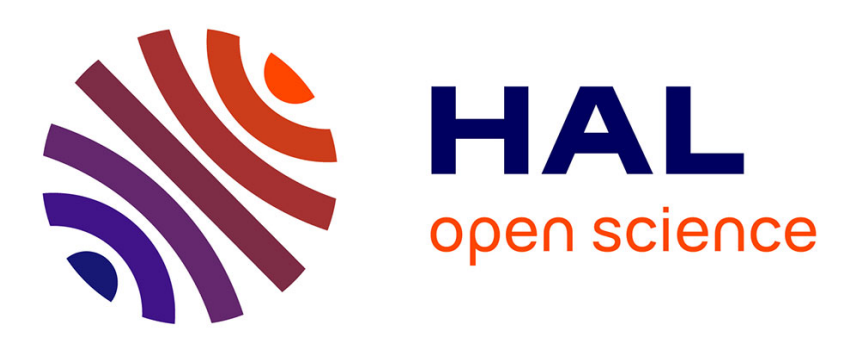

\title{
Activation of enzymatically produced chitooligosaccharides by dioxyamines and dihydrazides
}

Ingrid Vikøren Mo, Yiming Feng, Marianne Øksnes Dalheim, Amalie Solberg, Finn L. Aachmann, Christophe Schatz, Bjørn E. Christensen

\section{- To cite this version:}

Ingrid Vikøren Mo, Yiming Feng, Marianne Øksnes Dalheim, Amalie Solberg, Finn L. Aachmann, et al.. Activation of enzymatically produced chitooligosaccharides by dioxyamines and dihydrazides. Carbohydrate Polymers, 2020, 232, pp.115748. 10.1016/j.carbpol.2019.115748 . hal-02530252

\section{HAL Id: hal-02530252 \\ https://hal.science/hal-02530252}

Submitted on 6 Apr 2020

HAL is a multi-disciplinary open access archive for the deposit and dissemination of scientific research documents, whether they are published or not. The documents may come from teaching and research institutions in France or abroad, or from public or private research centers.
L'archive ouverte pluridisciplinaire HAL, est destinée au dépôt et à la diffusion de documents scientifiques de niveau recherche, publiés ou non, émanant des établissements d'enseignement et de recherche français ou étrangers, des laboratoires publics ou privés. 


\section{Activation of enzymatically produced chitooligosaccharides by dioxyamines and dihydrazides}

Ingrid Vikøren $\mathrm{Mo}^{[\mathrm{a}]}$, Yiming Feng ${ }^{[a]}$, Marianne Øksnes Dalheim ${ }^{[a]}$, Amalie Solberg ${ }^{[a]}$, Finn L. Aachmann ${ }^{[a]}$, Christophe Schatz ${ }^{*[b]}$, and Bjørn E. Christensen*[a]

${ }^{[a]}$ NOBIPOL, Department of Biotechnology and Food Science, NTNU Norwegian University of Science and Technology, Sem Sælands vei 6/8, NO-7491 Trondheim, Norway, E-mail: ingrid.v.mo@ntnu.no, yiming.feng@yahoo.com, marianne.dalheim@rise-pfi.no, amalie.solberg@ntnu.no, finn.l.aachmann@ntnu.no, bjorn.e.christensen@ntnu.no

${ }^{[b]}$ LCPO, Université de Bordeaux, UMR5629, ENSCBP, 16, Avenue Pey Berland, 33607 Pessac Cedex, France, E-mail: schatz@enscbp.fr

${ }^{*}$ Corresponding authors: Bjørn E. Christensen, bjorn.e.christensen@ntnu.no, +47 73593327

Christophe Schatz, schatz@enscbp.fr, +33556846618

This manuscript is dedicated to memory of Professor Kjell M. Vårum 
Abstract: Reducing end activation of poly- and oligosaccharides by bifunctional dioxyamines and dihydrazides enables aniline-free and cyanoborohydride-free conjugation to aldehyde-containing molecules, particles and surfaces without compromising the chain structure. Chitosans are due to their polycationic character, biodegradability, and bioactivity important candidates for conjugation. Here, we present a kinetic and structural study of the conjugation of a dioxyamine and a dihydrazide to enzymatically produced chitooligosaccharides ranging from $N, N$ '-diacetylchitobiose to a decamer, all having $N$-acetyl D-glucosamine at the reducing end. Conjugation of the dioxyamine resulted in mixtures of $(E)$ - and $(Z)$-oximes and $\beta-N$ pyranoside, whereas the dihydrazide yielded cyclic $N$-glycosides. Reaction kinetics was essentially independent of DP. Stable secondary amines were in both cases obtained by reduction with a-picoline borane, but higher temperatures were needed to obtain acceptable reduction rate. Comparison to dextran oligomers shows that the nature of the reducing end strongly influences the kinetics of both the conjugation and reduction.

Keywords: carbohydrates $\bullet$ chitosan $\bullet$ conjugation $\bullet$ hydrazides $\bullet$ oxyamines

\section{Introduction}

Chemical modification of carbohydrates is commonly performed to change their properties for various applications. However, optimal retention of their intrinsic properties requires reactions at the chain termini (terminal conjugation) rather than traditional lateral substitution. Reaction at the reducing end is the dominating method for terminal modification (Novoa-Carballal \& Müller, 2012; Schatz \& Lecommandoux, 2010), where the hemiacetal-aldehyde equilibrium enables aldehyde-based reactions for the conjugation to other molecules, particles and surfaces. Among chemical modification methods, alkyne/azide click chemistry (Breitenbach, Schmid, \& Wich, 2017; Rosselgong et al., 2019), thioacetylation (Pickenhahn et al., 2015), and reductive amination using oximes (Novoa-Carballal \& Müller, 2012) or hydrazones (Kölmel \& Kool, 2017) are the most common. It may be noted that the alkyne/azide click chemistry approach also depends on an initial reductive amination step with alkyne- or azide-bearing amines.

Amines, oxyamines and hydrazides can react with aldehydes to form Schiff bases (imines, oximes and hydrazones), normally combined with subsequent reduction to form stable secondary amines (Figure 1). Oxyamines and hydrazides have higher nucleophilicities compared to amines (Fina \& Edwards, 1973), and can efficiently conjugate to the reducing end of carbohydrates (Baudendistel, Wieland, Schmidt, \& Wittmann, 2016; Hermanson, 2008; Kwase, Cochran, \& Nitz, 2013; Lee \& Shin, 2005; Novoa-Carballal \& Müller, 2012). They also increase the hydrolytic stability of the formed Schiff bases (oximes and hydrazones), and their low basicity allows them to form under acidic conditions (Kalia \& Raines, 2008). Oxyamines tend to form acyclic oximes with carbohydrates, both in the $(E)$ - and (Z)-configuration, in equilibrium with cyclic $N$-glycosides (Figure 1)(Kwase et al., 2013). The relative distribution of conjugates depends on the chemistry of the reducing end residue (Baudendistel et al., 2016). Hydrazides, on the other hand, predominantly form $\mathrm{N}$ glycosides when conjugated under acidic conditions (Lee \& Shin, 2005; Shinohara et al., 1996). Hence, oxyamines and hydrazides can be used to form $\mathrm{pH}$-sensitive carbohydrate-based conjugates or fully stable conjugates by reduction to secondary amines. 
Aniline may be an effective catalyst in reactions with both amines (Guerry et al., 2013), oxyamines (Dirksen, Hackeng, \& Dawson, 2006; Thygesen et al., 2010) and hydrazides (Dirksen \& Dawson, 2008). It can, however, give side products (Guerry et al., 2013; Thygesen et al., 2010) and its toxicity may raise HSE and environmental concerns. Hence, aniline-free protocols are sometimes preferred.

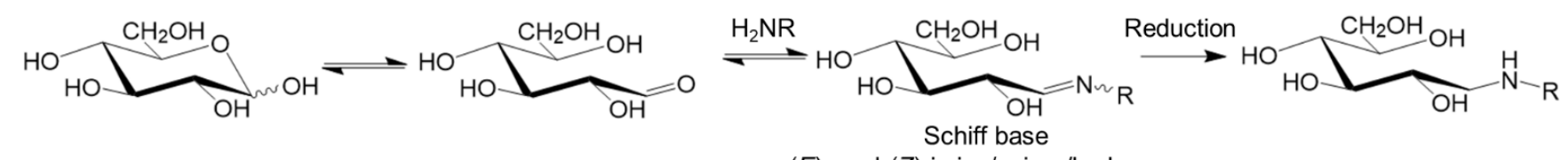

$(E)$ - and (Z)-imine/oxime/hydrazone

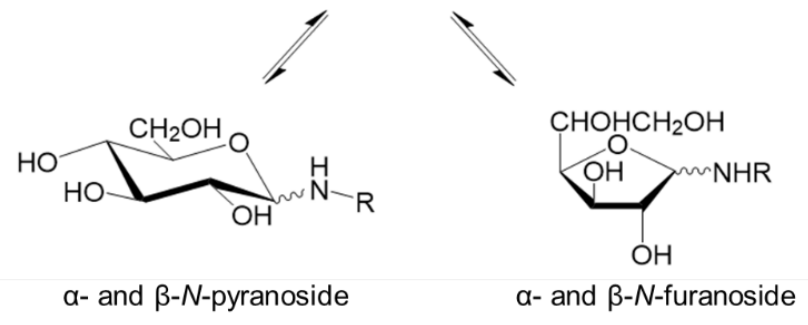

Figure 1. Reaction of the free aldehyde at the reducing end of carbohydrates with amines and the subsequent reduction of the Schiff bases to form stable secondary amines. D-glucopyranose is here shown as example.

Chitin is a highly abundant polysaccharide, found in e.g. the exoskeleton of crustaceans, insects, squid pens, and in the cell wall of some bacteria and fungi. It is a high molecular weight, crystalline, water-insoluble polymer consisting exclusively of 1,4-linked $\mathrm{N}$-acetyl-D-glucosamine (GlcNAc, A) residues. Partial de- $\mathrm{N}$ acetylation of chitin provides chitosan with varying amounts of D-glucosamine (GIcN, D) (Figure 2a). Chitosans are commonly described by the fraction of residual $A$ residues $\left(F_{A}\right)$ (Vårum, Antohonsen, Grasdalen, \& Smidsrød, 1991) following de- $N$-acetylation (Supporting Information, S1). Chitosans are the only polycationic polysaccharides which can be isolated from biomass and they are widely studied because of their ( $\mathrm{pH}$ dependent) cationic properties, which can be utilised in polyelectrolyte complexes with e.g. DNA for transfection (Strand, Danielsen, Christensen, \& Vårum, 2005), antimicrobial activity (Mellegård, Strand, Christensen, Granum, \& Hardy, 2011), bioadhesive properties biocompatibility, and biodegradability.

a)

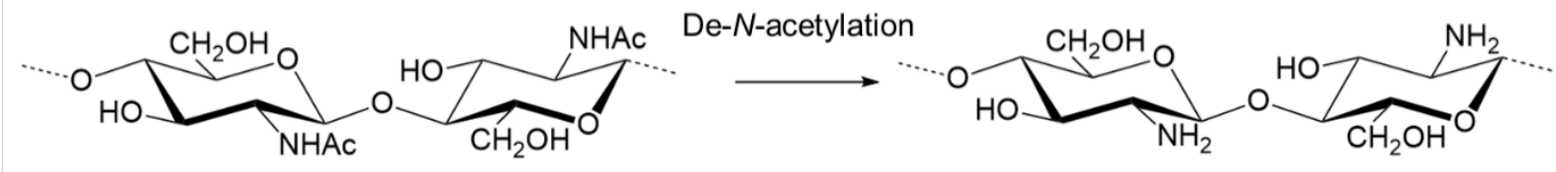

$N$-acetyl-D-glucosamine (A)

D-glucosamine (D)

b)<smiles>NNC(=O)CCCCC(=O)NN</smiles>

PDHA

$\mathrm{ADH}$<smiles></smiles>

PB

Figure 2. a) Conversion of chitin to chitosan by partial or complete de- $N$-acetylation. Residues are conveniently abbreviated $A(N$-acetyl$D$-glucosamine) and $D$ (D-glucosamine). The fraction of unmodified $A$ residues $\left(F_{A}\right)$ is used for characterization of the chitosan, and 
complete de- $N$-acetylation, as shown, gives $F_{A}=0$. b) Structures of $O, O^{\prime}-1,3$-propanediylbishydroxylamine (PDHA), adipic acid dihydrazide $(\mathrm{ADH})$ and $\alpha$-picoline borane $(\mathrm{PB})$

Nitrous acid degradation of chitosan is a standard route to prepare chitooligosaccharides with a reactive 2,5anhydro D-mannose (M) residue at the reducing end (Allan \& Peyron, 1995; Moussa, Crepet, Ladaviere, \& Trombotto, 2019; Tømmeraas, Vårum, Christensen, \& Smidsrød, 2001). However, oligomers of the type $D_{n} M$ (where $\mathrm{n}$ is the number of contiguous uninterrupted $\mathrm{D}$-units) can only be obtained from $100 \%$ de- $N$-acetylated chitosans, which require extensive de- $N$-acetylation. In contrast, chitooligosaccharides of the type $D_{n} X A$ (where $X$ is either $D$ or $A$ ) may me more relevant from a biorefinery point of view because they can be obtained by enzymatic degradation of chitosans using specific chitinases such as chitinase $B(C h i B)$ from Serratia marcescens (Sørbotten, Horn, Eijsink, \& Vårum, 2005). The uninterrupted $D_{n}$ sequences allow for cooperative, preferentially electrostatic, interactions with other molecules, in contrast to sequences with randomly positioned $A$ residues. Residues of $A$ at the reducing end are also preferred over $D$ residues because of the poorer reactivity of the latter (Beaudoin, Gauthier, Boucher, \& Waldron, 2005; Guerry et al., 2013). Further, the $\mathrm{N}$-acetyl group of the terminal A residue provide a particularly useful marker in chemical analyses, besides allowing direct comparison to previous work based on monomeric $A$ and the AA dimer (Baudendistel et al., 2016).

In the present work, we study in detail the conjugation of a dioxyamine, $O, O^{\prime}-1,3-$ propanediylbishydroxylamine (PDHA, Figure $2 b$ ) and a dihydrazide, adipic acid dihydrazide (ADH, Figure $2 b$ ) to $D_{n} X A$ chitooligosaccharides. All steps in the conjugation (amination) reactions with oxyamines and hydrazides are reversible, and the stability of the conjugates may vary considerably (Kwase et al., 2013). We have therefore further studied the oxime/hydrazone reduction using $\alpha$-picoline borane (PB, Figure $2 b$ ). PB is a relatively new and so far little described reducing agent, introduced as a green and less hazardous alternative to sodium cyanoborohydride $\left(\mathrm{NaCNBH}_{3}\right)$ (Cosenza, Navarro, \& Stortz, 2011; Dalheim et al., 2016). PB has been applied for labelling of oligosaccharides and glycans by reductive amination, leading to equal or even better reducing efficacies compared to $\mathrm{NaCNBH}_{3}$ (Cosenza et al., 2011; Fang, Qin, Ma, \& She, 2015; Ruhaak, Steenvoorden, Koeleman, Deelder, \& Wuhrer, 2010; Ruhaak, Zauner, et al., 2010; Unterieser \& Mischnick, 2011). Moreover, PB can be employed under both aqueous and non-aqueous conditions, which is advantageous for reactions with carbohydrates(Ruhaak, Zauner, et al., 2010). Reduction is also a means to tailor the properties of the conjugates, as charge/pK $\mathrm{K}_{\mathrm{a}}$ and local flexibility are generally different for the reduced and unreduced forms.

Here, we show that chitooligosaccharides react in rather different ways with PDHA and ADH, both in terms of kinetics, yields and selectivity. The same applies to the reduction by PB. We also show that the rate of conjugation and reduction depend significantly on the nature of the reducing end residue by comparing the chitooligosaccharides to dextran oligomers. Taken together the findings provide a solid basis for selecting optimal aniline-free and cyanoborohydride-free protocols for the preparation of dioxyamine and dihydrazide activated chitooligosaccharides (with and without reduction).

\section{Experimental Section}

\subsection{Materials}


The chitosan $\left(F_{A}=0.25,[\eta]=420 \mathrm{~g} / \mathrm{mL}\right)$ was an in-house sample prepared by de- $N$-acetylation of chitin. Chitinase B (ChiB, Serratia marcescens) was kindly provided by Prof. Vincent G. H. Eijsink, NMBU, Norway. $N, N$ '-diacetyl chitobiose (AA), adipic acid dihydrazide (ADH), O,O'-1,3-propanediylbishydroxylamine dihydrochloride (PDHA) and 2-methylpyridine borane complex ( $\alpha$-picoline borane, $\mathrm{PB}$ ) were obtained from Sigma-Aldrich. All other chemicals were obtained from commercial sources and were of analytical grade.

\subsection{Preparative gel filtration chromatography (GFC)}

The preparative gel filtration system was composed of two Superdex 30 (prep. grade) columns (BPG 140/950, $140 \mathrm{~mm}$ x $95 \mathrm{~cm}$, GE Healthcare Life Sciences) connected in series, continuously eluting ammonium acetate $(\mathrm{AmAc})$ buffer $(0.15 \mathrm{M}, \mathrm{pH} 4.5)$ with a flow rate of $30 \mathrm{~mL} / \mathrm{min}$. Samples $(0.5-3 \mathrm{~g})$ were dissolved in buffer and injected into the system. The fractionation was monitored by an on-line refractive index (RI) detector (SHODEX R1-102). Fractions (160 mL/flask) were collected (Super Frac, Amersham Biosciences) and pooled according to elution times. The pooled fractions were reduced to appropriate volumes, dialyzed (MWCO = 100-500) against MQ-water until the measured conductivity of the water was $<2 \mu \mathrm{S} / \mathrm{cm}$ and freeze-dried.

\subsection{Analytical gel filtration chromatography (GFC)}

The analytical gel filtration system was composed of three Superdex 30 (prep. grade) columns (HiLoad 26/60, $26 \mathrm{~mm} \times 60 \mathrm{~cm}$, GE Healthcare Life Sciences) connected in series, continuously eluting AmAc buffer (0.15 M, $\mathrm{pH} 4.5$ or $0.1 \mathrm{M}, \mathrm{pH} 6.9)$ with a flow rate of $0.8 \mathrm{~mL} / \mathrm{min}$. Samples $(5-100 \mathrm{mg})$ were dissolved in buffer and injected into the system. The fractionation was monitored by an on-line RI-detector (SHODEX R1-101). Fractions (3.2 mL/tube) were collected (LKB 2111 Multirack KS1) and pooled according to elution times. The pooled fractions were reduced to appropriate volumes, dialyzed ( $M W C O=100-500)$ against $M Q$-water until the measured conductivity of the water was $<2 \mu \mathrm{S} / \mathrm{cm}$ and freeze-dried.

\subsection{NMR spectroscopy}

Samples were dissolved in $\mathrm{D}_{2} \mathrm{O}(450-600 \mu \mathrm{L}$, approx. $10 \mathrm{mg} / \mathrm{mL})$ and adjusted to target $\mathrm{pD}$ with $\mathrm{DCl} / \mathrm{NaOD}$. For some samples, $1 \%$ sodium 3-(trimethylsilyl)-propionate- $d_{4}(\mathrm{TSP}, 3 \mu \mathrm{L})$ was added as an internal standard. Samples for the time course NMR experiments were prepared in deuterated NaAc-buffer $(500 \mathrm{mM}, \mathrm{pH}=3.0$, 4.0 or $5.0,2 \mathrm{mM}$ TSP).

All homo and heteronuclear NMR experiments were carried out on a Bruker Ascend $400 \mathrm{MHz}$, ultra-shielded $600 \mathrm{MHz}$ or Ascend $800 \mathrm{MHz}$ spectrometer (Bruker BioSpin AG, Fällanden, Switzerland) equipped with Avance III HD electronics and a $5 \mathrm{~mm}$ SmartProbe with z-gradients, a $5 \mathrm{~mm}$ cryogenic CP-QCl z-gradient probe or a $5 \mathrm{~mm}$ z-gradient CP-Cl cryogenic probe, respectively.

Characterisation of oligomers, purified conjugates or reaction mixtures were performed by obtaining $1 \mathrm{D}^{1} \mathrm{H}$ NMR spectra at $300 \mathrm{~K}$ on the $600 \mathrm{MHz}$ spectrometer. $\mathrm{pH}$ titration was performed by obtaining $1 \mathrm{D}{ }^{1} \mathrm{H}-\mathrm{NMR}$ spectra at different $\mathrm{pH}$ values at $298 \mathrm{~K}$ on the $400 \mathrm{MHz}$ spectrometer. Time course experiments were performed by obtaining $1 \mathrm{D}^{1} \mathrm{H}-\mathrm{NMR}$ spectra at specific time points at $300 \mathrm{~K}$ on the $600 \mathrm{MHz}$ spectrometer. Chemical shift assignments were performed at $298-310 \mathrm{~K}$ on the $800 \mathrm{MHz}$ spectrometer by obtaining the following homo and heteronuclear NMR spectra: $1 \mathrm{D}^{1} \mathrm{H}, 2 \mathrm{D}$ double quantum filtered correlation spectroscopy 
(DQF-COSY), 2D total correlation spectroscopy (TOCSY) with $70 \mathrm{~ms}$ mixing time, $2 \mathrm{D}^{13} \mathrm{C}$ heteronuclear single quantum coherence (HSQC) with multiplicity editing, $2 \mathrm{D}{ }^{13} \mathrm{C}$ Heteronuclear 2 Bond Correlation $(\mathrm{H} 2 \mathrm{BC}), 2 \mathrm{D}{ }^{13} \mathrm{C}$ HSQC- $\left[{ }^{1} \mathrm{H},{ }^{1} \mathrm{H}\right]$ TOCSY with $70 \mathrm{~ms}$ mixing time on protons and $2 \mathrm{D}$ heteronuclear multiple bond correlation (HMBC) with BIRD filter to suppress first order correlations.

All spectra were recorded, processed and analysed using TopSpin 3.5 software (Bruker BioSpin).

\subsection{Flow injection analysis (FIA) coupled with quadrupole time of flight (qTOF) MS}

The analyses were performed with an ACQUITY I-class UPLC system coupled to a Synapt G2Si HDMS mass spectrometer (Waters, Milford, MA, USA) equipped with an ESI source operating in negative or positive mode.

FIA analysis was performed by operating the UHPLC in bypass mode, directing the flow past the compartment directly to the mass spectrometer. A mobile phase consisting of $100 \%$ methanol and a programmed linear flow gradient was used. The flow rate was constant at $0.150 \mathrm{~mL} / \mathrm{min}$ for $0.10 \mathrm{~min}$, then reduced to $0.030 \mathrm{~min}$ until $1.50 \mathrm{~min}$, then increased to $0.200 \mathrm{~mL} / \mathrm{min}$ until $1.60 \mathrm{~min}$, and kept constant to $2.50 \mathrm{~min}$. Finally, the system was equilibrated for additionally $0.50 \mathrm{~min}$ at $0.150 \mathrm{~mL} / \mathrm{min}$ before the next injection. Total run time was $3.0 \mathrm{~min}$. The injection volume was set to $2 \mu \mathrm{L}$.

MS analysis was performed under constant ESI conditions. The capillary voltage, cone voltage and source offset voltage in negative and positive mode were set at $-2.0 \mathrm{kV} / 3.0 \mathrm{kV},-30 \mathrm{~V} / 30 \mathrm{~V}$ and $-40 \mathrm{~V} / 40 \mathrm{~V}$, respectively. The source temperature was maintained at $120^{\circ} \mathrm{C}$ and the desolvation gas temperature and desolvation gas flow was set to $500{ }^{\circ} \mathrm{C}$ and $800 \mathrm{~L} / \mathrm{h}$, respectively. The cone gas flow rate was fixed at $50 \mathrm{~L} / \mathrm{h}$ and the nebulizer gas flow was maintained at 6 bar. The instrument was operated in high-resolution mode with a cycle time of $1.015 \mathrm{~s}$, consisting of a scan time of $1 \mathrm{~s}$, and an inter-scan delay of $15 \mathrm{~ms}$. The mass range was set to 50-2000 Da, the same range as the valid calibration performed with Na-formate immediately before analysis.

A reference mass/lock mass consisting of leucine enkephalin $(1 \mathrm{ng} / \mathrm{mL})$ in $50 \%(\mathrm{v} / \mathrm{v})$ acetonitrile in water was infused into the ion source through a separate capillary at a flow rate of $10 \mu \mathrm{L} / \mathrm{min}$ to correct the mass axis on the fly. The capillary voltage of the lock mass was set to $2.5 \mathrm{kV}$ (negative or positive depending on the operation mode).

Data was acquired and processed using MassLynx software (v4.1).

\subsection{Preparation of chitooligosaccharides $\left(D_{n} X A, X=D / A\right)$}

Chitosan $\left(F_{A}=0.25\right)$ was dissolved in $M Q$ water (deionized water purified with the MilliQ system from Millipore, Bedford, MA, USA) by stirring overnight and added an equal volume of $\mathrm{NaAc}$-buffer $(0.2 \mathrm{M}, \mathrm{pH}=$ 5.5) to a final chitosan concentration of $10 \mathrm{mg} / \mathrm{mL}$. The chitosan solution was incubated for 1 hour in a shaking water bath $\left(60 \mathrm{rpm}, 37^{\circ} \mathrm{C}\right)$. ChiB was added to the chitosan solution $(4.3 \mu \mathrm{g} / \mathrm{mg}$ chitosan) and reacted in the shaking water bath for 7 days. The degradation was terminated by adjusting the $\mathrm{pH}$ to 2.5 using $\mathrm{HCl}(0.1 \mathrm{M})$ and boiling the solution for 5 min. Subsequently, $\mathrm{pH}$ was adjusted to $\mathrm{pH} 4.5$ using $\mathrm{NaOH}(0.1 \mathrm{M})$. The degradation mixture was filtrated $(0.22 \mu \mathrm{m})$ and freeze-dried. Chitosan oligomers were separated according to degree of polymerization (DP) using the preparative GFC system (0.15 M AmAc, pH 4.5). Pooled fractions 
from the GFC separation were dialyzed $(M W C O=100-500)$ against $M Q$-water to remove salt from the buffer until the measured conductivity was $<2 \mu \mathrm{S} / \mathrm{cm}$ and finally freeze-dried. Selected chitosan oligomers $\left(D_{n} X A, n\right.$ $=2$ and 3) were subsequently re-chromatographed using the analytical GFC system (0.1 M AmAc, pH 6.9). The oligomers were separated according to chemical composition $\left(D_{n} A A\right.$ or $\left.D_{n} D A\right)$ and purified by dialysis as described above. Purified oligomers were characterised by $1 \mathrm{D}^{1} \mathrm{H}-\mathrm{NMR}(600 \mathrm{MHz}$ spectrometer).

\section{$2.7^{1} \mathrm{H}-\mathrm{NMR}$ pH titration of PDHA and ADH}

ADH or PDHA was dissolved in 1:10 v/v $\mathrm{D}_{2} \mathrm{O}$ in $\mathrm{H}_{2} \mathrm{O}(500 \mu \mathrm{L}, 10 \mathrm{mg} / \mathrm{mL}) .1 \%$ TSP $(3 \mu \mathrm{L})$ was added as an internal standard. The $\mathrm{pH}$ was adjusted with $\mathrm{HCl}(0.1$ and $1.0 \mathrm{M})$ or $\mathrm{NaOH}(0.1$ and $1.0 \mathrm{M}) .{ }^{1} \mathrm{H}$-NMR spectra were obtained every 0.1 to $0.8 \mathrm{pH}$ unit in the $\mathrm{pH}$ range 6.1 to 0.5 (400 $\mathrm{MHz}$ spectrometer). The system was locked on the internal $\mathrm{D}_{2} \mathrm{O}$ and the spectra were recorded using water suppression.

\subsection{Conjugation and reduction studied by time course NMR}

Deuterated NaAc-buffer $(500 \mathrm{mM}, \mathrm{pH}=3.0,4.0$ or 5.0$)$ containing $2 \mathrm{mM}$ TSP was prepared by dissolving the required volumes of deuterated acetic acid $\left(\mathrm{CD}_{3} \mathrm{COOD}, 99.5 \% \mathrm{D}_{4}\right)$ and TSP (from a $20 \mathrm{mM}$ stock solution prepared using $\mathrm{D}_{2} \mathrm{O}$ ) in $\mathrm{D}_{2} \mathrm{O}$ (3/4 of total volume). The $\mathrm{pH}$ was adjusted with $\mathrm{NaOD}$ to the desired value and diluted with $\mathrm{D}_{2} \mathrm{O}$ to the final volume. As the $\mathrm{pH}$-meter used was calibrated with non-deuterated buffers, the $\mathrm{pH}$ reading, $\mathrm{pH}^{*}$, was converted to the real $\mathrm{pH}$ using the formula $\mathrm{pH}=0.9291 \times \mathrm{pH}^{*}+0.421$ (Baudendistel et al., 2016; Krȩżel \& Bal, 2004).

$N, N$ '-diacetyl chitobiose (AA), chitobiose (DD), chitooligosaccharides $\left(D_{n} X A\right)$ or dextran oligomers (Dext $)$ (20.1 mM) and 2 or 10 equivalents ADH/PDHA (40.2 or $201 \mathrm{mM}$ ) were dissolved separately in deuterated NaAc-buffer ( $500 \mathrm{mM}, \mathrm{pH}=3,4$ or 5 ) containing $2 \mathrm{mM}$ TSP and transferred to a $5 \mathrm{~mm}$ NMR tube. The mixing of the reagents in the NMR tube served as time zero $(t=0)$. The concentrations given in parenthesis represents final concentrations after mixing, which also apply in all the following sections. For the reduction experiments, 3 equivalents $(60.3 \mathrm{mM}$ ) of $\mathrm{PB}$ (solid) was added directly to the NMR tube with the equilibrium mixture of conjugates and the addition served as $t=0$ for the reduction. The one pot reductive amination experiments were performed by adding all reactants to the NMR tube at the same time, serving as $t=0.1 \mathrm{D}$ ${ }^{1} \mathrm{H}-\mathrm{NMR}$ spectra were recorded at desired time-points (600 MHz spectrometer) and the extent of conjugation/reduction was quantified by integration. The samples were held at room temperature (RT) between the measurements.

Chemical shift assignment for the equilibrium mixture obtained for the conjugation of PDHA to AA (2 equivalents PDHA, pH 4.0) was achieved using homo and heteronuclear NMR experiments recorded at the $800 \mathrm{MHz}$ spectrometer.

\subsection{Reduction of AA-ADH/PDHA conjugates, semi preparative scale}

AA (20.1 mM) and 10 equivalents of PDHA/ADH (201 mM) were dissolved in NaAc-buffer ( $500 \mathrm{mM}, \mathrm{pH} 4.0)$ to which 20 equivalents of PB $(420 \mathrm{mM})$ were added after $24 \mathrm{~h}$. The reactions were performed at room temperature for approx. 40 days. The reactions were terminated by dialysis ( $\mathrm{MWCO}=100-500 \mathrm{Da}$ ) against $0.05 \mathrm{M} \mathrm{NaCl}$ until the insoluble PB was dissolved, followed by several shifts of $\mathrm{MQ}$-water until the measured 
conductivity was $<2 \mu \mathrm{S} / \mathrm{cm}$ and subsequently freeze-dried. The mixture of products formed was separated by GFC (analytical scale), purified by dialysis and characterised by FIA-qTOF-MS. Selected products were additionally characterised by NMR spectroscopy (600 MHz spectrometer). Chemical shift assignment for the AA-PDHA secondary amine product was achieved using homo and heteronuclear NMR experiments recorded at the $800 \mathrm{MHz}$ spectrometer.

\subsection{Reduction at higher temperatures}

AA (20.1 mM) and 10 equivalents of PDHA/ADH (201 mM) were dissolved in NaAc-buffer (500 mM, pH 4.0) to which 20 or 40 equivalents $(402 \mathrm{mM}$ or $804 \mathrm{mM}$ ) of PB were added in 1 portion at $\mathrm{t}=0$ hours, or in portions after $\mathrm{t}=0,2,4,6$ and 8 hours. The reactions were performed in a water bath at 40 or $60^{\circ} \mathrm{C}$ for 8 or 24 hours, terminated by dialysis as described above and freeze-dried. The mixture of products formed was characterised by NMR spectroscopy (600 MHz spectrometer).

\section{Results and Discussion}

\subsection{Preparation of chitooligosaccharides}

Chitooligosaccharides of the type $D_{n} X A(X=A$ or $D)$ were obtained by enzymatic degradation of chitosan $\left(F_{A}\right.$ $=0.25$ ) using chitinase $B(C h i B)$ (Sørbotten et al., 2005). Oligomers of specific degree of polymerization (DP) were isolated using gel filtration chromatography (GFC) at $\mathrm{pH} 4.5$. The oligomers were mixtures of $\mathrm{D}_{n} \mathrm{DA}$ (51$66 \%$ ) and $D_{n} A A(34-49 \%)$. Subsequent GFC of oligomers with specific $D P$ at $p H 6.9$ separated $D_{n} D A$ from $D_{n} A A$ (e.g $D_{3} D A$ from $D_{3} A A$, Supporting Information, S2). However, complete baseline separation of the oligomers was not obtained e.g. resulting in residual $D_{2} D A(<15 \%)$ in the purified $D_{2} A A$ oligomer fraction.

\subsection{Conjugation of PDHA to AA}

The conjugation of PDHA to the disaccharide AA ( $N, N^{\prime}$-diacetylchitobiose, Figure $\left.3 a\right)$ was initially chosen as model system, since short oligosaccharides give relatively simple NMR spectra. Further, it allows for direct comparison to recently published data on conjugation of an ethoxyamine to mono- and disaccharides (Baudendistel et al., 2016). Compared to the protocol of Baudendistel et al. (2016) lower oligomer concentrations were used due to solubility limitations for oligomers of higher DPs. The concentration of dioxyamine was, on the other hand, increased to obtain higher reaction rates and to keep the formation of doubly substituted PDHA to a minimum.

The reaction with 2 equivalents of $\mathrm{PDHA}$ at $\mathrm{pH} 4.0$, was monitored by recording ${ }^{1} \mathrm{H}-\mathrm{NMR}$ spectra at regular intervals until equilibrium was reached (Figure $3 b$ ). The equilibrium mixture was studied in more detail by homo- and heteronuclear NMR correlation experiments (Supporting Information, S3) to assign key resonances. The course of the reaction was obtained by integration of the recorded spectra. The corresponding yields were calculated relative to the total intensity of $\mathrm{H} 1$ protons resulting from the reducing end A-unit (sum of unreacted oligomers and conjugates (oximes and $\mathrm{N}$-pyranosides), Figure $3 \mathrm{c}$ ). The ratio of acyclic $((E)$ - and $(Z)$-oximes) to cyclic ( $\beta-N$-pyranosides) conjugates was 3:1 at equilibrium (Table 1$)$, which is 
in good agreement with the conjugation of ethoxyamine to AA (Baudendistel et al., 2016), as was the absence of detectable amounts of furanosides and $\alpha-N$-pyranoside.

Two equivalents of PDHA resulted in a combined equilibrium yield of $88 \%$ conjugates and $12 \%$ unreacted oligomers. By treating the combined yield as a single product, the reaction was fitted to a pseudo first order kinetics model (Supporting Information, S4). The rate constants for the conjugation $\left(\mathrm{k}_{1}\right)$ and the reverse reaction $\left(k_{-1}\right)$ are included in Table 1 . The times to reach 50 and $90 \%$ of the combined yield at equilibrium $\left(t_{0.5}\right.$ and $t_{0.9}$ values) are further included as a more empirical approach to describe the reaction rates (Baudendistel et al., 2016). Increasing the PDHA concentration to 10 equivalents resulted in correspondingly higher reaction rates, but practically the same rate constants. It also resulted in almost full conversion (combined yield of $96 \%$ ) but did not significantly influence the ratio of acyclic to cyclic conjugates (Table 1).

The influence of $\mathrm{pH}$ on the combined yield and reaction kinetics with 2 equivalents of PDHA is shown in Figure $3 \mathrm{~d}$. Whereas the forward reaction rate $\left(\mathrm{k}_{1}\right)$ depended marginally on $\mathrm{pH}$, the reverse reaction rate $\left(\mathrm{k}_{-1}\right)$ was essentially proportional to $\left[\mathrm{H}^{+}\right]$, resulting in a decreasing yield with decreasing $\mathrm{pH}$ as also observed for other oxyamine conjugation reactions (Baudendistel et al., 2016). We partly attribute this to the contribution from acid lability of $\mathrm{N}$-pyranosides (Shinohara et al., 1996), which is consistent with the reduced fraction of cyclic conjugates at pH 3.0 (Table 1). It may be noted that the $\mathrm{pK}_{\mathrm{a}}$ of PDHA was found to be 4.2 (Supporting Information, S5). Hence, the weak pH dependency of $\mathrm{k}_{1}$ in the range investigated may be attributed to the opposing effects of the decrease in reactive (deprotonated) PDHA below $\mathrm{pK}_{\mathrm{a}}$, and the increasing rate of acid catalysis at lower $\mathrm{pH}$.

\subsection{Conjugation of ADH to AA}

Conjugation of the dihydrazide, $\mathrm{ADH}$ was studied using the same methods and conditions as described for the PDHA. The conjugation of ADH to AA deviated considerably from the reactions with PDHA (Figure 3e-h, Table 1). Firstly, only cyclic conjugates ( $\beta-N$-pyranoside and a minor unidentified $N$-glycoside product) were formed and no $(E)$ - and $(Z)$-hydrazones were detected. Secondly, two conformers of the $\beta$ - $N$-pyranoside, one major and one minor, were observed (Figure $3 e$ and f), which is attributed to the partial double bond character of the hydrazide (Bendiak, 1997). Thirdly, the reaction rate was two to five times lower than for the PDHA reactions. However, as also observed for PDHA, $\mathrm{k}_{1}$ was weakly dependent on $\mathrm{pH}$ (in the $\mathrm{pH}$ range 3.0-5.0), whereas the reverse rate constant $\left(\mathrm{k}_{-1}\right)$ increased with decreasing $\mathrm{pH}$, although not as pronounced as for PDHA (Figure 3h). As in the former case this may be partly attributed to acid hydrolysis of the $\mathrm{N}$-pyranosides (Shinohara et al., 1996). Overall, the combined yield increased with increasing $\mathrm{pH}$, but less pronounced than for PDHA. The differences are primarily attributed to the lower $\mathrm{pK}_{\mathrm{a}}$ of $\mathrm{ADH}$, which was found to be 3.1 (Supporting Information, S5).

High yields and short reaction times are desired for the preparation of conjugates. Hence, carrying out the reaction at $\mathrm{pH} 4.0$ and using a large excess of $\mathrm{PDHA}$ or $\mathrm{ADH}$ (10 equivalents or more) give the best compromise between yield and reaction time. Accordingly, all subsequent conjugation reactions were performed at $\mathrm{pH} 4.0$. 
a)

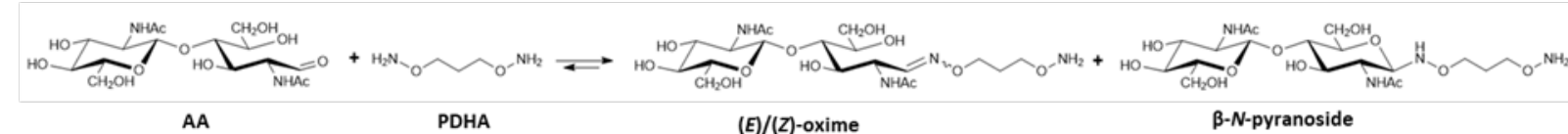

b)

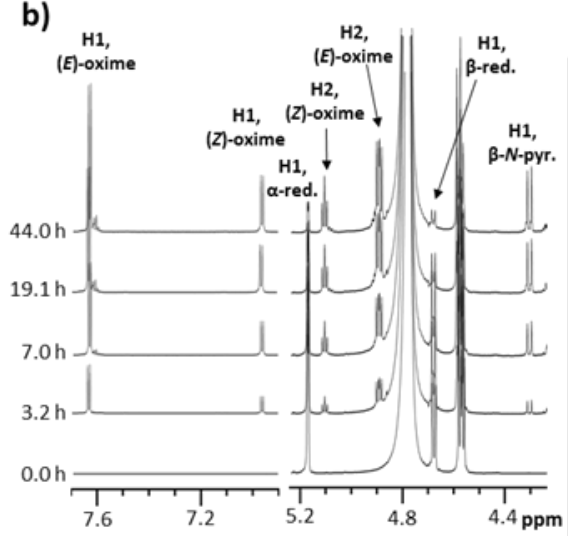

c)

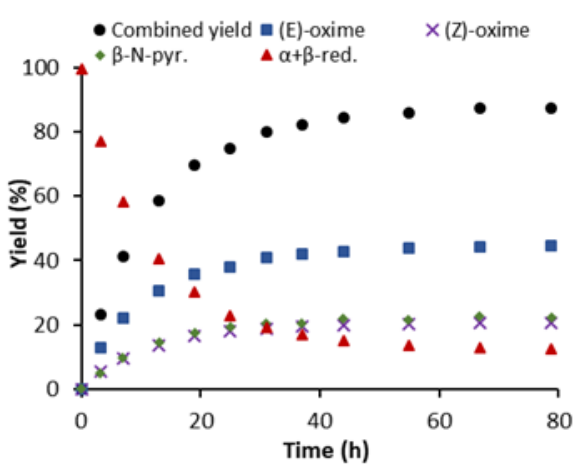

d)

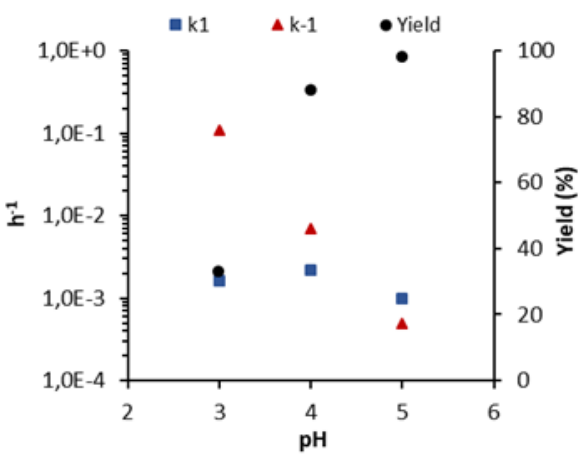

e)

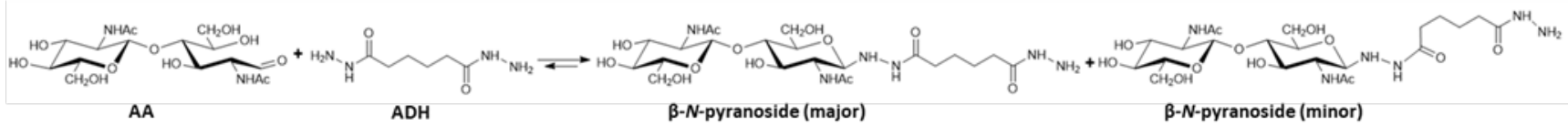

f)

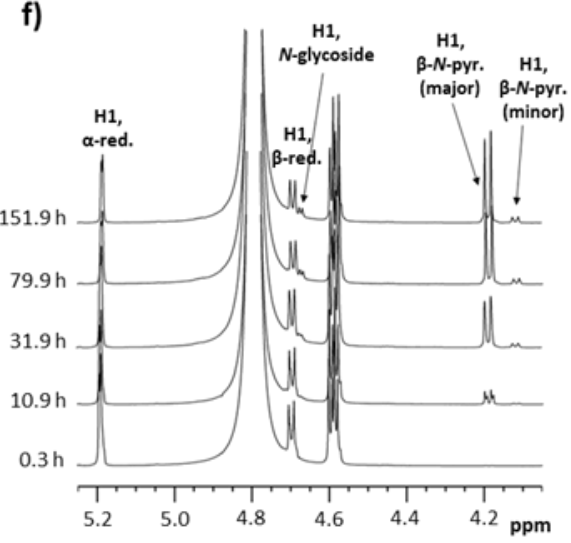

g)

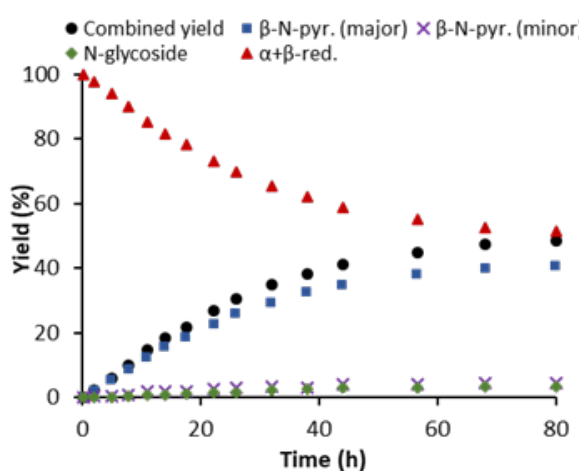

h)

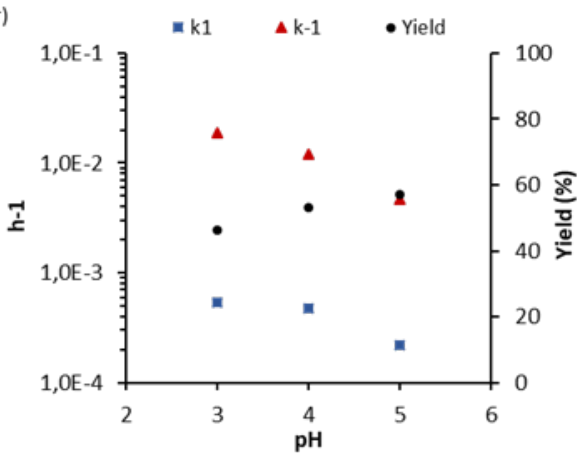

Figure 3. Reactions of $A A$ with $P D H A(a-d)$ and $A D H(e-h)$. a) and e) Conjugation of PDHA/ADH to $A A, \mathbf{b}$ ) and f) ${ }^{1} \mathrm{H}-\mathrm{NMR}$ spectra at defined time points for the conjugation reactions with 2 equivalents PDHA/ADH at $\mathrm{pH} 4.0, \mathbf{c}$ ) and $\mathbf{g}$ ) course of the reactions obtained from the integrals of spectra shown in b) and f), $\mathbf{d}$ ) and $\mathbf{h}$ ) influence of $\mathrm{pH}$ on combined yield and rate constants for the conjugation and the reverse reaction. Abbreviations: $\alpha$-red. and $\beta$-red.: $\alpha$ - and $\beta$-configurations of the unreacted oligomer, respectively. $\beta$ - $N$-pyr.: $\beta$ configuration of the $\mathrm{N}$-pyranoside.

Table 1. Reactions of AA with PDHA and ADH studied by time course NMR. ${ }^{1} \mathrm{H}-\mathrm{NMR}$ spectra of the equilibrium mixtures obtained for all the reactions are given in Supporting Information, S6.

\begin{tabular}{cccccccccc}
\hline Reaction & $\begin{array}{c}\text { PDHA/ADH } \\
\text { [equivalents] }\end{array}$ & $\mathbf{p H}$ & $\begin{array}{c}\mathbf{T} \\
{\left[{ }^{\circ} \mathrm{C}\right]}\end{array}$ & $\begin{array}{c}\text { Ratio } \\
\text { acycl.:cycl. }\end{array}$ & $\begin{array}{c}\mathbf{t}_{0.5} \\
{[\mathrm{~h}]}\end{array}$ & $\begin{array}{c}\mathbf{t}_{0.9} \\
{[\mathrm{~h}]}\end{array}$ & $\begin{array}{c}\mathbf{k}_{1} \\
{\left[\mathbf{h}^{-1}\right]}\end{array}$ & $\begin{array}{c}\mathbf{k}-1 \\
{\left[\mathbf{h}^{-1}\right]}\end{array}$ & $\begin{array}{c}\text { Combined } \\
\text { equilibrium yield } \\
{[\%]}\end{array}$ \\
\hline AA-PDHA & 2 & 3.0 & RT & $4.6: 1$ & 3.4 & 12.0 & $1.6 \times 10^{-3}$ & $1.1 \times 10^{-1}$ & 33 \\
& 2 & 4.0 & RT & $2.9: 1$ & 7.6 & 30.0 & $2.2 \times 10^{-3}$ & $7.0 \times 10^{-3}$ & 88 \\
& 2 & 5.0 & RT & $3.6: 1$ & 20.4 & 84.6 & $1.0 \times 10^{-3}$ & $5.0 \times 10^{-4}$ & 98 \\
& 4.0 & RT & $3.1: 1$ & 2.8 & 9.7 & $1.3 \times 10^{-3}$ & $9.0 \times 10^{-3}$ & 96
\end{tabular}




$\begin{array}{llllll}2 & 3.0 & \text { RT } & 1: 47.2 & 15.1 & 52.8 \\ 2 & 4.0 & \text { RT } & 1: 47.2 & 20.4 & 71.4 \\ 2 & 5.0 & \text { RT } & 1: 42.5 & 46.6 & 116.0 \\ 10 & 4.0 & \text { RT } & 1: 45.2 & 7.6 & 25.8\end{array}$

$\begin{array}{ll}5.4 \times 10^{-4} & 1.9 \times 10^{-2} \\ 4.7 \times 10^{-4} & 1.2 \times 10^{-2} \\ 2.2 \times 10^{-4} & 4.7 \times 10^{-3} \\ 4.0 \times 10^{-4} & 1.1 \times 10^{-2}\end{array}$

\subsection{Comparison to $D$ at the reducing end}

As a control experiment, 2 equivalents of PDHA or ADH were reacted with the disaccharide DD (chitobiose) under the same conditions as for $\mathrm{AA}$ ( $\mathrm{pH} 4.0, \mathrm{RT})$. DD showed poor reactivity compared to AA (Supporting information, S7), as demonstrated by very low yields (in the range 2-10\% after 72 hours), which is in agreement with the literature on other types of amines (Beaudoin et al., 2005; Guerry et al., 2013). Hence, D residues at the reducing end of chitooligosaccharides are very unfavourable for conjugations of this type.

\subsection{Conjugation of PDHA and ADH to longer chitooligosaccharides (DP 4-10)}

PDHA and $A D H$ were subsequently conjugated to $D_{n} X A$ oligomers (DP $=4-10, n=2-8$ ). The ${ }^{1} H-N M R$ spectra and the course of the reaction with $D_{2} X A(X=$ either $A$ or $D)$ and 2 equivalents of $A D H$ or PDHA, are shown in Supporting information, S8. For conjugation reactions with oligomers of DP $>4$, a mixture of $D_{n} X A(X=D$ or A) oligomers was used. Results from the conjugation reactions with $D_{n} X A$ oligomers are summarised in Table 2.

The reactions with longer chitooligosaccharides followed the same trends as observed for AA in terms of kinetics, yields and selectivity. Surprisingly, reactions with $D_{n} X A$ (Table 2) turned out to be slightly faster than with AA (Table 1). A similar dependency on DP has also reported for ethoxyamine, which conjugates faster to AA than A (monomer) (Baudendistel et al., 2016). For PDHA, the combined yield increased with higher DP of the chitooligosaccharides, whereas for ADH a slight decrease was observed.

The ratio of acyclic to cyclic conjugates increased for all reactions with the $D_{n} X A$ oligomers compared to $A A$ (Table 1) suggesting a higher stability of the oximes and hydrazones with increasing DP. This has also been observed when increasing the DP from one $(A)$ to two $(A A)$ shifting the ratio of acyclic to cyclic conjugates from 3.1:1 to $3.7: 1$ (Baudendistel et al., 2016)

Table 2. Reactions of $D_{n} X A$ oligomers with PDHA and ADH studied by time course NMR. ${ }^{1} H-N M R$ spectra of the equilibrium mixtures obtained for all the reactions are given in Supporting Information, S9.

\begin{tabular}{|c|c|c|c|c|c|c|c|c|c|}
\hline Reaction & $\begin{array}{l}\text { PDHA/ADH } \\
\text { [equivalents] }\end{array}$ & $\mathrm{pH}$ & $\begin{array}{c}\mathrm{T} \\
{\left[{ }^{\circ} \mathrm{C}\right]}\end{array}$ & $\begin{array}{c}\text { Ratio } \\
\text { acycl.:cycl. }\end{array}$ & $\begin{array}{l}\mathrm{t}_{0.5} \\
{[\mathrm{~h}]}\end{array}$ & $\begin{array}{l}\mathrm{t}_{0.9} \\
{[\mathrm{~h}]}\end{array}$ & $\begin{array}{c}k_{1} \\
{\left[h^{-1}\right]}\end{array}$ & $\begin{array}{l}k-1 \\
{\left[h^{-1}\right]}\end{array}$ & $\begin{array}{c}\text { Combined } \\
\text { equilibrium yield } \\
{[\%]}\end{array}$ \\
\hline $\mathrm{D}_{2} \mathrm{DA}-\mathrm{PDHA}$ & 2 & 4.0 & RT & $7.6: 1$ & 5.8 & 24.2 & $3.3 \times 10^{-3}$ & $1.5 \times 10^{-3}$ & 98 \\
\hline $\mathrm{D}_{8} \mathrm{XA}-\mathrm{PDHA}$ & 10 & 4.0 & RT & $5.4: 1$ & 1.6 & 5.4 & $2.2 \times 10^{-3}$ & $6.0 \times 10^{-3}$ & 98 \\
\hline $\mathrm{D}_{2} \mathrm{AA}-\mathrm{ADH}$ & 2 & 4.0 & RT & $1: 27.5$ & 19.9 & 69.2 & $4.3 \times 10^{-4}$ & $1.4 \times 10^{-2}$ & 48 \\
\hline $\mathrm{D}_{8} \mathrm{XA}-\mathrm{ADH}$ & 10 & 4.0 & RT & $1: 20.5$ & 5.8 & 19.7 & $5.2 \times 10^{-4}$ & $1.5 \times 10^{-2}$ & 86 \\
\hline
\end{tabular}

\subsection{Reduction of conjugates using $\alpha$-picoline borane (PB)}


Reduction of $\mathrm{D}_{2} \mathrm{AA}$-conjugates, prepared using 10 equivalents PDHA or $\mathrm{ADH}$ ( $\mathrm{pH} 4.0$ ), was studied by NMR, by adding solid $\alpha$-picoline borane (PB) directly to the NMR tube containing the oligosaccharide-PDHA/ADH equilibrium mixtures. PB has limited solubility under these conditions, and therefore, only 3 equivalents were used. The methylene protons of the secondary amines formed after reduction, gave proton resonances in the area 2.80 to $3.40 \mathrm{ppm}$, in agreement with literature data (Ridley et al., 1997), however partially overlapping with the $\mathrm{H} 2$ resonances of the D-units between 3.10 to $3.25 \mathrm{ppm}$ (Supporting information, S10). Results obtained after 2 and 14 days of reduction are given in Table 3. For both reactions the reduction was exceptionally slow, and a large fraction of unidentified products was detected. PB, both in its reduced $(r)$ and oxidized (o) form, has well defined proton resonances in the area 7.3 to $8.7 \mathrm{ppm}$, sufficiently separated from conjugate resonances in the same area, enabling the oxidation of the reducing agent to be monitored (Supporting information, S10). For the $\mathrm{D}_{2} \mathrm{AA}-\mathrm{PDHA}$ system $97 \%$ of the dissolved PB was oxidised after 14 days, whereas $33 \%$ of the dissolved PB was still in the reduced form in the $D_{2} A A-A D H$ system, indicating the slower reduction of the latter.

Table 3. Yields obtained at different time points during the reduction of conjugates, monitored by NMR. ${ }^{1} \mathrm{H}-\mathrm{NMR}$ spectra of the mixtures after 2 and 14 days are given in Supporting Information, S10.

\begin{tabular}{lccccccc}
\hline Reaction & $\begin{array}{c}\text { PDHA/ADH } \\
\text { [equivalents] }\end{array}$ & $\begin{array}{c}\text { PB } \\
\text { [equivalents] }\end{array}$ & $\begin{array}{c}\text { Time } \\
\text { [days] }\end{array}$ & $\begin{array}{c}\text { Reduced } \\
\text { conjugates } \\
{[\%]}\end{array}$ & $\begin{array}{c}\text { Non-reduced } \\
\text { conjugates } \\
\text { [\%] }\end{array}$ & $\begin{array}{c}\text { Unreacted } \\
\text { oligomers } \\
\text { [\%] }\end{array}$ & $\begin{array}{c}\text { Unidentified } \\
\text { products }\end{array}$ \\
\hline [\%]
\end{tabular}

[a] The yield of unidentified products was calculated as the difference between the formation of reduced (secondary amine) conjugates and the decrease in non-reduced conjugates and unreacted oligomers. Hence, yield of unidentified products $(\%)=$ theoretical yield $(100$ $\%$ ) - yield of reduced conjugates (\%) - yield of non-reduced conjugates (\%) - yield of unreacted oligomers (\%).

\subsection{Improvement of the reduction protocol}

Reduction was further explored using higher concentrations of PB and higher temperatures. As PB has limited solubility in aqueous buffer at room temperature, continuous monitoring of the reactions by NMR was impractical. Therefore, only the products obtained at the end of the reactions were studied. Poor mixing during the time course NMR experiments may have lowered the reduction rate and hence, regular mixing was applied, except at higher temperatures which increase the solubility of PB (see below). Optimisation of the reduction protocol was performed using the dimer, $\mathrm{AA}$, primarily to avoid the overlapping resonances from $\mathrm{H} 2$ of the D-units in the NMR-spectra.

First, AA-PDHA and AA-ADH conjugates, prepared using optimised conjugation conditions (10 equivalents PDHA/ADH, pH 4.0), were reduced using 20 equivalents $P B$. The reduction was performed for 40 days at room temperature, and the reaction mixtures were purified and fractionated by GFC. All fractions were characterised by mass spectroscopy and the main fractions were further analysed by ${ }^{1} \mathrm{H}-\mathrm{NMR}$ (Supporting Information, S11). Annotated chromatograms based on the MS and NMR characterisation are given in Figure 4a and $\mathrm{c}$. 
a)

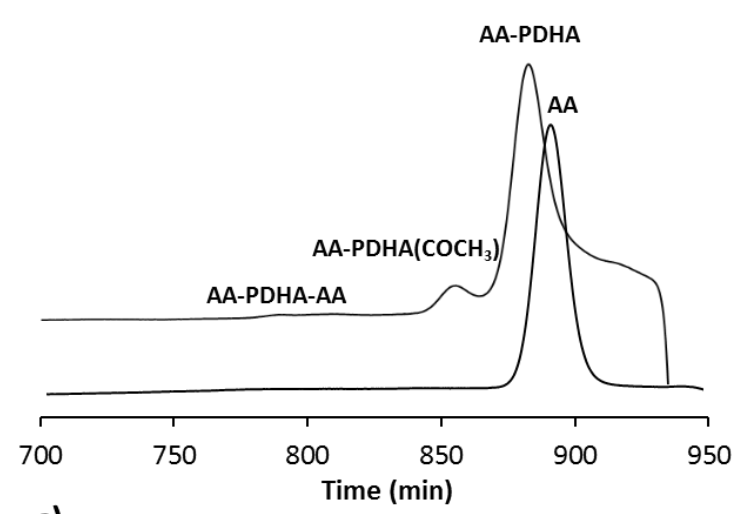

c)

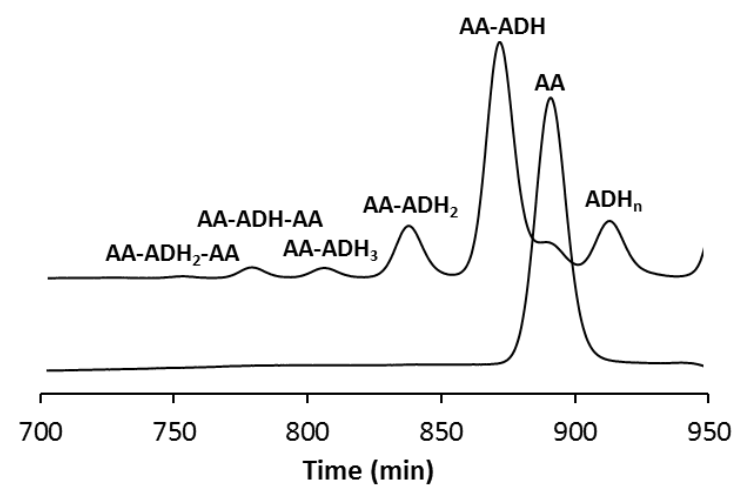

b)

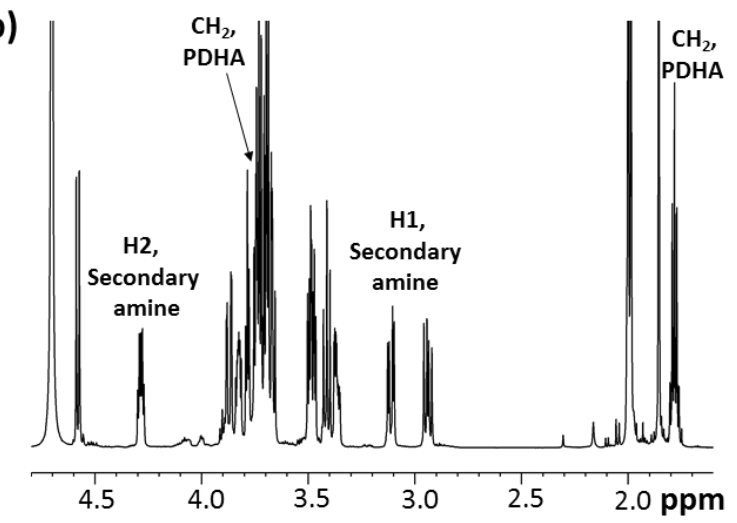

d)

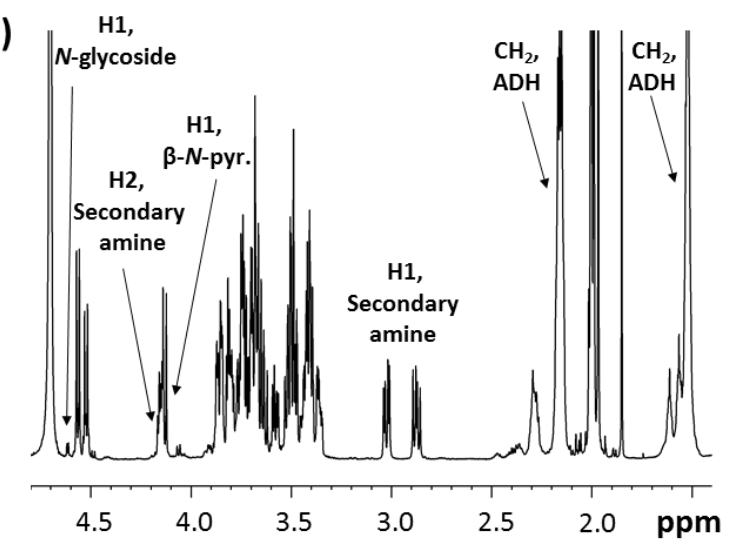

Figure 4. a) and c) GFC fractionation of the mixture of products formed in the conjugation of PDHA and ADH (10 equivalents) to AA with subsequent reduction for 40 days at $\mathrm{RT}$ using 20 equivalents $\mathrm{PB}$ at $\mathrm{pH} 4.0$, respectively. Fractionation of $\mathrm{AA}$ is included as a reference, $\mathbf{b}$ ) ${ }^{1} \mathrm{H}-\mathrm{NMR}$ spectrum of the main fraction from the AA-PDHA reaction, $\left.d\right)^{1} \mathrm{H}-\mathrm{NMR}$ spectrum of the main fraction from the AA-ADH reaction.

Reduction of AA-PDHA conjugates resulted in three fractions (Figure 4a) which based on MS and NMR were confirmed to be AA-PDHA (reduced to secondary amine), AA-PDHA-AA (doubly substituted and reduced), and a minor component with mass corresponding to acetylated AA-PDHA (reduced). The main fraction (reduced AA-PDHA), was further studied by homo- and heteronuclear NMR correlation experiments (Supporting Information, S12). Assignment of the main resonances are given in the ${ }^{1} \mathrm{H}-\mathrm{NMR}$ spectrum in Figure 4b. Integration confirmed an equimolar ratio of PDHA to AA. Resonances from $(E)$ - and (Z)-oximes and $\beta-N$-pyranoside were absent and no unreacted AA was detected. A small fraction of doubly substituted PDHA (AA-PDHA-AA) was formed despite the large excess (10 equivalents) of dioxyamine, suggesting an enhanced reactivity of the free oxyamine group following conjugation of PDHA to the first oligomer.

Reduction of AA-ADH conjugates resulted in a more complex mixture compared to PDHA (Figure 4c). ${ }^{1} \mathrm{H}$ NMR characterisation of the main fraction (Figure 4d), revealed a mixture of secondary amines (48\%) and non-reduced $\mathrm{N}$-glycosides (52\%). The apparent resistance to reduction of the latter is attributed to the slow (i.e. rate limiting) conversion to reducible hydrazones (Ramsay, Freeman, Grace, Redmond, \& MacLeod, 2001). In fact, $N$-glycosides were observed in all characterized fractions of the AA-ADH reaction. Detection of non-reduced, unreacted $A A$ (Figure 4c) confirm that PB selectively reduces Schiff bases under the given conditions, in addition to the slower conjugation of $\mathrm{ADH}$ compared to PDHA. 
As for PDHA, a fraction of doubly substituted ADH (AA-ADH-AA) was observed. Additionally, fractions containing conjugates with multiple dihydrazides $\left(\mathrm{ADH}_{\mathrm{n}}\right)$ were detected (Supporting Information, S11). To the best of our knowledge, this side reaction has not been described in the literature. These 'polymerised' dihydrazides also formed doubly substituted conjugates $\left(A A-A D H_{n}-A A\right)$. Conjugates containing $A_{D} H_{n}$ showed reduced solubility when re-dissolved in $\mathrm{D}_{2} \mathrm{O}$ for NMR characterisation. Hence, a purification step is required to obtain reduced conjugates without multiple dihydrazides.

Reduction was subsequently studied at 40 and $60^{\circ} \mathrm{C}$. Higher temperatures improves the solubility of PB, although at the expense of faster decomposition. AA was reacted with 10 equivalents of PDHA or ADH, and 20 equivalents of $\mathrm{PB}$ for 8 hours at $\mathrm{pH}$ 4.0. PB was added in one or several portions. Results and reaction conditions are given in Table 4. For AA-PDHA, the highest yield of reduced conjugates (49\%) could be obtained by combining 20 equivalents of $\mathrm{PB}$, added in four portions $(0,2,4$ and $6 \mathrm{~h})$, with reaction at $60^{\circ} \mathrm{C}$. For $\mathrm{ADH}$, the same protocol gave $26 \%$ reduced conjugates. By adding an additional portion of 20 equivalents PB after 8 hours and leaving the reaction mixture for a total of 24 hours at $60^{\circ} \mathrm{C}$, the yields were improved to $84 \%$ reduced AA-PDHA and $43 \%$ AA-ADH. The relative proportion of unidentified products decreased at higher temperatures, providing more optimised conditions for the reduction.

Table 4. Reaction conditions and yields obtained for one pot reductive amination at higher temperatures. ${ }^{1} \mathrm{H}-\mathrm{NMR}$ spectra of the reaction mixtures are given in Supporting Information, S13.

\begin{tabular}{|c|c|c|c|c|c|c|c|c|c|}
\hline Reaction & $\begin{array}{c}\mathrm{PB} \\
\text { [equivalents] }\end{array}$ & Portions & $\begin{array}{l}\text { Portions } \\
\text { added } \\
\text { [h] }\end{array}$ & $\begin{array}{c}\text { Total } \\
\text { rx. time } \\
\text { [h] }\end{array}$ & $\begin{array}{c}\mathrm{T} \\
{\left[{ }^{\circ} \mathrm{C}\right]}\end{array}$ & $\begin{array}{c}\text { Reduced } \\
\text { conjugates } \\
{[\%]}\end{array}$ & $\begin{array}{c}\text { Non- } \\
\text { reduced } \\
\text { conjugates } \\
{[\%]}\end{array}$ & $\begin{array}{c}\text { Unreacted } \\
\text { oligomers } \\
{[\%]}\end{array}$ & $\begin{array}{l}\text { Unidentified } \\
\text { products }^{[a]} \\
{[\%]}\end{array}$ \\
\hline \multirow[t]{5}{*}{ AA-PDHA } & 20 & 1 & 0 & 8 & $\overline{R T}$ & 11 & 87 & 1 & 1 \\
\hline & 20 & 1 & 0 & 8 & 40 & 24 & 54 & 2 & 20 \\
\hline & 20 & 1 & 0 & 8 & 60 & 40 & 37 & 1 & 22 \\
\hline & 20 & 4 & $0,2,4,6$ & 8 & 60 & 49 & 27 & 1 & 23 \\
\hline & 40 & 5 & $0,2,4,6,8$ & 24 & 60 & 84 & 13 & 0 & 3 \\
\hline \multirow[t]{5}{*}{$\overline{A A-A D H}$} & 20 & 1 & 0 & 8 & $\overline{R T}$ & 3 & 73 & 23 & 1 \\
\hline & 20 & 1 & 0 & 8 & 40 & 12 & 63 & 13 & 12 \\
\hline & 20 & 1 & 0 & 8 & 60 & 22 & 48 & 9 & 21 \\
\hline & 20 & 4 & $0,2,4,6$ & 8 & 60 & 26 & 39 & 7 & 28 \\
\hline & 40 & 5 & $0,2,4,6,8$ & 24 & 60 & 43 & 23 & 4 & 30 \\
\hline
\end{tabular}

[a] The yield of unidentified products was calculated as the difference between the formation of reduced conjugates and the decrease in non-reduced conjugates and unreacted oligomers. Hence, yield of unidentified products $(\%)=$ theoretical yield $(100 \%)-$ yield of reduced conjugates $(\%)$ - yield of non-reduced conjugates (\%) - yield of unreacted oligomers (\%).

Overall, the yield of reduced chitooligosaccharide conjugates could be improved by increasing the temperature and the concentration of PB (added in several portions to compensate for the fast decomposition at higher temperatures). Higher temperatures have also been shown to increase the fraction of reducible acyclic conjugates for oligosaccharide-oxyamine reactions (Baudendistel et al., 2016). 
The slower reduction of $\mathrm{ADH}$-conjugates compared to conjugates prepared with PDHA, both at room temperature and at higher temperatures, is attributed to the slow conversion of cyclic $\mathrm{N}$-glycosides to reducible acyclic conjugates combined with the large fraction of cyclic conjugates formed with ADH.

\subsection{Comparison to dextran oligomers}

To investigate whether the results obtained for the chitooligosaccharides were specific to the reducing end residue (GlcNAc), conjugation to a dextran oligomer (Dext $5_{5}$, DP 5) and the monomer (Glc, DP 1) was studied. Glc and Dext ${ }_{5}$ were reacted with PDHA or ADH (2 equivalents, $\mathrm{pH} 4.0$ ) and monitored by time course NMR as described above (Table 5). PDHA reacted two to three times faster than with the chitooligosaccharides, in excellent agreement with the Glc- and GIcNAc-ethoxyamine systems (Baudendistel et al., 2016). Moreover, the ratio of acyclic to cyclic conjugates was 2.6:1 for the monomer (Glc), which corresponds well to the results obtained with ethoxyamine under similar conditions (Baudendistel et al., 2016). As observed for the chitooligosaccharides, the ratio increased with increasing DP. Likewise, reaction rates increased slightly with increasing DP, whereas the yields slightly decreased.

In the case of $\mathrm{ADH}$, only $\mathrm{N}$-glycosides were formed when conjugated to Glc and Dext 5 , as also found for chitooligosaccharides. In contrast, ADH reacted 7-10 times faster than with the chitooligosaccharides. Hence, the nature of the residue at the reducing end (Glc versus GlcNAc) largely influences the conjugation rate, in agreement with other conjugation reactions (Baudendistel et al., 2016; Ramsay et al., 2001; Thygesen et al., 2010)

Table 5. Reactions of Dext ${ }_{n}$ oligomers with 2 equivalents PDHA or ADH studied by time course NMR. The course of the reaction for Dext $1_{1}$-PDHAADH is given in Supporting information, S14. ${ }^{1} \mathrm{H}-\mathrm{NMR}$ spectra of the equilibrium mixtures obtained for all the reactions are given in Supporting Information, S15.

\begin{tabular}{|c|c|c|c|c|c|c|c|c|c|}
\hline Reaction & $\begin{array}{l}\text { DP } \\
\text { [n] }\end{array}$ & $\mathrm{pH}$ & $\begin{array}{c}\mathrm{T} \\
{\left[{ }^{\circ} \mathrm{C}\right]}\end{array}$ & $\begin{array}{c}\text { Ratio } \\
\text { acycl.:cycl. }\end{array}$ & $\begin{array}{l}\mathrm{t}_{0.5} \\
{[\mathrm{~h}]}\end{array}$ & $\begin{array}{l}\mathrm{t}_{0.9} \\
{[\mathrm{~h}]}\end{array}$ & $\begin{array}{c}\mathbf{k}_{1} \\
{\left[\mathrm{~h}^{-1}\right]}\end{array}$ & $\begin{array}{c}k_{-1} \\
{\left[h^{-1}\right]}\end{array}$ & $\begin{array}{c}\text { Combined } \\
\text { equilibrium yield } \\
{[\%]}\end{array}$ \\
\hline \multirow{2}{*}{ Dext $_{n}-\mathrm{PDHA}$} & 1 & 4.0 & RT & 2.6:1 & 3.2 & 13 & $5.5 \times 10^{-3}$ & $1.2 \times 10^{-2}$ & 92 \\
\hline & 5 & 4.0 & RT & 4.3:1 & 2.2 & 8.6 & $8.0 \times 10^{-3}$ & $2.0 \times 10^{-2}$ & 90 \\
\hline \multirow{2}{*}{ Dext $_{n}-$ ADH } & 1 & 4.0 & RT & $1: 64$ & 3.0 & 10.4 & $2.9 \times 10^{-3}$ & $9.0 \times 10^{-2}$ & 49 \\
\hline & 5 & 4.0 & RT & 1:41 & 1.9 & 6.4 & $3.5 \times 10^{-3}$ & $1.9 \times 10^{-1}$ & 38 \\
\hline
\end{tabular}

We further monitored the one pot reductive amination of Dext 4 with 2 equivalents of PDHA or ADH in the presence of $\mathrm{PB}$ ( 3 equivalents) and compared the results with the reaction of $\mathrm{AA}$ with PDHA or ADH under the same conditions ( $\mathrm{RT}, \mathrm{pH}$ 4.0). The spectra obtained after 2 and 5 days for all reactions are given in Supporting information, S16. After 5 days, $70 \%$ reduced Dext $t_{4}$-PDHA and $61 \%$ Dext $_{4}-\mathrm{ADH}$ were formed, as opposed to only $9 \%$ and $6 \%$ reduced AA-PDHA and AA-ADH, respectively. In contrast to the AA reactions, where a large fraction of unidentified products was formed, all products could be accounted for in the Dext ${ }_{4}$ reactions (Supporting information, S16). We therefore attribute the slow and complex reduction of the chitooligosaccharide-based conjugates to the nature of the reducing end, i.e. the GIcNAc residue.

\section{Conclusions}


In this study we have presented a systematic investigation of aqueous, aniline-free conjugation of the bifunctional linkers PDHA and ADH to the reducing end of chitooligosaccharides ranging from DP 2 to DP 10, both without and with reduction using a-picoline borane. The oligomers were of the type $D_{n} X A$ obtained by enzymatic degradation of chitosan and are therefore relevant in a biorefinery context. As expected from literature, reactions with PDHA resulted in a mixture of acyclic $(E)$ - and $(Z)$-oximes and cyclic $\beta-N$ pyranosides, whereas ADH yielded only cyclic $\mathrm{N}$-glycosides. The acyclic/cyclic ratio of conjugates, combined yield, and reaction kinetics depended in both cases on $\mathrm{pH}$. In total, highest reaction rates were obtained at $\mathrm{pH}$ 3.0, whereas highest yields were obtained at $\mathrm{pH}$ 5.0. Carrying out the reaction at $\mathrm{pH} 4.0$ therefore gives a good compromise between reaction time and yield. The influence of chain length is of utmost importance for general use of the methodology. We found that the rate of conjugation was essentially independent of DP within the range investigated.

The conjugation of PDHA and ADH to dextran oligomers was included for comparison. In general, both PDHA and $\mathrm{ADH}$ conjugated faster to dextran than to the chitooligosaccharides and hence, the nature of the reducing end residue plays an important role for the outcome of the conjugation. In this perspective, it may be noted that oligomers terminating in $D$ residues were confirmed to be less reactive than those having $A$ at the reducing ends. Hence, the $D_{n} X A$ oligomers have an additional advantage over pure $D_{n}$ oligomers.

All protocols seem to give a small amount of disubstituted species, e.g. AA-PDHA-AA (Fig. 4a). This can in principle be tolerated in the subsequent coupling because they will be unreactive. They may alternatively be removed by chromatography as shown in the same figure. Further, detectable (but small) amounts of oligomers with multiple $\mathrm{ADH}$, i.e. $\mathrm{AA}-\mathrm{ADH}_{2}$ were detected (Fig. 4c). They may, as shown here, still react to form e.g. $A A-A D H_{2}-A A$, indicating the terminal $A D H$ remains reactive. If undesired, they may also be removed after the activation step, at least for short oligosaccharides.

Although a-picoline borane has proven useful in reductive amination with carbohydrates, we found that chitooligosaccharide-based PDHA- and ADH-conjugates were very slowly reduced at room temperature. However, a protocol using higher temperatures improved the reduction significantly with acceptable yields after 24 hours, comparable to aniline- and cyanoborohydride-based protocols (Guerry et al., 2013). We attribute the slow reduction to the nature of the reducing end residue, as dextran-based conjugates were reduced with a much higher rate under the same conditions. The slower reduction of ADH conjugates for both types of oligomers is attributed to the large fraction of non-reducible cyclic conjugates. In general, the slow reduction of the chitooligosaccharide conjugates is considered unsuitable for typical labelling applications in biochemistry but may still be more acceptable in biomaterials preparation.

In summary, the reaction of enzymatically produced chitooligosaccharides with PDHA and ADH, without or with reduction using a-picoline borane has been studied in detail. The outcome is protocols and kinetic constants for preparing both PDHA- and ADH-chitooligosaccharide conjugates. The protocols avoid toxic reagents such as sodium cyanoborohydride and aniline, and retains the inherent properties of the oligosaccharides. The PDHA- or ADH-activated $D_{n} X A$ chitooligosaccharide conjugates may be effectively linked to aldehyde-containing particles, surfaces or macromolecules e.g. other oligosaccharides for the preparation of block polysaccharides. 


\section{Acknowledgements}

This work was supported by a grant from the Norwegian University of Science and Technology to I.V. Mo It was further partially supported by grant 6167 from VISTA (a basic research program in collaboration between The Norwegian Academy of Science and Letters, and Statoil), and grants 268490, 226244 and 221576 from the Research Council of Norway. Vincent G. H. Eijsink is thanked for kindly providing ChiB. Odin Weberg Haarberg is thanked for running the conjugation experiments with dextran during his master work. Bård Helge Hoff and Hallvard Svendsen are thanked for useful discussions. Kåre Kristiansen is thanked for running the MS analysis and Ann-Sissel T. Ulset is thanked for technical assistance.

\section{References}

Allan, G. G., \& Peyron, M. (1995). Molecular-Weight Manipulation of Chitosan .1. Kinetics of Depolymerization by Nitrous-Acid. Carbohydrate Research, 277(2), 257-272.

Baudendistel, O. R., Wieland, D. E., Schmidt, M. S., \& Wittmann, V. (2016). Real-Time NMR Studies of Oxyamine Ligations of Reducing Carbohydrates under Equilibrium Conditions. Chemistry - A European Journal, 22(48), 17359-17365.

Beaudoin, M.-E., Gauthier, J., Boucher, I., \& Waldron, K. C. (2005). Capillary electrophoresis separation of a mixture of chitin and chitosan oligosaccharides derivatized using a modified fluorophore conjugation procedure. Journal of Separation Science, 28(12), 1390-1398.

Bendiak, B. (1997). Preparation, conformation, and mild hydrolysis of 1-glycosyl-2-acetylhydrazines of the hexoses, pentoses, 2-acetamido-2-deoxyhexoses, and fucose. Carbohydrate Research, 304(1), 85-90.

Breitenbach, B. B., Schmid, I., \& Wich, P. R. (2017). Amphiphilic Polysaccharide Block Copolymers for pHResponsive Micellar Nanoparticles. Biomacromolecules, 18(11), 3844-3845.

Cosenza, V. A., Navarro, D. A., \& Stortz, C. A. (2011). Usage of $\alpha$-picoline borane for the reductive amination of carbohydrates. Arkivoc, 12, 182-194.

Dalheim, M. Ø., Vanacker, J., Najmi, M. A., Aachmann, F. L., Strand, B. L., \& Christensen, B. E. (2016). Efficient functionalization of alginate biomaterials. Biomaterials, 80, 146-156.

Dirksen, A., \& Dawson, P. E. (2008). Rapid oxime and hydrazone ligations with aromatic aldehydes for biomolecular labeling. Bioconjugate chemistry, 19(12), 2543-2548.

Dirksen, A., Hackeng, T. M., \& Dawson, P. E. (2006). Nucleophilic catalysis of oxime ligation. Angewandte Chemie-International Edition, 45(45), 7581-7584.

Fang, J., Qin, G., Ma, J., \& She, Y.-M. (2015). Quantification of plant cell wall monosaccharides by reversedphase liquid chromatography with 2-aminobenzamide pre-column derivatization and a non-toxic reducing reagent 2-picoline borane. Journal of Chromatography A, 1414, 122-128.

Fina, N. J., \& Edwards, J. O. (1973). The alpha effect. A review. International Journal of Chemical Kinetics, 5(1), 1-26.

Guerry, A., Bernard, J., Samain, E., Fleury, E., Cottaz, S., \& Halila, S. (2013). Aniline-Catalyzed Reductive Amination as a Powerful Method for the Preparation of Reducing End- "Clickable" Chitooligosaccharides. Bioconjugate chemistry, 24(4), 544-549.

Hermanson, G. T. (2008). Bioconjugate Techniques. (2nd ed.). Boston: Academic Press.

Kalia, J., \& Raines, R. T. (2008). Hydrolytic Stability of Hydrazones and Oximes. Angewandte Chemie, 120(39), 7633-7636.

Krȩżel, A., \& Bal, W. (2004). A formula for correlating pKa values determined in D2O and H2O. Journal of Inorganic Biochemistry, 98(1), 161-166.

Kwase, Y. A., Cochran, M., \& Nitz, M. (2013). Protecting-Group-Free Glycoconjugate Synthesis: Hydrazide and Oxyamine Derivatives in N-Glycoside Formation. In D. B. Werz \& S. Vidal (Eds.), Modern synthetic 
methods in carbohydrate chemistry: From monosaccharides to complex glycoconjugates (pp. 67-96): Wiley

Kölmel, D. K., \& Kool, E. T. (2017). Oximes and Hydrazones in Bioconjugation: Mechanism and Catalysis. Chemical Reviews, 117(15), 10358-10376.

Lee, M.-r., \& Shin, I. (2005). Facile Preparation of Carbohydrate Microarrays by Site-Specific, Covalent Immobilization of Unmodified Carbohydrates on Hydrazide-Coated Glass Slides. Organic Letters, 7(19), 4269-4272.

Mellegård, H., Strand, S. P., Christensen, B. E., Granum, P. E., \& Hardy, S. P. (2011). Antibacterial activity of chemically defined chitosans: Influence of molecular weight, degree of acetylation and test organism. International Journal of Food Microbiology, 148(1), 48-54.

Moussa, A., Crepet, A., Ladaviere, C., \& Trombotto, S. (2019). Reducing-end "clickable" functionalizations of chitosan oligomers for the synthesis of chitosan-based diblock copolymers. Carbohydrate Polymers, 219, 387-394.

Novoa-Carballal, R., \& Müller, A. H. E. (2012). Synthesis of polysaccharide-b-PEG block copolymers by oxime click. Chemical Communications, 48(31), 3781-3783.

Pickenhahn, V. D., Darras, V., Dziopa, F., Biniecki, K., De Crescenzo, G., Lavertu, M., \& Buschmann, M. D. (2015). Regioselective thioacetylation of chitosan end-groups for nanoparticle gene delivery systems. Chemical Science, 6(8), 4650-4664.

Ramsay, S. L., Freeman, C., Grace, P. B., Redmond, J. W., \& MacLeod, J. K. (2001). Mild tagging procedures for the structural analysis of glycans. Carbohydrate Research, 333(1), 59-71.

Ridley, B. L., Spiro, M. D., Glushka, J., Albersheim, P., Darvill, A., \& Mohnen, D. (1997). A method for biotin labeling of biologically active oligogalacturonides using a chemically stable hydrazide linkage. Analytical Biochemistry, 249(1), 10-19.

Rosselgong, J., Chemin, M., Almada, C. C., Hemery, G., Guigner, J.-M., Chollet, G., . . Cramail, H. (2019). Synthesis and Self-Assembly of Xylan-Based Amphiphiles: From Bio-Based Vesicles to Antifungal Properties. Biomacromolecules, 20(1), 118-129.

Ruhaak, L. R., Steenvoorden, E., Koeleman, C. A. M., Deelder, A. M., \& Wuhrer, M. (2010). 2-Picoline-borane: A non-toxic reducing agent for oligosaccharide labeling by reductive amination. PROTEOMICS, 10(12), 2330-2336.

Ruhaak, L. R., Zauner, G., Huhn, C., Bruggink, C., Deelder, A. M., \& Wuhrer, M. (2010). Glycan labeling strategies and their use in identification and quantification. Analytical and Bioanalytical Chemistry, 397(8), 3457-3481.

Schatz, C., \& Lecommandoux, S. (2010). Polysaccharide-Containing Block Copolymers: Synthesis, Properties and Applications of an Emerging Family of Glycoconjugates. Macromolecular Rapid Communications, 31(19), 1664-1684.

Shinohara, Y., Sota, H., Gotoh, M., Hasebe, M., Tosu, M., Nakao, J., ... Shiga, M. (1996). Bifunctional Labeling Reagent for Oligosaccharides To Incorporate Both Chromophore and Biotin Groups. Analytical Chemistry, 68(15), 2573-2579.

Strand, S. P., Danielsen, S., Christensen, B. E., \& Vårum, K. M. (2005). Influence of Chitosan Structure on the Formation and Stability of DNA-Chitosan Polyelectrolyte Complexes. Biomacromolecules, 6(6), 33573366.

Sørbotten, A., Horn, S. J., Eijsink, V. G. H., \& Vårum, K. M. (2005). Degradation of chitosans with chitinase B from Serratia marcescens. The FEBS Journal, 272(2), 538-549.

Thygesen, M. B., Munch, H., Sauer, J., Cló, E., Jørgensen, M. R., Hindsgaul, O., \& Jensen, K. J. (2010). Nucleophilic Catalysis of Carbohydrate Oxime Formation by Anilines. The Journal of Organic Chemistry, 75(5), 1752-1755.

Tømmeraas, K., Vårum, K. M., Christensen, B. E., \& Smidsrød, O. (2001). Preparation and characterisation of oligosaccharides produced by nitrous acid depolymerisation of chitosans. Carbohydrate Research, 333(2), 137-144.

Unterieser, I., \& Mischnick, P. (2011). Labeling of oligosaccharides for quantitative mass spectrometry. Carbohydrate Research, 346(1), 68-75. 
Vårum, K. M., Antohonsen, M. W., Grasdalen, H., \& Smidsrød, O. (1991). Determination of the degree of Nacetylation and the distribution of $\mathrm{N}$-acetyl groups in partially $\mathrm{N}$-deacetylated chitins (chitosans) by high-field n.m.r. spectroscopy. Carbohydrate Research, 211(1), 17-23. 


\section{Supporting Information}

\section{Activation of enzymatically produced chitooligosaccharides by dioxyamines and dihydrazides}

Ingrid Vikøren Mo, Yiming Feng, Marianne Øksnes Dalheim, Amalie Solberg, Finn L. Aachmann, Christophe Schatz, and Bjørn E. Christensen

Contents

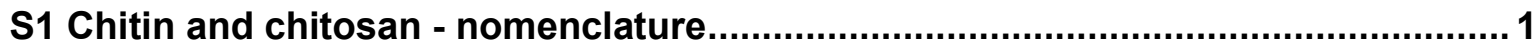

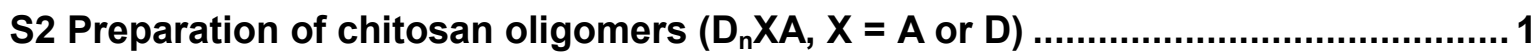

S3 2D Characterisation of the equilibrium mixture for the conjugation of PDHA to AA

S4 Pseudo first order kinetics modelling of the conjugation reactions ...................... 4

S5 $\mathrm{pH}$ titration by ${ }^{1} \mathrm{H}-\mathrm{NMR}$ to determine the $\mathrm{pK}_{\mathrm{a}}$ of PDHA and ADH .......................... 5

S6 ${ }^{1} \mathrm{H}-\mathrm{NMR}$ characterisation of the equilibrium mixtures for the conjugation

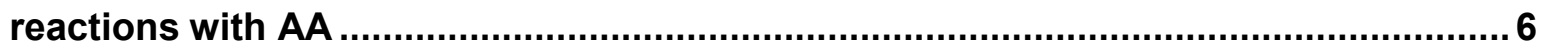

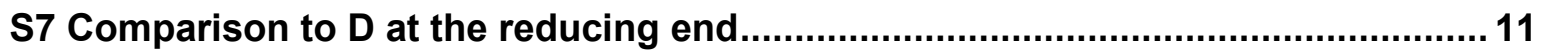

S8 Conjugation of PDHA and ADH to $D_{2} X A$ studied by time course NMR................11

S9 ${ }^{1} \mathrm{H}-\mathrm{NMR}$ characterisation of the equilibrium mixtures for the conjugation reactions with $D_{n} X A$ oligomers.

S10 ${ }^{1} \mathrm{H}-\mathrm{NMR}$ characterisation of the reaction mixtures for the $\mathrm{D}_{\mathrm{n}} \mathrm{XA}-\mathrm{PDHA} / \mathrm{ADH}$ conjugates after 2 and 14 days of reduction

S11 ${ }^{1} \mathrm{H}-\mathrm{NMR}$ and MS characterisation of products formed in the reduction of AAPDHA and AA-ADH conjugates using PB

S12 2D NMR characterisation of the reduced AA-PDHA conjugate.

S13 ${ }^{1} \mathrm{H}$-NMR characterisation of the reaction mixtures for the reduction of conjugates at higher temperatures

S14 Conjugation of PDHA and ADH to $\operatorname{Dext}_{1}$ (GIc) studied by time course NMR ..... 27

$\mathrm{S} 15{ }^{1} \mathrm{H}-\mathrm{NMR}$ characterisation of the equilibrium mixtures for the conjugation reactions with Dext $_{n}$ oligomers

S16 ${ }^{1} \mathrm{H}$-NMR characterisation of the reaction mixtures for the one pot reductive amination reaction of $A A$ and Dext $_{4}$ with PDHA or ADH after 2 and 5 days. 30

S16 References. 


\section{S1 Chitin and chitosan - nomenclature}

In order to obtain unique but simple representations of monomer sequences, the chitin monomer, $\mathrm{N}$ acetyl-D-glucosamine (GlcNAc), and its de- $N$-acetylated counterpart, D-glucosamine (GlcN) are abbreviated with the single letters $A$ and $D$, respectively. Accordingly, the dimer $N, N^{\prime}$-diacetyl chitobiose is abbreviated AA and chitobiose is abbreviated DD. The monomers are linked by $\beta-1,4-$ linkages in both chitin and chitosan. The composition of chitosans is commonly described by the fraction of acetylated units $\left(F_{A}\right)$, hence, the fraction of $A$ residues in the polysaccharide. $F_{A}$ is defined as

$$
F_{A}=n_{A} /\left(n_{A}+n_{D}\right)
$$

where $n_{A}$ is the number of A-units and $n_{D}$ is the number of D-units in the chitosan. Another commonly used parameter for describing chitosan is the degree of de- $N$-acetylation, describing the fraction of $A$ units in the chitosan related to $F_{A}$ by the equation

$$
\text { Degree of de }-N \text {-acetylation }=\left(1-F_{A}\right) \times 100 \%
$$

\section{S2 Preparation of chitosan oligomers $\left(D_{n} X A, X=A\right.$ or $\left.D\right)$}

Water soluble chitosan with $F_{A}=0.25$ was prepared by partial de- $N$-acetylation of chitin. The chitosan was further subjected to enzymatic degradation with chitinase B (ChiB) from Serratia marcescens. ChiB belongs to the $\mathrm{GH} 18$ family and has a specified cleavage site for $\mathrm{N}$-acetyl glucosamine linkages (van Aalten et al., 2001). The enzyme works from the non-reducing end and cleaves the polymer after one or two A-units (preferably two A units in the -1 and -2 subsites, but will also cleave the polymer when a $D$-unit is present in the -2 subsite, however with a slower rate). Hence, depending on the $F_{A}$ of the chitosan and the conditions, the enzymatic degradation can exclusively provide chitooligosaccharides terminating in one or two A residues at the reducing end, but which otherwise contain uninterrupted (homogenous) sequences of $D$ residues $\left(D_{n}\right)$ (Sørbotten, Horn, Eijsink, \& Vårum, 2005). Oligomers with specific $D P\left(D_{n} X A, n=2-8\right)$ were fractionated by gel filtration chromatography (GFC) using $0.15 \mathrm{M}$ ammonium acetate (AmAc) buffer, pH 4.5 (Figure S1a). Fractions were purified by dialysis and freeze-dried and the DP of the oligomers was determined by ${ }^{1} \mathrm{H}-\mathrm{NMR}$ spectroscopy. Individual fractions were subsequently re-chromatographed using $0.1 \mathrm{M} \mathrm{AmAc}$ buffer adjusted to $\mathrm{pH}$ 6.9. This step separated $\mathrm{D}_{n} A A$ from $\mathrm{D}_{n} \mathrm{DA}$ (verified by NMR), although baseline separation only was obtained for $n=2-3$ (Figure S1b).

a)

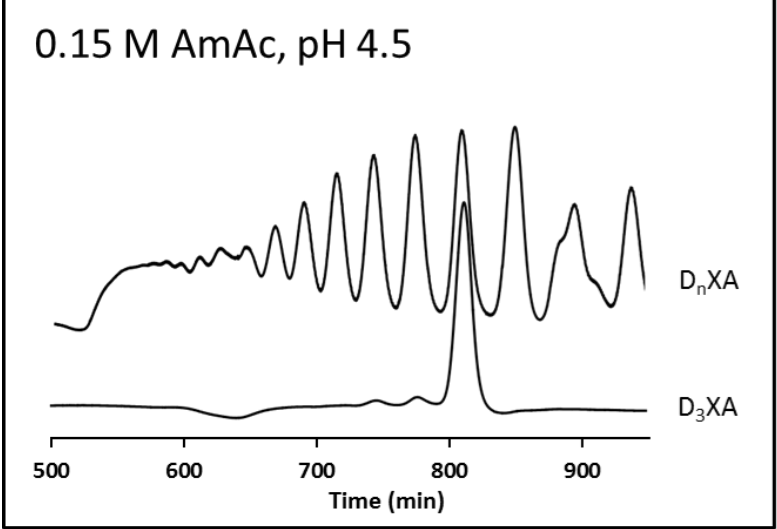

b)

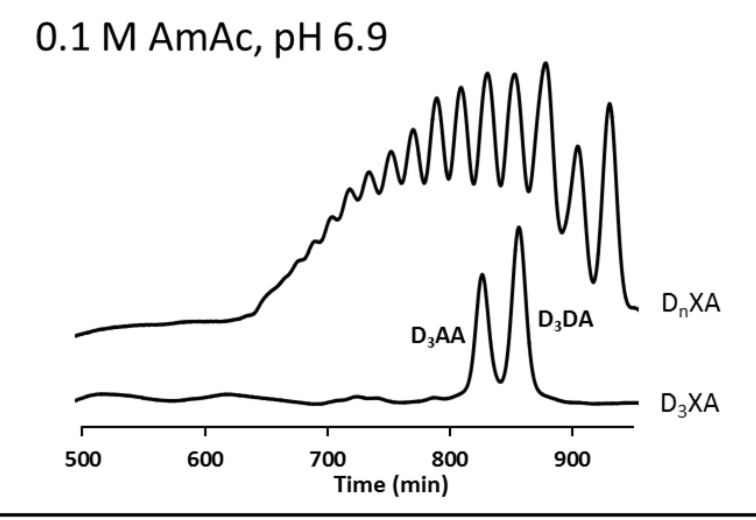

Figure S1: a) GFC fractionation of the mixture of $D_{n} X A$ oligomers obtained by enzymatic degradation of chitosan $\left(F_{A}=0.25\right)$ using $\mathrm{ChiB}$ in $0.15 \mathrm{M} \mathrm{AmAc}$ at $\mathrm{pH}$ 4.5. Fractionation of isolated $D_{3} X A$ is included to show the DP distribution. b) GFC fractionation of the mixture of $D_{n} X A$ oligomers in $0.1 \mathrm{M} \mathrm{AmAc}$ at $\mathrm{pH}$ 6.9. Fractionation of $\mathrm{D}_{3} \mathrm{XA}$ is included to demonstrate the ability to separate oligomers with different chemical composition. 
Isolated chitooligosaccharides were characterized by ${ }^{1} \mathrm{H}-\mathrm{NMR}$. The spectrum obtained for the isolated $D_{2} A A$ oligomers were assigned according to literature (Figure S2) (Sugiyama et al., 2001). Isolated $\mathrm{D}_{2} \mathrm{AA}$ contained approximately $15 \% \mathrm{D}_{2} \mathrm{DA}$, evident in the ${ }^{1} \mathrm{H}$ NMR-spectrum by side peaks for the reducing end (r.e.) resonances ( $\mathrm{H} 1, \mathrm{~A}$ r.e. in $\alpha$ - and $\beta$-configuration). The middle $\mathrm{D}$ - and $\mathrm{A}$ units are assigned $m$, whereas the non-reducing end $D$-unit is assigned n.r.e. The chemical shifts of the resonances resulting from the $\mathrm{H} 2$ of the D-units ( $m$ and n.r.e.) are very $\mathrm{pH}$ sensitive and increasing the $\mathrm{pH}$ above the $\mathrm{pK}_{\mathrm{a}}$ of the amino group (approx. 6.5) shifts the resonances upfield to 2.5$2.7 \mathrm{ppm}$.

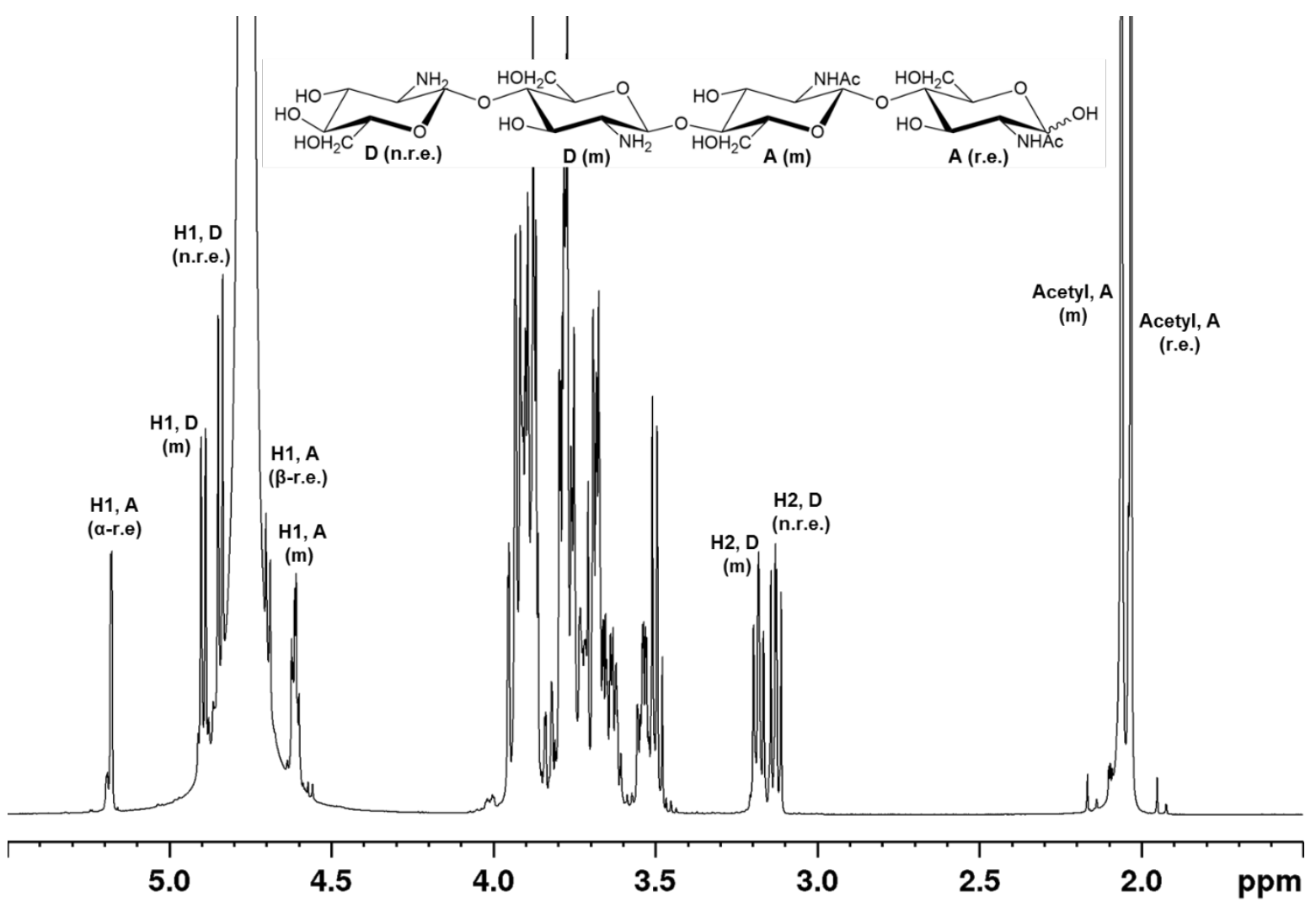

Figure S2: ${ }^{1} \mathrm{H}-\mathrm{NMR}$ spectrum of isolated $\mathrm{D}_{2} \mathrm{AA}$ at $\mathrm{pH} 4.0,298 \mathrm{~K}$.

\section{S3 2D Characterisation of the equilibrium mixture for the conjugation of PDHA to AA}

The dimer AA ( $N, N^{\prime}$-diacetyl chitobiose, $20.1 \mathrm{mM}$ ) was reacted with 2 equivalents of PDHA under standard reaction conditions ( $500 \mathrm{mM}$ deuterated $\mathrm{NaAc}$-buffer $\mathrm{pH} 4.0$, room temperature).

Resonances from conjugates and reactants at equilibrium ( $>48 \mathrm{~h}$ ) were assigned by homo and hetero nuclear NMR correlation experiments. The NMR analysis was carried out at the $800 \mathrm{MHz}$ spectrometer in a $3 \mathrm{~mm}$ NMR tube. Resonances were assigned by starting at the anomeric proton resonance and then following the proton-proton connectivity using TOCSY, DQF-COSY, and ${ }^{13} \mathrm{C}$ HSQC $-\left[{ }^{1} \mathrm{H},{ }^{1} \mathrm{H}\right]$ TOCSY spectra. The carbon chemical shifts were obtained from ${ }^{13} \mathrm{C} \mathrm{HSQC} .{ }^{13} \mathrm{C} \mathrm{HMBC}$ was used for connecting the spin systems via long range connections. The following designations are used in the spectra displayed in Figure S3 and S4: $(E)$ and $(Z)((E) /(Z)$-configuration of the oxime), $\beta$ $N$-pyranoside ( $\beta$-configuration of the $N$-pyranoside), $\alpha$ - and $\beta$-red. (anomeric protons of the reducing A-unit), NR (anomeric proton of the non-reducing A-unit), L\# (methylene protons from conjugated dioxyamine (PDHA)), L' (methylene protons from unreacted PDHA), Ac ( $\mathrm{N}$-acetyl groups of the Aunits). $\mathrm{H} \#$ refers to the proton attached to the ring carbon number $(\mathrm{C \# )}$ for the sugar units. 


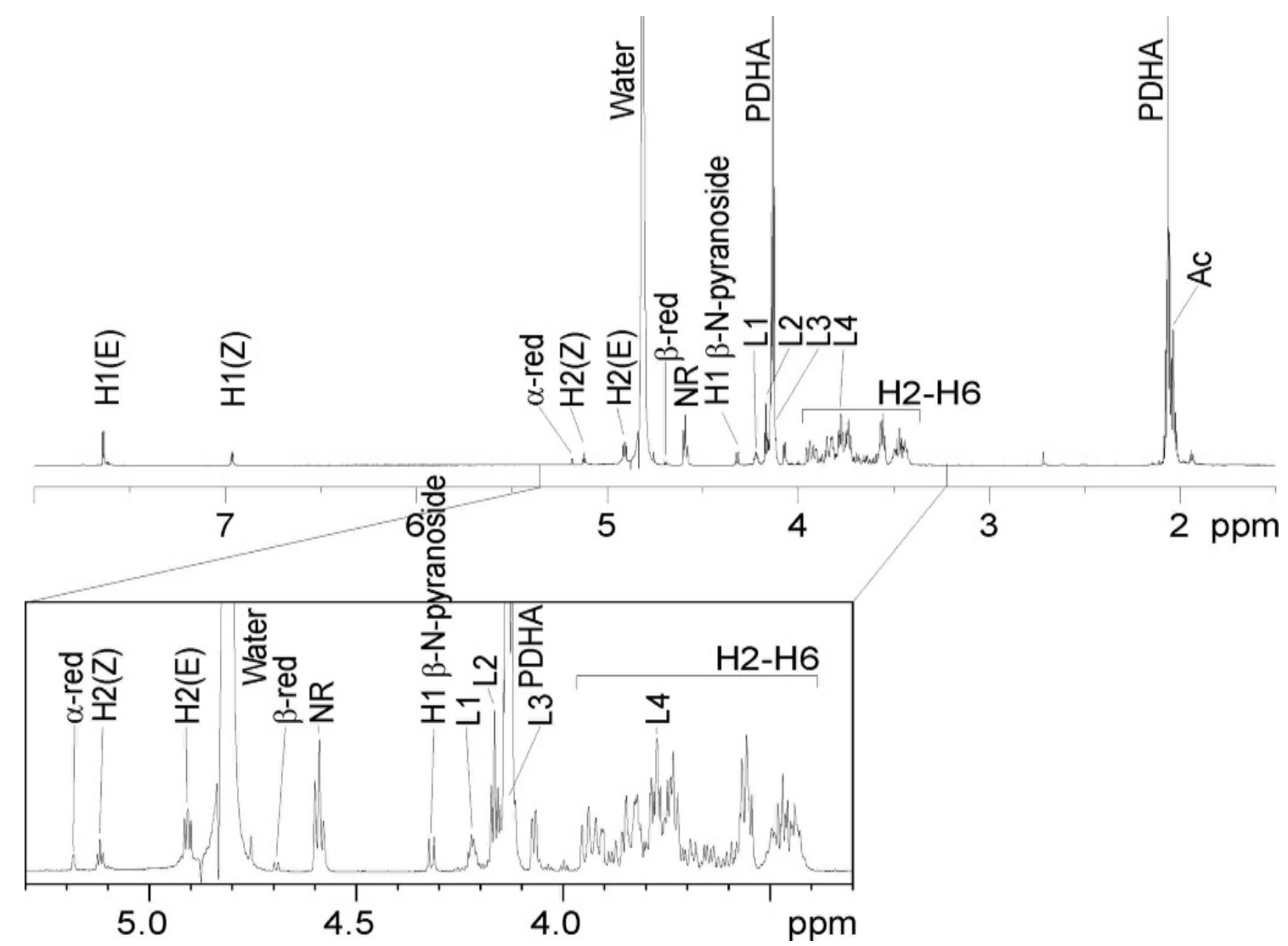

Figure S3: 1D proton spectrum of the mixture of products and reactants obtained for the conjugation of PDHA to AA after equilibrium was reached $(>48 \mathrm{~h}$ ) recorded at $298 \mathrm{~K}$.

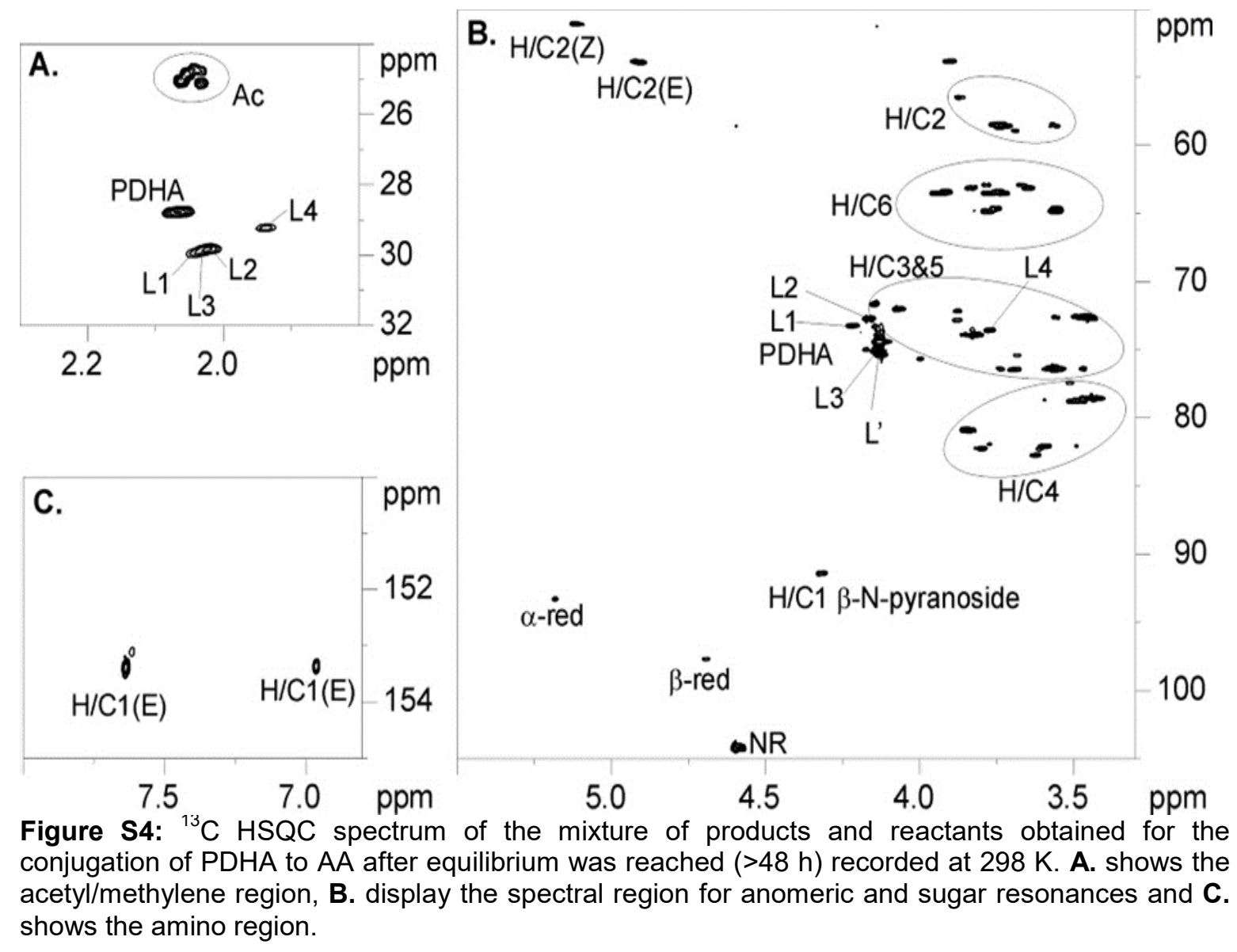


Table S1: Assignment of the chemical shifts for the mixture of products and reactants obtained for the conjugation of PDHA to AA after equilibrium was reached $(>48 \mathrm{~h}$ )

\begin{tabular}{|c|c|c|c|c|c|c|c|}
\hline \multirow[t]{2}{*}{ Structural unit } & \multicolumn{7}{|l|}{ Assignment } \\
\hline & H1; C1 & $\mathrm{H} 2 ; \mathrm{C} 2$ & H3; C3 & H4; C4 & H5; C5 & H6; C6 & $\mathrm{Ac}-\mathrm{H} ; \mathrm{C}$ \\
\hline (E)-oxime & $7.63 ; 153.4$ & $4.91 ; 53.7$ & $4.07 ; 72.0$ & $3.85 ; 80.8$ & $3.83 ; 73.9$ & $3.78,3.56 ; 64.9$ & $2.05 ; 24.7$ \\
\hline (Z)-oxime & $6.97 ; 153.2$ & $5.13 ; 50.9$ & $4.15 ; 71.4$ & $3.80 ; 82.3$ & $3.82 ; 73.9$ & $3.76,3.55 ; 64.6$ & $2.04 ; 25.1$ \\
\hline$\alpha$-red. & $5.18 ; 93.4$ & $3.88 ; 56.6$ & $3.88 ; 72.2$ & n.d & n.d & n.d & n.d \\
\hline$\beta$-red. & $4.69 ; 97.7$ & $3.69 ; 59.0$ & $3.52 ; 77.5$ & $3.62 ; 82.3$ & n.d & n.d & n.d \\
\hline NR & $4.60 ; 104.2$ & $3.74 ; 58.6$ & $3.56 ; 76.4$ & $3.44 ; 78.6$ & $3.46 ; 72.6$ & $3.93,3.74 ; 63.5$ & $2.06 ; 25.1$ \\
\hline \multirow[t]{2}{*}{$\beta-N$-pyranoside } & $4.32 ; 91.5$ & $3.90 ; 53.9$ & $3.69 ; 76.6$ & $3.59 ; 82.0$ & $3.50 ; 78.8$ & $3.83,3.65 ; 63.1$ & $2.05 ; 24.8$ \\
\hline & \multicolumn{2}{|c|}{$\begin{array}{l}\text { Methylene in PDHA } \\
\text { closest to conjugation }\end{array}$} & \multicolumn{2}{|c|}{$\begin{array}{l}\text { Middle methylene } \\
\text { in PDHA }\end{array}$} & \multicolumn{3}{|c|}{$\begin{array}{l}\text { Methylene in PDHA closest to } \\
\text { free oxyamine end }\end{array}$} \\
\hline L1 & \multicolumn{2}{|c|}{$4.22 ; 73.1$} & \multicolumn{2}{|c|}{$2.04 ; 29.9$} & \multicolumn{3}{|c|}{$4.14 ; 75.1$} \\
\hline L2 & \multicolumn{2}{|c|}{$4.16 ; 72.7$} & \multicolumn{2}{|c|}{$2.03 ; 29.8$} & \multicolumn{3}{|c|}{$4.14 ; 75.1$} \\
\hline L3 & \multicolumn{2}{|c|}{$4.12 ; 75.4$} & \multicolumn{2}{|c|}{$2.04 ; 30,0$} & \multicolumn{3}{|c|}{$4.14 ; 75.1$} \\
\hline L4 & \multicolumn{2}{|c|}{$3.77 ; 73.6$} & \multicolumn{2}{|c|}{$1.94 ; 29.3$} & \multicolumn{3}{|c|}{$4.14 ; 75.1$} \\
\hline
\end{tabular}

n.d: not determined

\section{S4 Pseudo first order kinetics modelling of the conjugation reactions}

All conjugation reactions were assumed to follow pseudo first order kinetics. By treating the conjugates as one reaction product, the experimental data were fitted to a first order kinetics model (Figure S5) using the following rate equations

$$
[O]+[X] \underset{k_{-1}}{\stackrel{k_{1}}{\rightleftarrows}}[X=O]
$$

where $[O]$ is the concentration of oligomers, $[X]$ is the concentration of PDHA or $A D H,[X=O]$ is the concentration of conjugates, $k_{1}$ is the rate constant for the conjugation and $k_{-1}$ is the rate constant for the reverse reaction. Hence, the rate of conjugation was determined from

$$
\frac{d[X=O]}{d t}=k_{1}[O][X]-k_{-1}[X=O]
$$

The concentration of conjugates at specific time points, $[X=O]_{t}$ was calculated from the rate by the following equation

$$
[X=O]_{t}=\frac{d[X=O]}{d t} \Delta t+[X=O]_{t-\Delta t}
$$


where, $\mathrm{t}$ is the time, and $\Delta \mathrm{t}$ is the time difference from last modelled time point. All the conjugation reactions were modelled using this approach.

a)

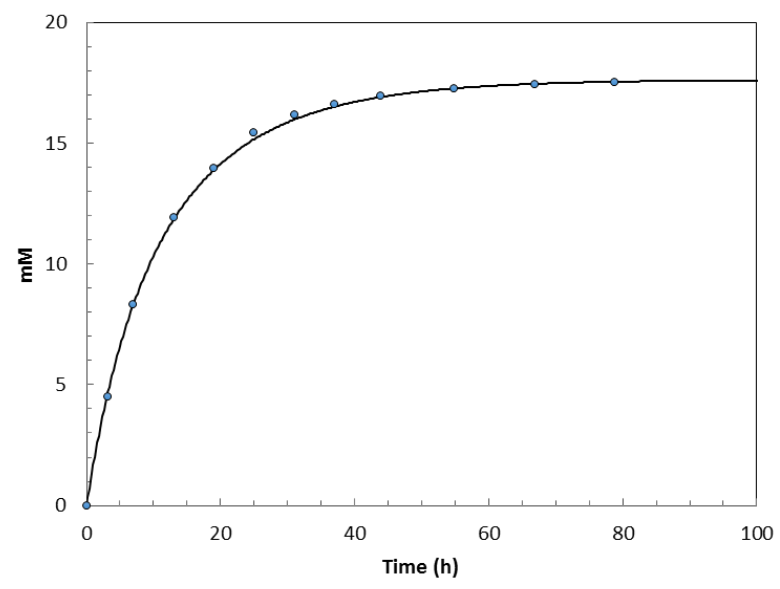

b)

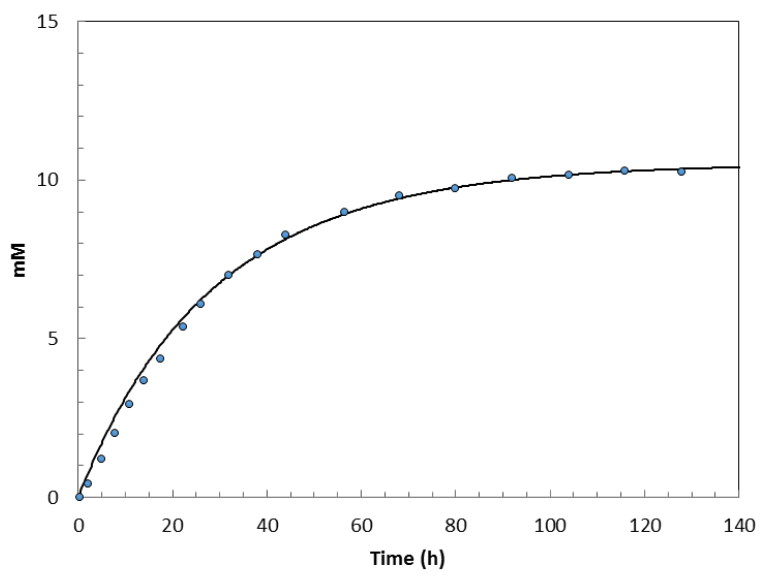

Figure S5: a) Model (solid line) fitted to the experimental data (blue dots, combined yield of conjugates in $\mathrm{mM}$ ) obtained for the conjugation of PDHA (2 equivalents) to $\mathrm{AA}$ at $\mathrm{pH} 4.0 \mathrm{~b}$ ) Model (solid line) fitted to the experimental data (blue dots combined yield of conjugates in $\mathrm{mM}$ ) obtained for the conjugation of $\mathrm{ADH}$ (2 equivalents) at $\mathrm{pH} 4.0$.

\section{S5 $\mathrm{pH}$ titration by ${ }^{1} \mathrm{H}-\mathrm{NMR}$ to determine the $\mathrm{pK}_{\mathrm{a}}$ of PDHA and ADH}

The $\mathrm{pK}_{\mathrm{a}}$ of the terminal amino groups of PDHA and $\mathrm{ADH}$ was determined by monitoring the change in chemical shifts for the methylene protons over a range of different $\mathrm{pH}$ values (Figure S6a and $\mathbf{c}$, respectively). The degree of dissociation ( $\alpha$ ) was calculated from the relative change in shifts going from fully protonated to deprotonated. The change in $\alpha$ as a function of $\mathrm{pH}$ was fitted to the Henderson-Hasselbalch equation (Eq. 6) providing a $\mathrm{pK}_{\mathrm{a}}$ of 4.2 and 3.1 for the free amine group of PDHA and ADH, respectively (Figure S6b and $\mathbf{d}$ ).

$$
p H=p K_{a}+\log \frac{\left[A^{-}\right]}{[H A]} \rightleftarrows p H=p K_{a}+\log \left(\frac{\alpha}{1-\alpha}\right)
$$



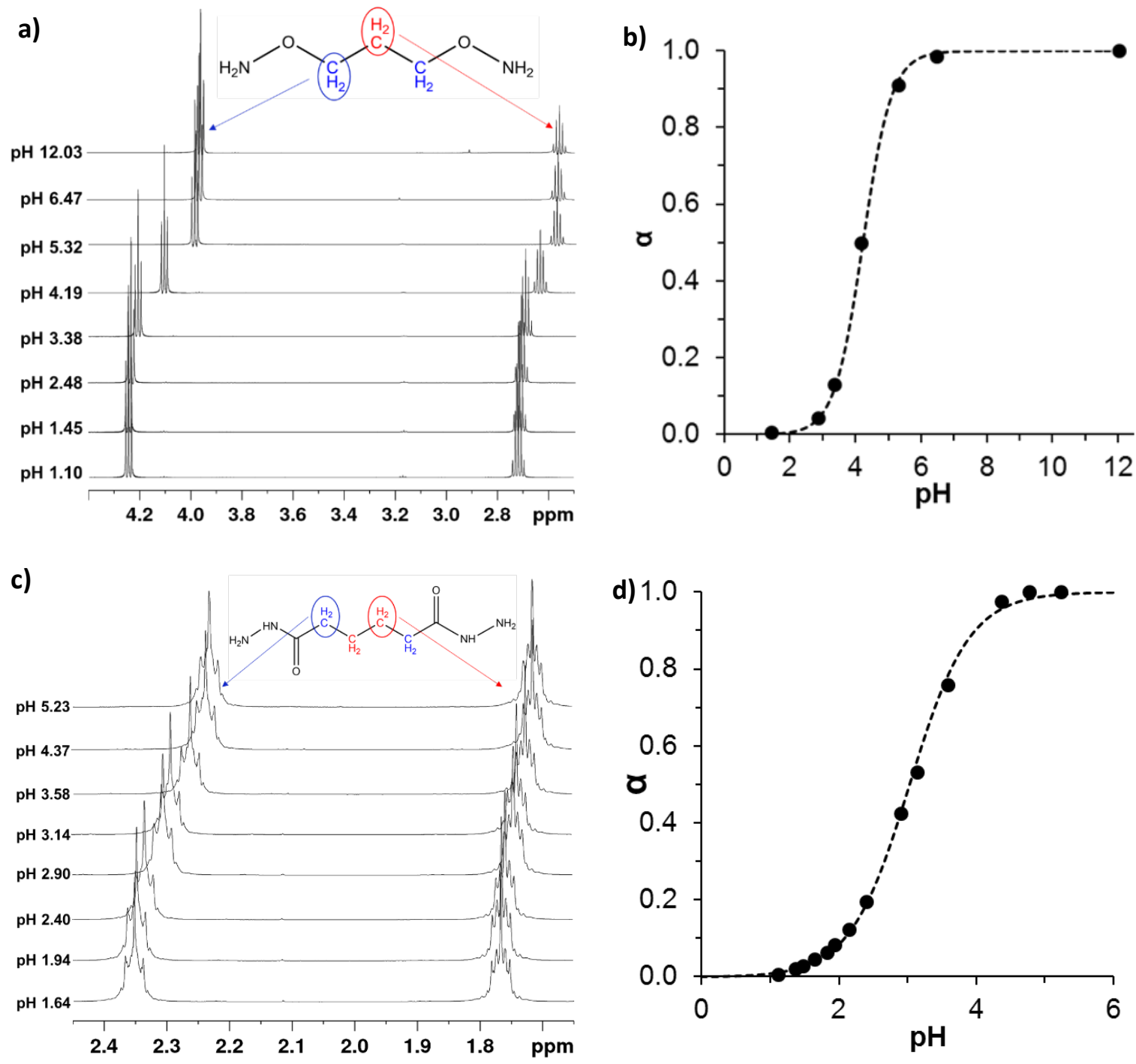

Figure S6: a) ${ }^{1} \mathrm{H}-\mathrm{NMR}$ spectra obtained for PDHA at different $\mathrm{pH}$ values $\mathbf{b}$ ) $\mathrm{pH}$ dependence of the chemical shits for the PDHA methylene protons, represented by the degree of dissociation calculated from the experimental data and fitted to the Henderson-Hasselbalch equation to obtain a $\mathrm{pK}_{\mathrm{a}}$ of 4.2 . c) ${ }^{1} \mathrm{H}-\mathrm{NMR}$ spectra obtained for $\mathrm{ADH}$ at different $\mathrm{pH}$ values d) $\mathrm{pH}$ dependence of the chemical shits for the ADH methylene protons, represented by the degree of dissociation calculated from the experimental data and fitted to the Henderson-Hasselbalch equation to obtain a $\mathrm{pK}_{\mathrm{a}}$ of 3.1.

\section{S6 ${ }^{1} \mathrm{H}-\mathrm{NMR}$ characterisation of the equilibrium mixtures for the conjugation reactions with AA}

The mixture of conjugates and unreacted oligomers at equilibrium for the reactions of $A A$ with 2 or 10 equivalents of PDHA or ADH at pH 3.0, 4.0 or 5.0 were characterized by ${ }^{1} \mathrm{H}-\mathrm{NMR}$. The spectra are given in Figure S7-S14. Yields (\%) were calculated by relating the integrals (not included) of the resonances resulting from $\mathrm{H} 1$ reducing end unit for the conjugates and unreacted oligomers at specific time points to the sum of these integrals $(100 \%$, theoretical yield). The following designations are used in the ${ }^{1} \mathrm{H}$-NMR spectra: $(E)$ - and $(Z)$-oxime $((E) /(Z)$-configuration of the oxime), $\beta$ - $N$-pyr. ( $\beta$ configuration of the $N$-pyranoside), $N$-glycoside (unidentified $N$-glycoside conjugate) and $\alpha$ - and $\beta$ red. ( $\alpha-/ \beta$-configuration of the anomeric proton in the reducing end $A$-unit). 


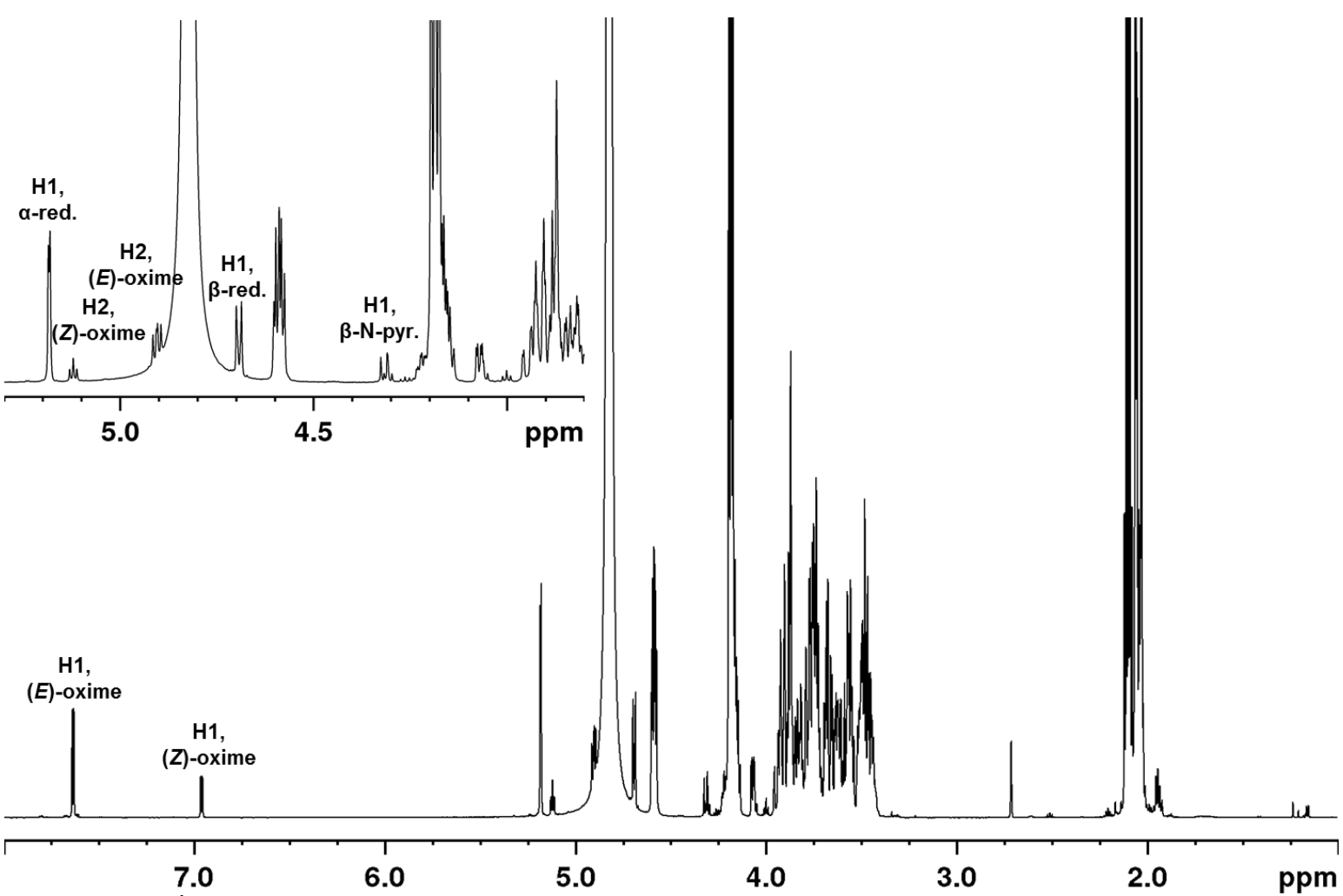

Figure S7: ${ }^{1} \mathrm{H}-\mathrm{NMR}$ characterisation of the mixture of conjugates and unreacted oligomers at equilibrium for the reaction of AA with 2 equivalents of PDHA in deuterated NaAc-buffer, $\mathrm{pH} 3.0$. The yield of conjugates at equilibrium was $33 \%$.

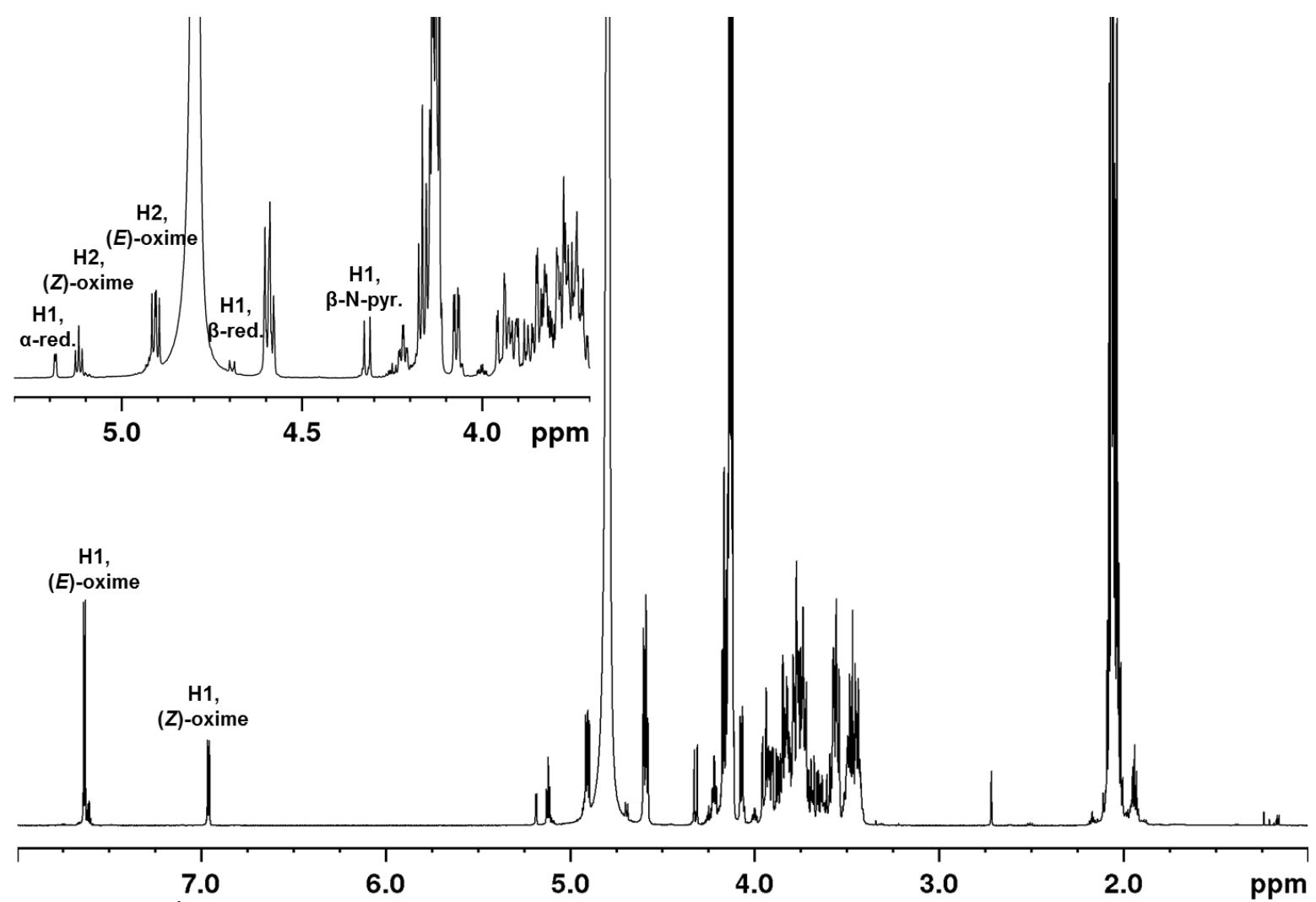

Figure S8: ${ }^{1} \mathrm{H}-\mathrm{NMR}$ characterisation of the mixture of conjugates and unreacted oligomers at equilibrium for the reaction of $\mathrm{AA}$ with 2 equivalents of PDHA in deuterated NaAc-buffer, $\mathrm{pH}$ 4.0. The yield of conjugates at equilibrium was $88 \%$. 

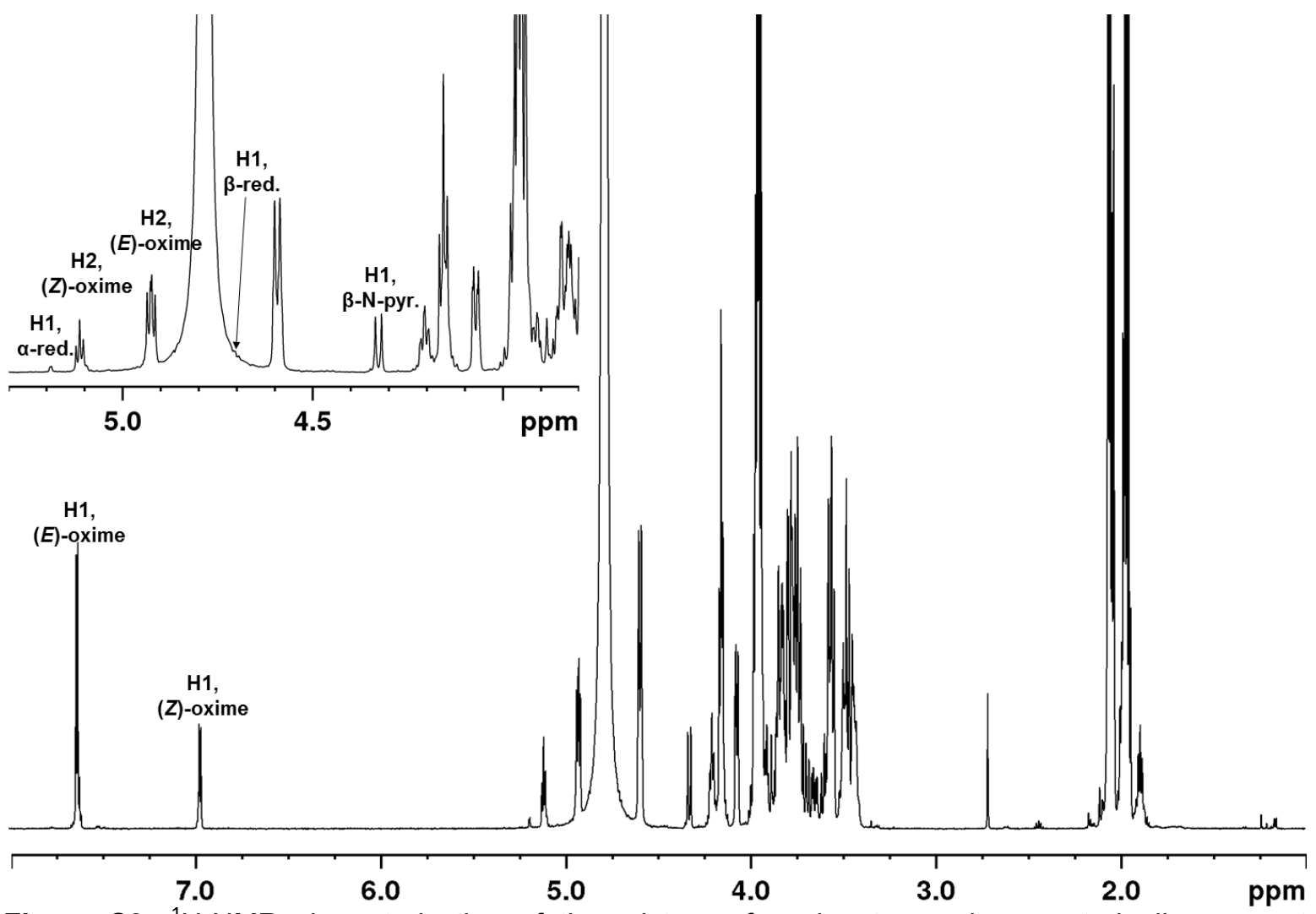

Figure S9: ${ }^{1} \mathrm{H}-\mathrm{NMR}$ characterisation of the mixture of conjugates and unreacted oligomers at equilibrium for the reaction of AA with 2 equivalents of PDHA in deuterated NaAc-buffer, pH 5.0. The yield of conjugates at equilibrium was $98 \%$.

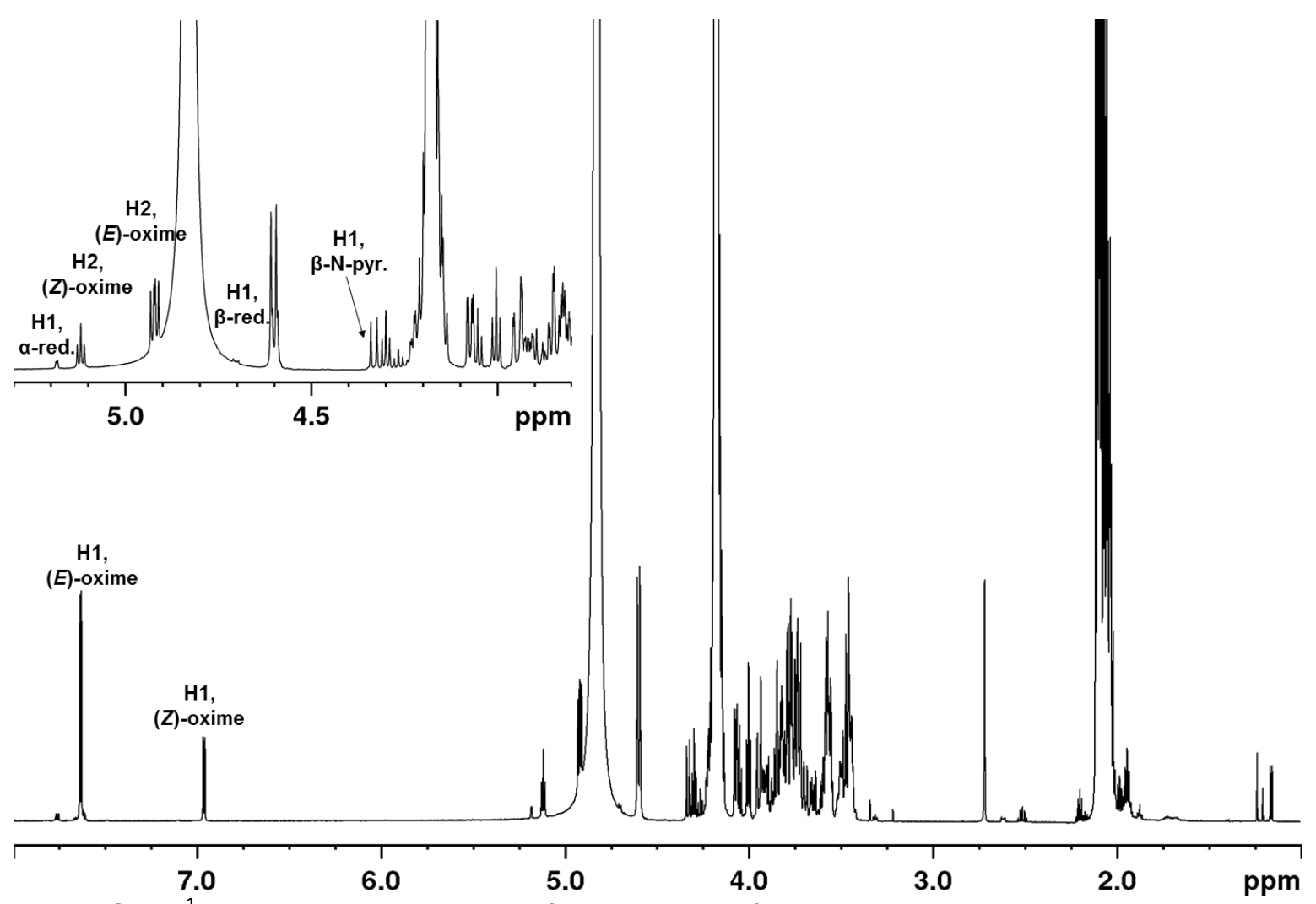

Figure S10: ${ }^{1} \mathrm{H}-\mathrm{NMR}$ characterisation of the mixture of conjugates and unreacted oligomers at equilibrium for the reaction of AA with 10 equivalents of PDHA in deuterated NaAc-buffer, pH 4.0. The yield of conjugates at equilibrium was $96 \%$. 


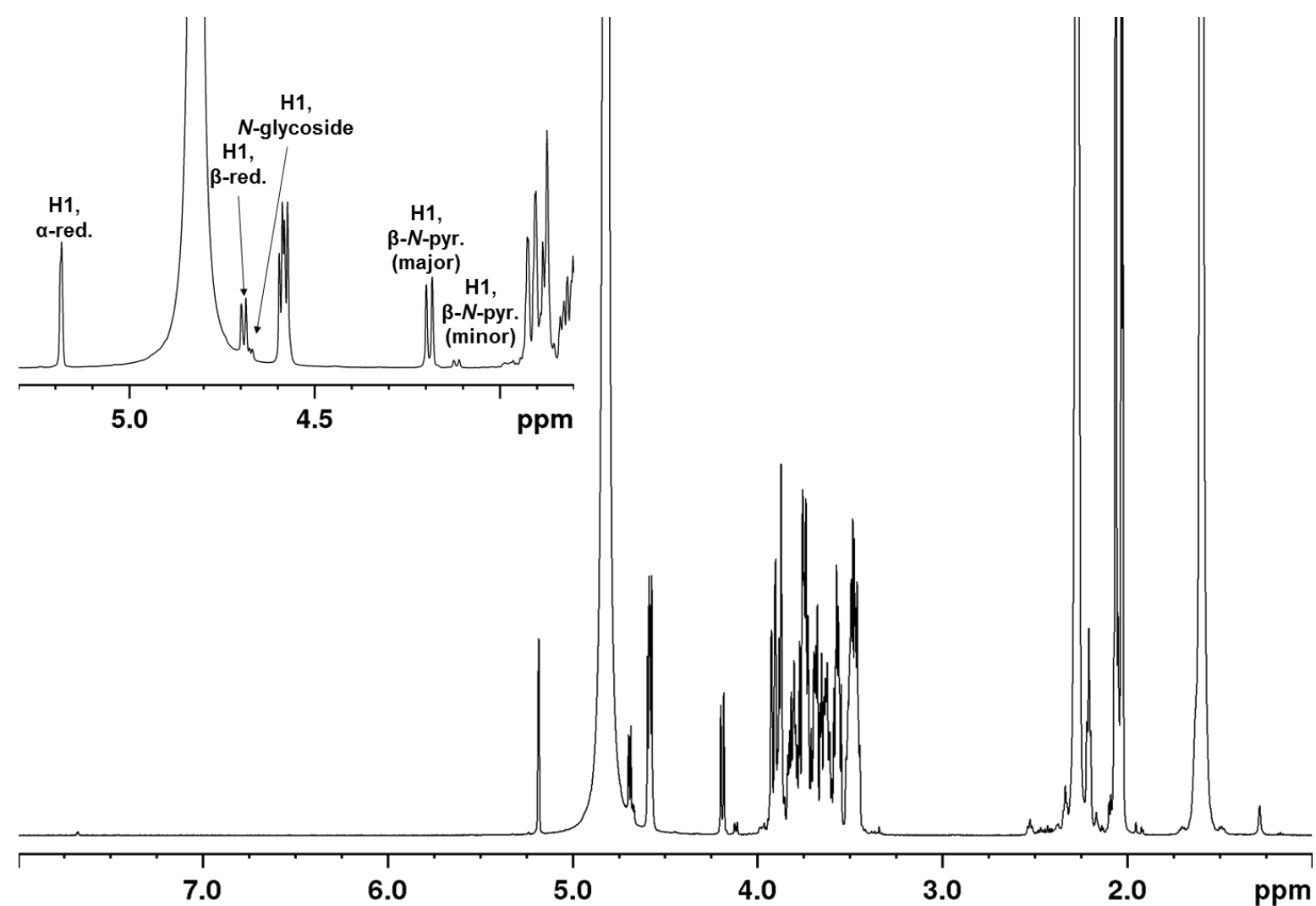

Figure S11: ${ }^{1} \mathrm{H}-\mathrm{NMR}$ characterisation of the mixture of conjugates and unreacted oligomers at equilibrium for the reaction of AA with 2 equivalents of $\mathrm{ADH}$ in deuterated $\mathrm{NaAc}-\mathrm{buffer}, \mathrm{pH}$ 3.0. The yield of conjugates at equilibrium was $46 \%$.
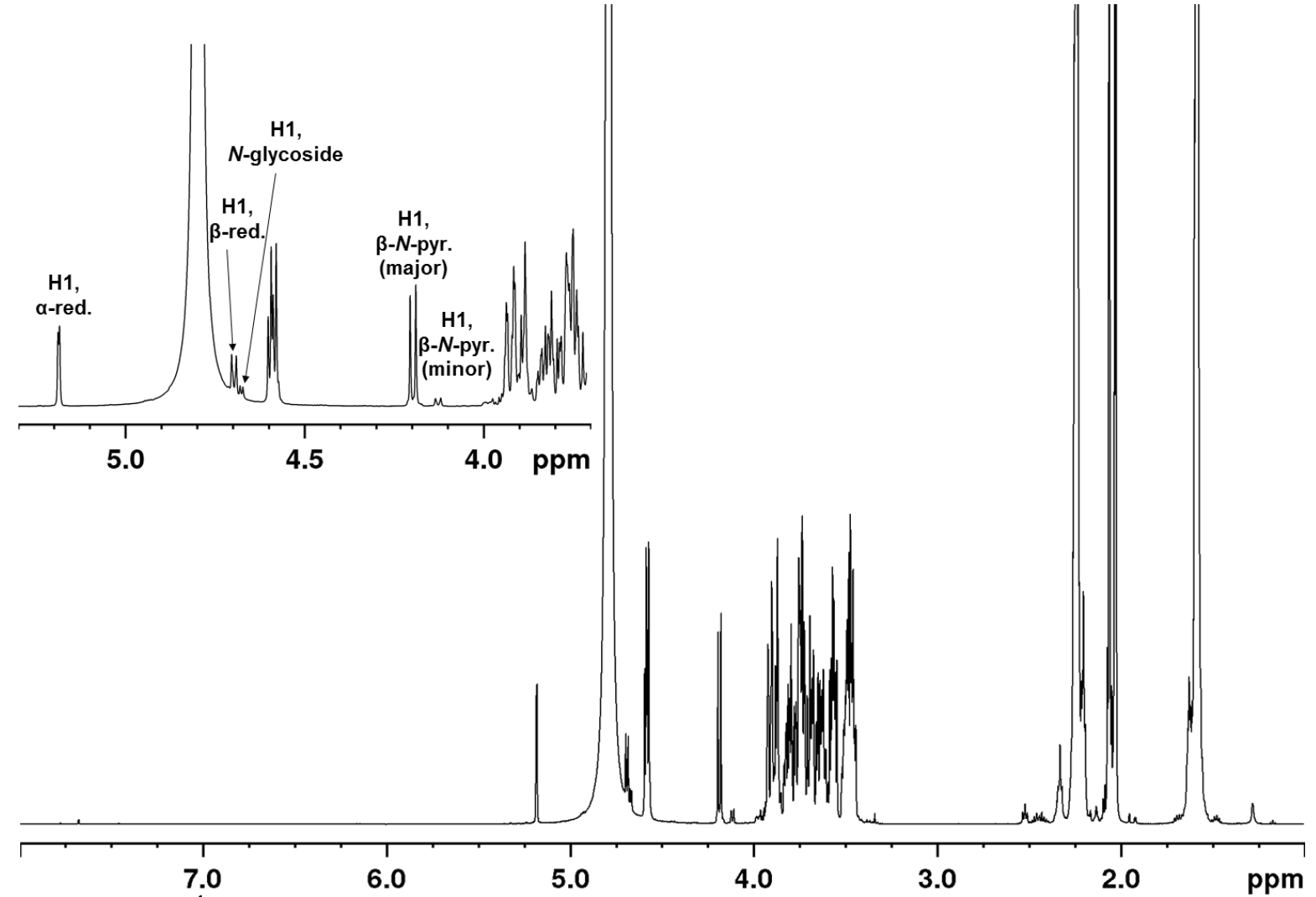

Figure S12: ${ }^{1} \mathrm{H}-\mathrm{NMR}$ characterisation of the mixture of conjugates and unreacted oligomers at equilibrium for the reaction of $A A$ with 2 equivalents of $A D H$ in deuterated NaAc-buffer, $\mathrm{pH}$ 4.0. The yield of conjugates at equilibrium was $53 \%$. 


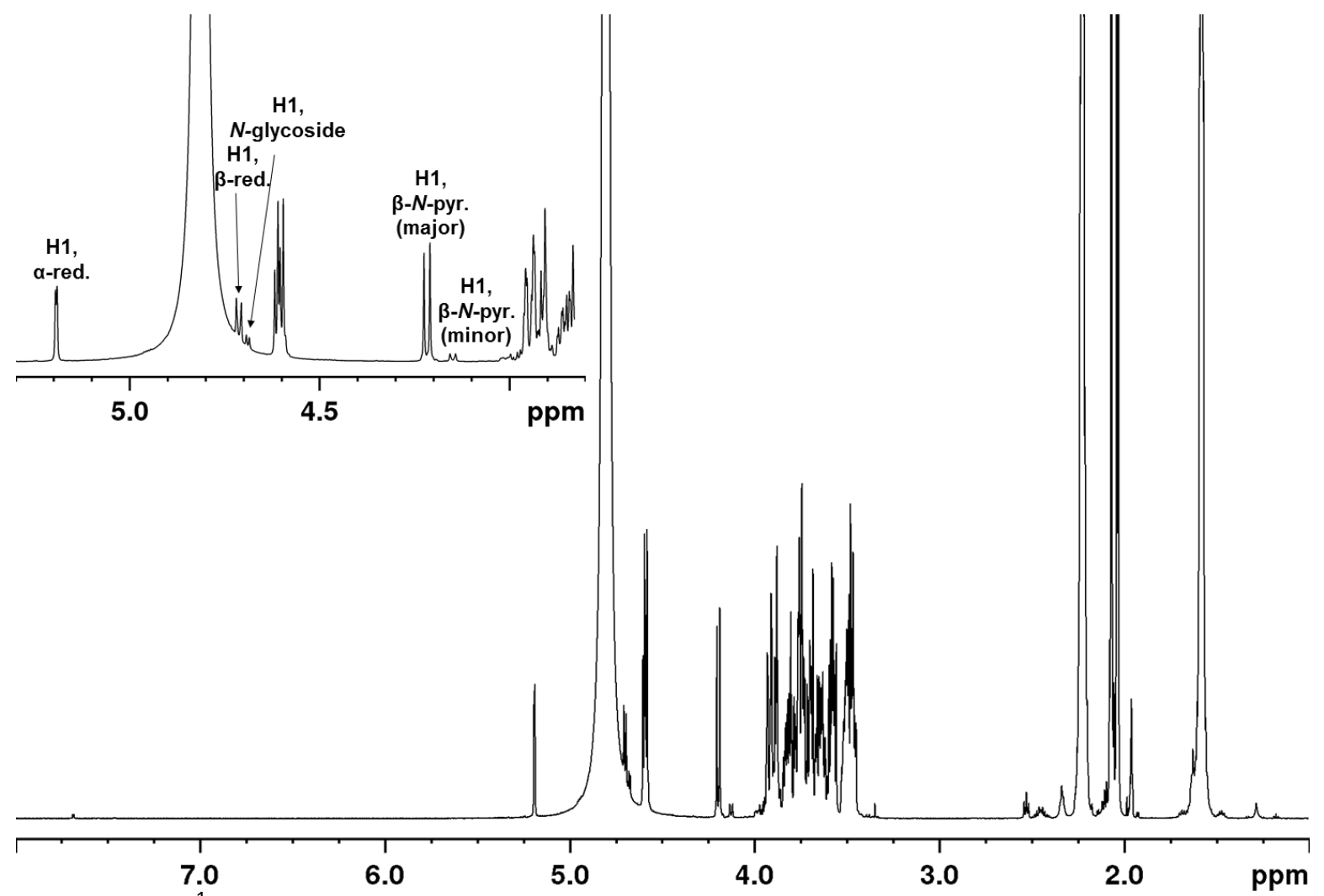

Figure S13: ${ }^{1} \mathrm{H}-\mathrm{NMR}$ characterisation of the mixture of conjugates and unreacted oligomers at equilibrium for the reaction of AA with 2 equivalents of $A D H$ in deuterated NaAc-buffer, pH 5.0. The yield of conjugates at equilibrium was $57 \%$.

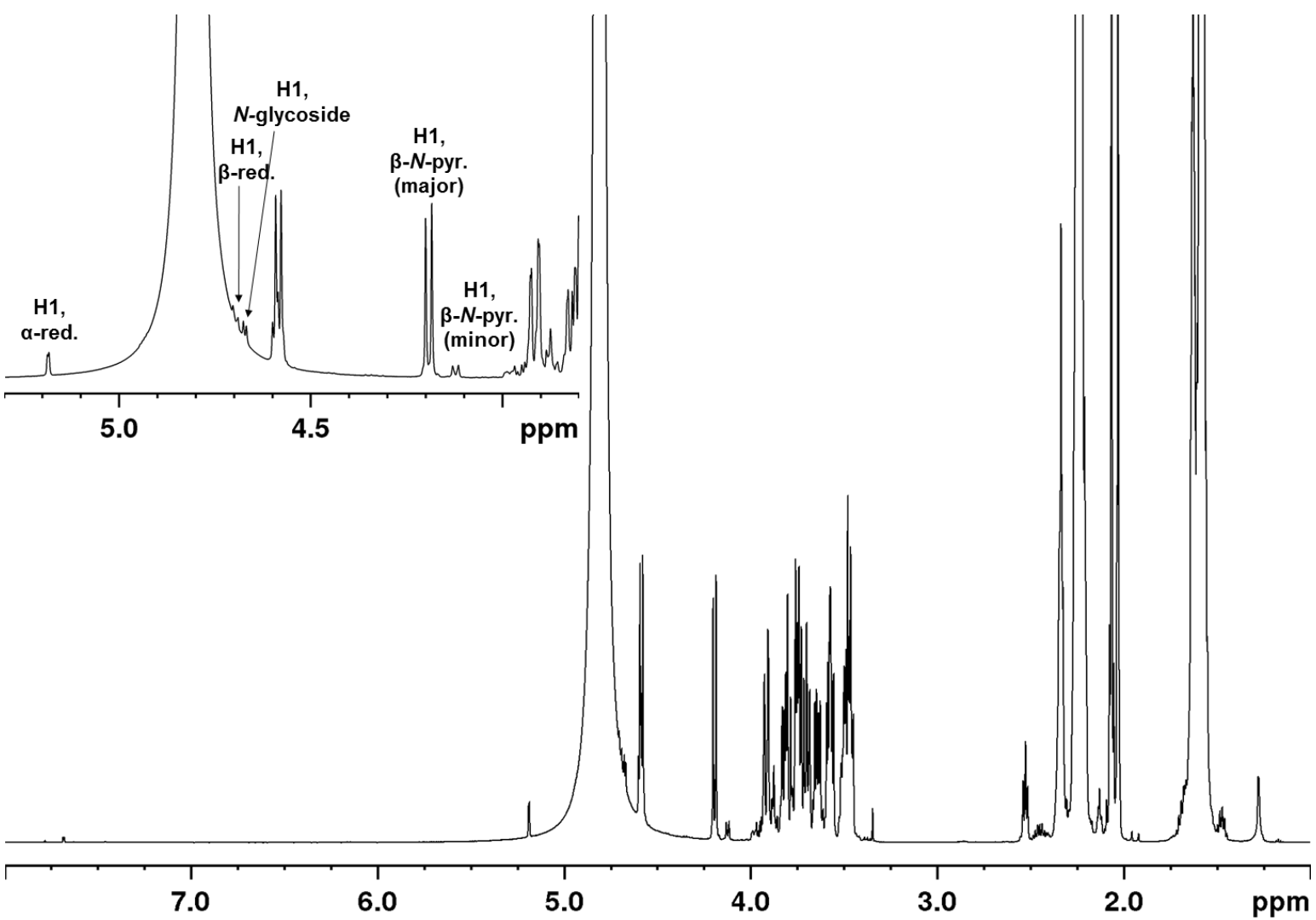

Figure S14: ${ }^{1} \mathrm{H}-\mathrm{NMR}$ characterisation of the mixture of conjugates and unreacted oligomers at equilibrium for the reaction of AA with 10 equivalents of $\mathrm{ADH}$ in deuterated $\mathrm{NaAc}$-buffer, $\mathrm{pH}$ 4.0. The yield of conjugates at equilibrium was $89 \%$. 


\section{S7 Comparison to $D$ at the reducing end}

The conjugation of PDHA and ADH (2 equivalents) to DD (chitobiose) was studied by time course

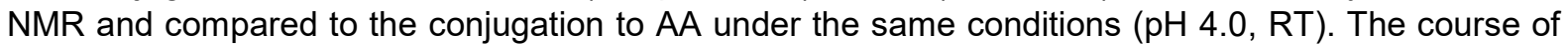
the reactions, presented by the combined yield of conjugates $(\%)$ at defined time points, are given in Figure S15.
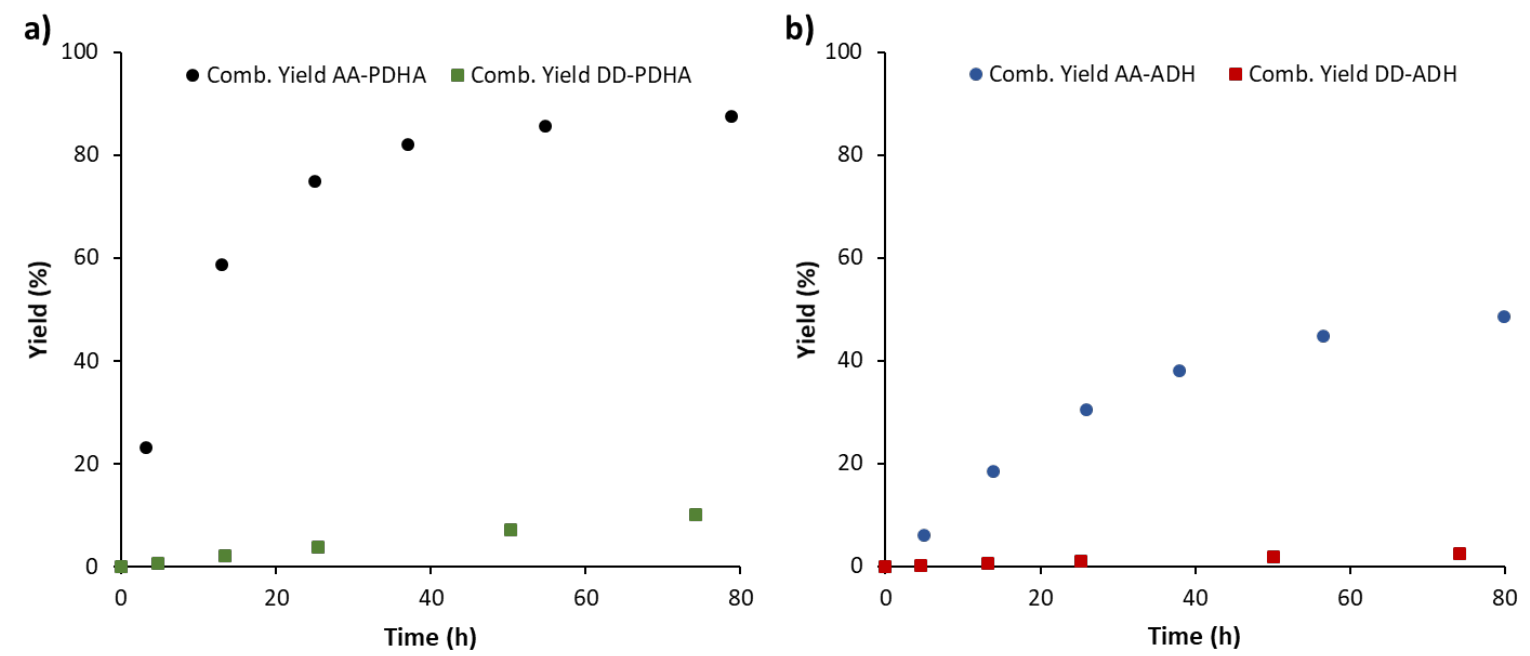

Figure S15: a) Course of the reaction for the conjugation of PDHA (2 equivalents) to $A A$ and DD at $\mathrm{pH} 4.0, \mathrm{RT}$ b) course of the reaction for the conjugation of $\mathrm{ADH}$ (2 equivalents) to $A A$ and $\mathrm{DD}$ at $\mathrm{pH}$ 4.0, RT.

\section{S8 Conjugation of PDHA and ADH to $D_{2} X A$ studied by time course NMR}

Conjugation of PDHA and $A D H$ to $D_{2} X A(X=$ either $A$ or $D)$ was studied by time course NMR. ${ }^{1} \mathrm{H}-\mathrm{NMR}$ spectra and the course of the reaction using 2 equivalents of PDHA and $A D H$, are given in Figure $\mathbf{S} 16$ and S17, respectively. Key resonances established for AA were detected in the spectra. The $D_{2} A A$ oligomers contained small amounts $(<15 \%)$ of $D_{2} D A$ (and opposite for the $D_{2} D A$ oligomers), appearing in the ${ }^{1} \mathrm{H}-\mathrm{NMR}$ spectra by side peaks for the reducing end resonances (e.g. $\mathrm{H} 1, \mathrm{\alpha}$-red. and $\mathrm{H} 1, \beta$-red). Yields (\%) were calculated by relating the integrals (not included) of the resonance resulting from the $\mathrm{H} 1$ reducing end unit for the conjugates and unreacted oligomers at specific time points to the sum of these integrals ( $100 \%$, theoretical yield). Yields, distribution of products and kinetics were in good agreement with the results obtained for the conjugation of PDHA and ADH to AA.

a)

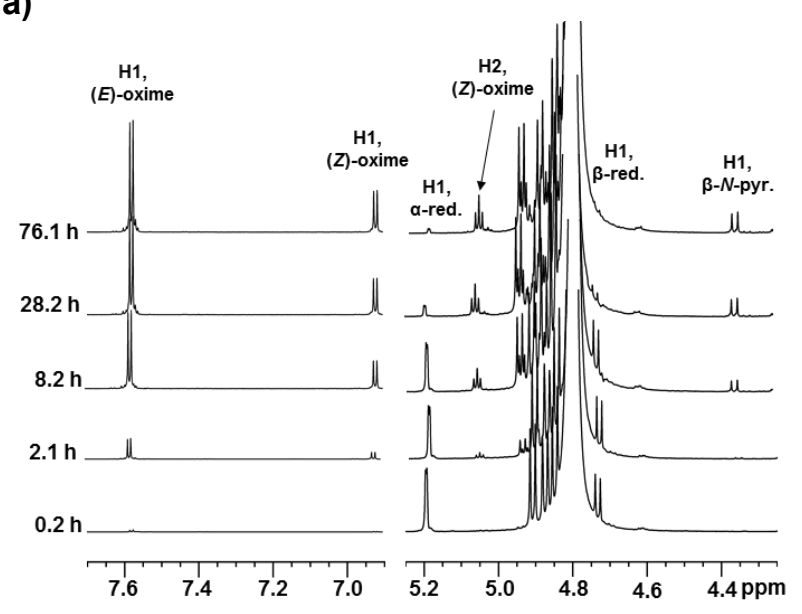

b)

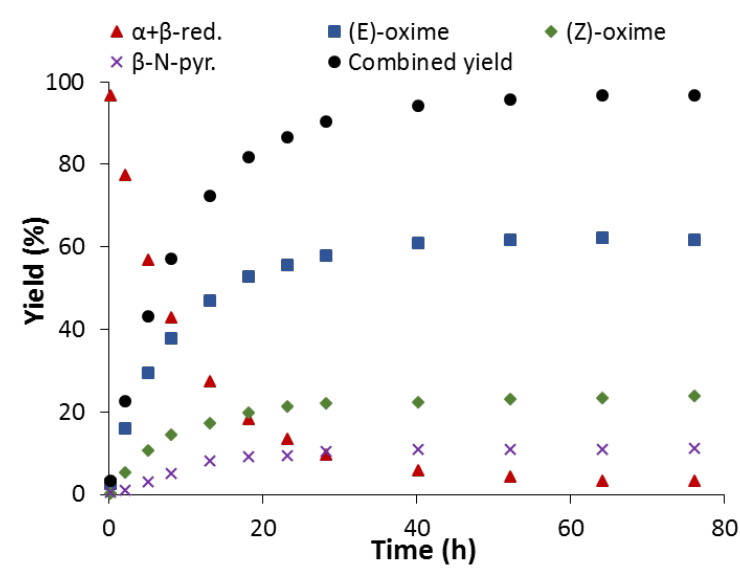

Figure S16: a) ${ }^{1} \mathrm{H}-\mathrm{NMR}$ spectra at defined time points for the conjugation of PDHA (2 equivalents) to $\mathrm{D}_{2} \mathrm{DA}, \mathrm{pH} 4.0 \mathrm{~b}$ ) course of the reaction obtained from the integration of the spectra shown in a). 
a)

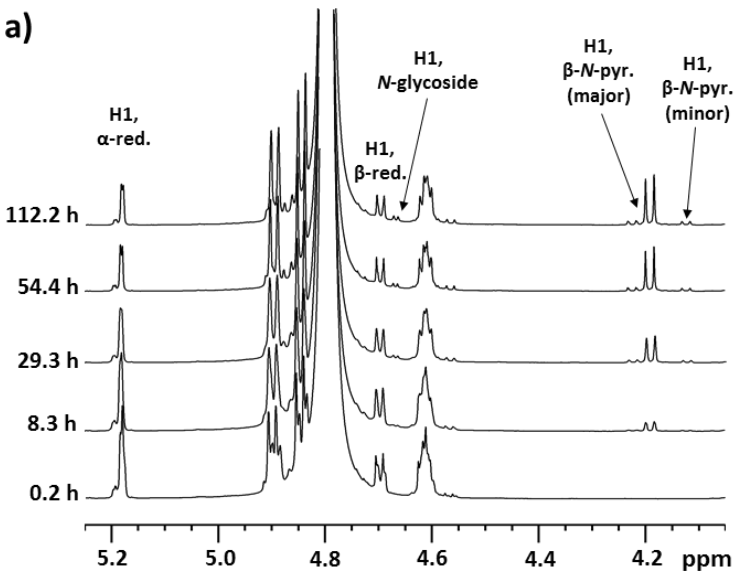

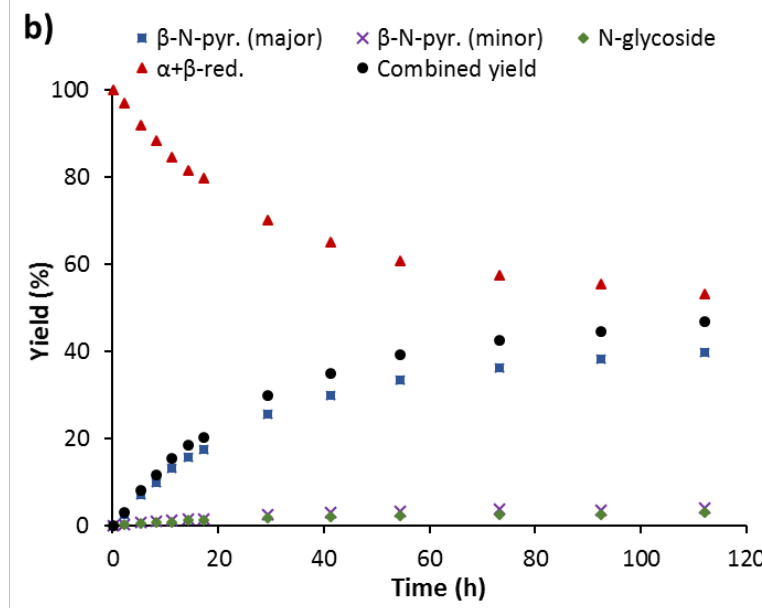

Figure S17: a) ${ }^{1} \mathrm{H}-\mathrm{NMR}$ spectra at defined time points for the reaction of $\mathrm{D}_{2} \mathrm{AA}$ with 2 equivalents of $\mathrm{ADH}$, at $\mathrm{pH} 4.0 \mathrm{~b}$ ) Course of the reaction obtained from the integration of the spectra shown in a).

\section{S9 ${ }^{1} \mathrm{H}-\mathrm{NMR}$ characterisation of the equilibrium mixtures for the conjugation reactions with $\mathrm{D}_{\mathrm{n}} \mathrm{XA}$ oligomers}

The mixture of conjugates and unreacted oligomers at equilibrium for the reactions of $D_{n} X A$ with 2 or 10 equivalents of PDHA or ADH at pH 4.0 were characterized by ${ }^{1} \mathrm{H}-\mathrm{NMR}$. The spectra are given in Figure S18-S21. Yields (\%) were calculated by relating the integrals (not included) of the resonances resulting from $\mathrm{H} 1$ reducing end unit of the conjugates and unreacted oligomers at specific time points to the sum of these integrals (100\%, theoretical yield). The following designations are used in the ${ }^{1} \mathrm{H}$ NMR spectra: $(E)$ - and (Z)-oxime $((E) /(Z)$-configuration of the oxime), $\beta-N$-pyr. ( $\beta$-configuration of the $\mathrm{N}$-pyranoside), $\mathrm{N}$-glycoside (unidentified $\mathrm{N}$-glycoside conjugate) and $\alpha$ - and $\beta$ - red. ( $\alpha$ - $/ \beta$-configuration of the anomeric proton in the reducing end A-unit). The double peaks observed for the $\mathrm{H} 1$ reducing end resonances (oligomers and conjugates) result from the different chemistries of oligomers having one or two $A$-units at the reducing end $\left(D_{n} D A\right.$ and $D_{n} A A$, respectively). 


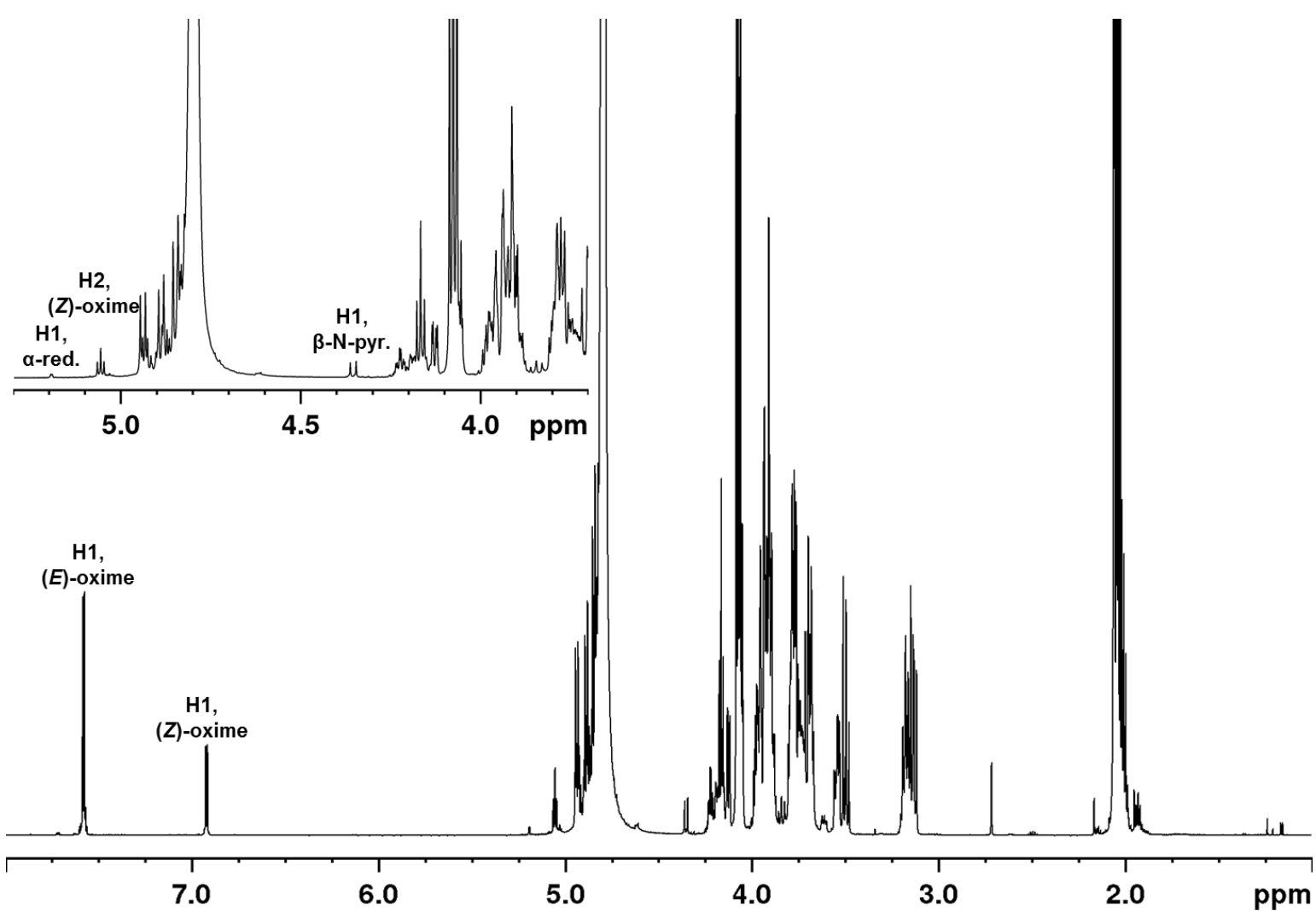

Figure S18: ${ }^{1} \mathrm{H}-\mathrm{NMR}$ characterisation of the mixture of conjugates and unreacted oligomers at equilibrium for the reaction of $\mathrm{D}_{2} \mathrm{DA}$ with 2 equivalents of PDHA in deuterated NaAc-buffer, $\mathrm{pH}$ 4.0. The yield of conjugates at equilibrium was $98 \%$.

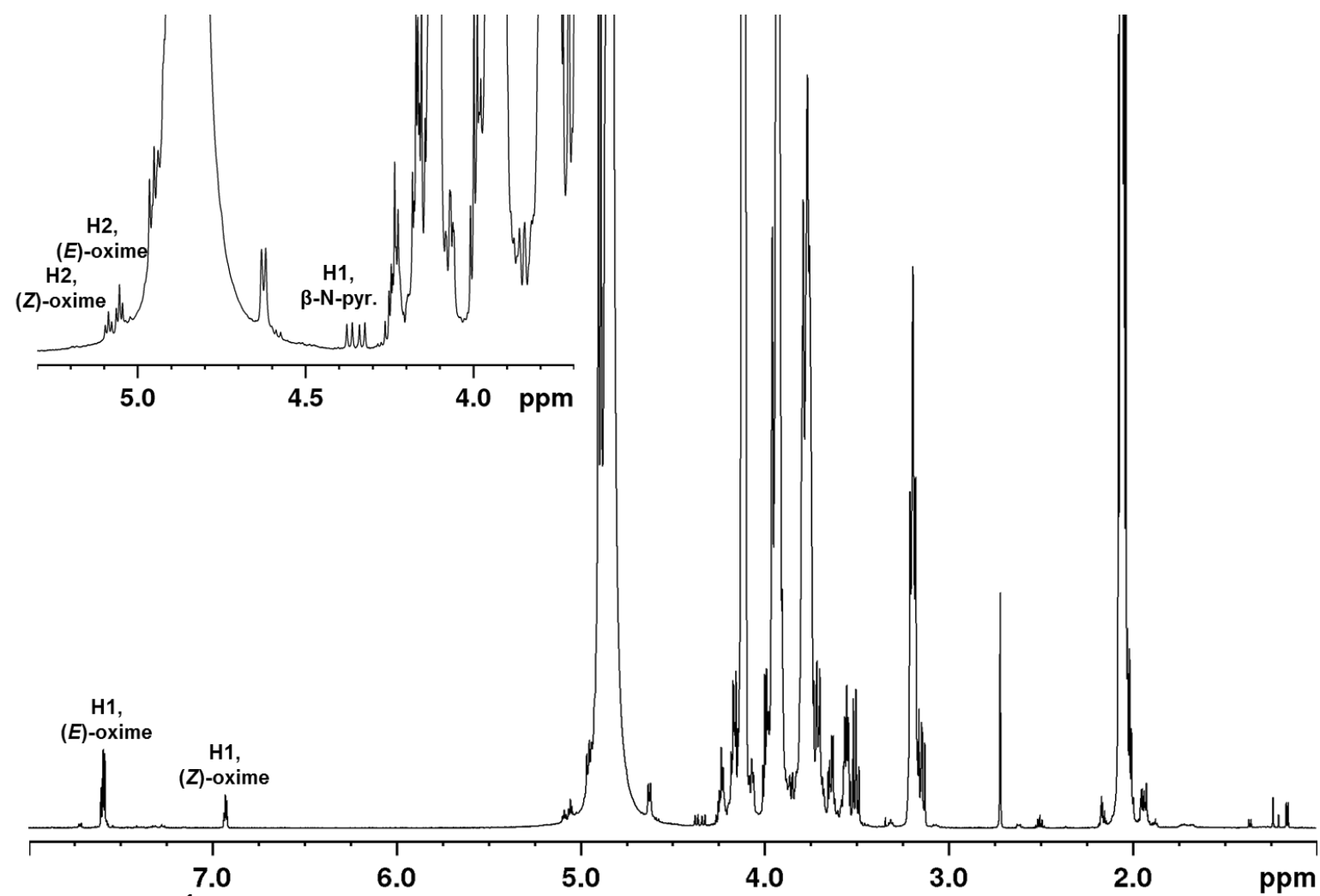

Figure S19: ${ }^{1} \mathrm{H}-\mathrm{NMR}$ characterisation of the mixture of conjugates and unreacted oligomers at equilibrium for the reaction of $\mathrm{D}_{8} \mathrm{XA}$ with 10 equivalents of PDHA in deuterated $\mathrm{NaAc}$-buffer, $\mathrm{pH} 4.0$. The yield of conjugates at equilibrium was $98 \%$. 


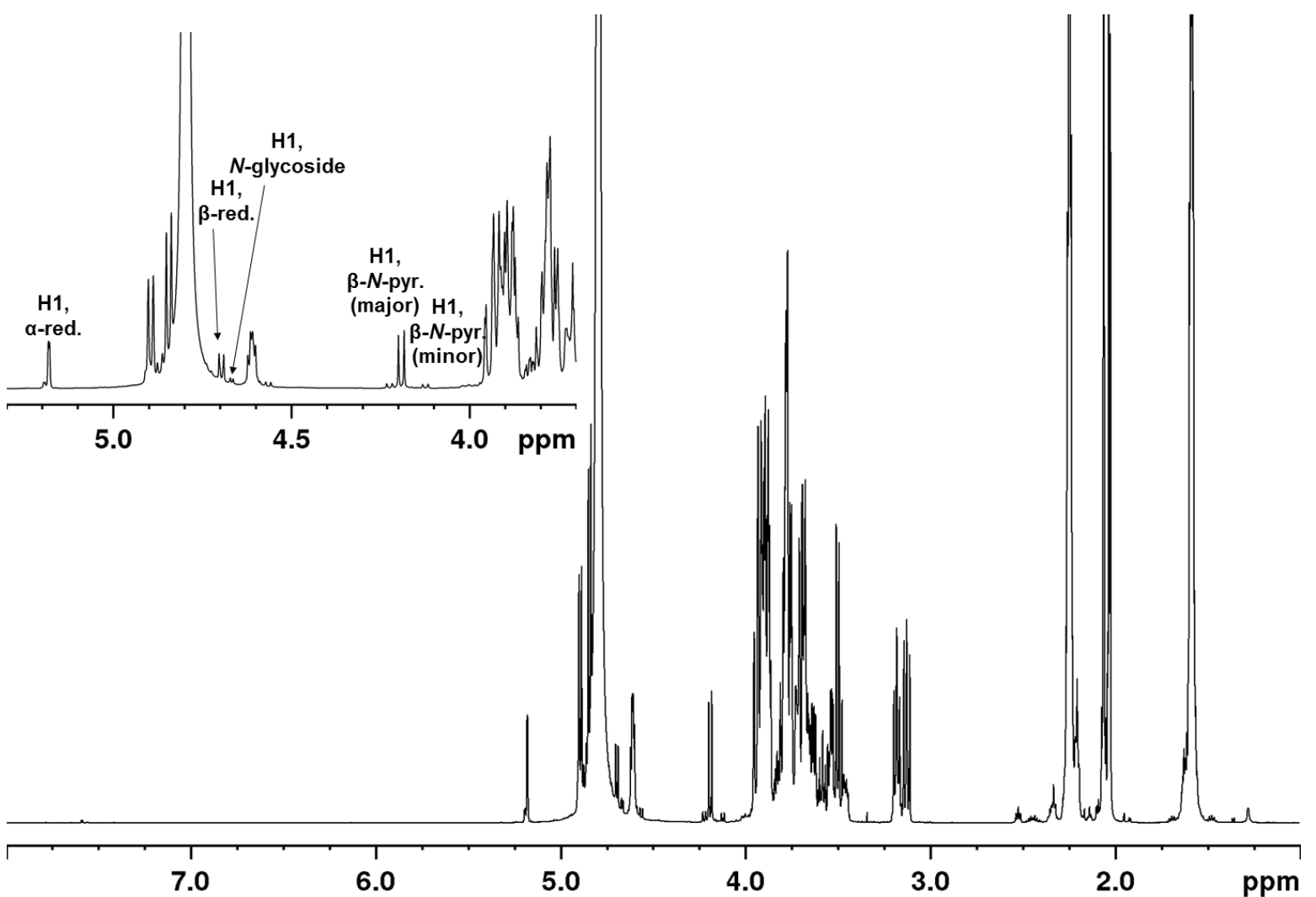

Figure S20: ${ }^{1} \mathrm{H}-\mathrm{NMR}$ characterisation of the mixture of conjugates and unreacted oligomers at equilibrium for the reaction of $D_{2} A A$ with 2 equivalents of $A D H$ in deuterated $\mathrm{NaAc}$-buffer, $\mathrm{pH} 4.0$. The yield of conjugates at equilibrium was $47 \%$.

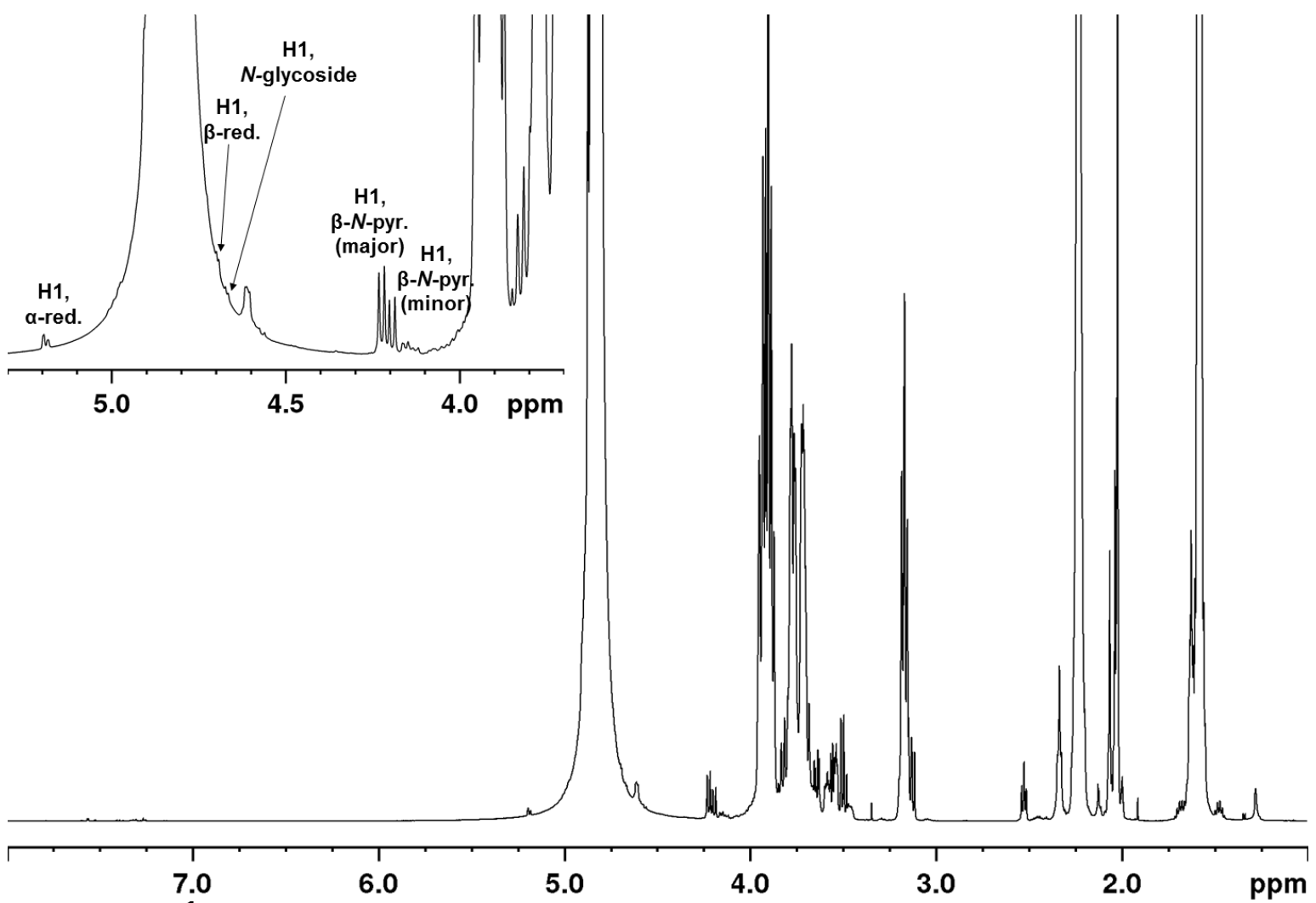

Figure S21: ${ }^{1} \mathrm{H}-\mathrm{NMR}$ characterisation of the mixture of conjugates and unreacted oligomers at equilibrium for the reaction of $D_{8} X A$ with 10 equivalents of $A D H$ in deuterated $\mathrm{NaAc}$-buffer, $\mathrm{pH}$ 4.0. The yield of conjugates at equilibrium was $86 \%$. 


\section{S10 ${ }^{1} H-N M R$ characterisation of the reaction mixtures for the $D_{n} X A-P D H A / A D H$ conjugates after 2 and 14 days of reduction}

Conjugates prepared with $\mathrm{D}_{2} \mathrm{AA}$ and 10 equivalents of PDHA or $\mathrm{ADH}$ at $\mathrm{pH} 4.0$ were subjected to reduction using 3 equivalents of $\mathrm{PB}$. The mixture of reduced secondary amine conjugates, nonreduced conjugates (oximes/hydrazones and $\mathrm{N}$-glycosides) and unreacted oligomers after 2 and 14 days of reduction where characterized by ${ }^{1} \mathrm{H}-\mathrm{NMR}$. The spectra are given in Figure S22 and S23. The following designations are used for the assignment in the obtained ${ }^{1} \mathrm{H}-\mathrm{NMR}$ spectra: $(E)$ - and $(Z)$ oxime $((E) /(Z)$-configurations of the oxime), $\beta-N$-pyr. ( $\beta$-configuration of the $N$-pyranoside), $N$ glycoside (unidentified $N$-glycoside conjugate), $\alpha$ - and $\beta$ - red. ( $\alpha$-/ $\beta$-configuration of the anomeric proton in the reducing end A-unit of the unreacted oligomer) and secondary amine (reduced secondary amine conjugates). Yields (\%) were calculated by relating the integrals (not included) of the resonances resulting from the $\mathrm{H} 1$ reducing end unit for the secondary amines, conjugates and unreacted oligomers at specific time points to the sum of these integrals at the start of the reduction (100\%, theoretical yield). The yield of unidentified products was calculated as the difference between the obtained and theoretical yield. In addition, resonances resulting from PB both in its reduced ( $r$ ) and oxidized form (in the area 7.3 to $8.7 \mathrm{ppm}$ ) are assigned in the spectra.

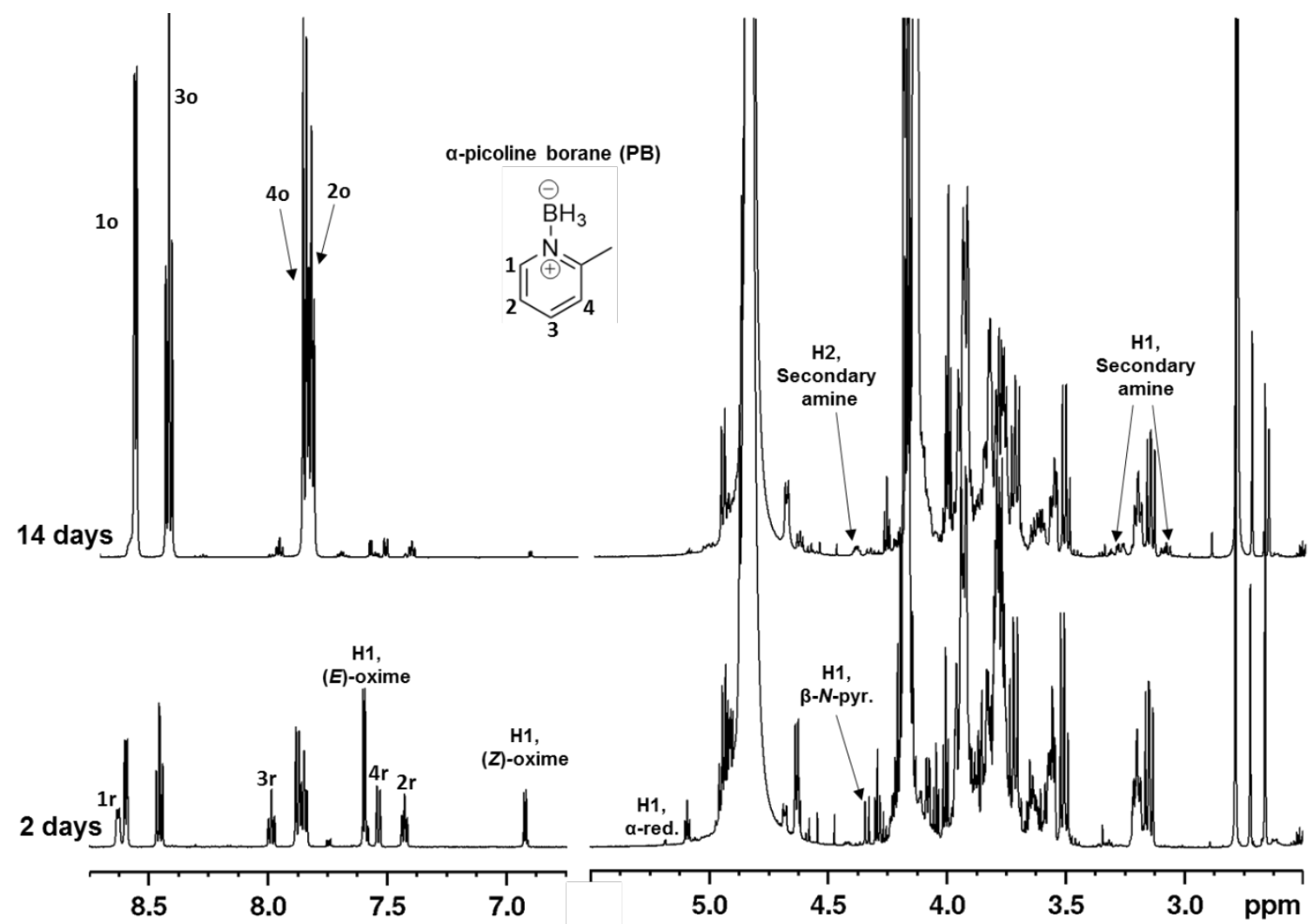

Figure S22: ${ }^{1} \mathrm{H}-\mathrm{NMR}$ characterisation of the mixture of secondary amines, conjugates and unreacted oligomers obtained after 2 and 14 days for the reduction of DDAA-PDHA conjugates (prepared using 2 equivalents $\mathrm{PDHA}$ ) at $\mathrm{pH} 4.0$ using 3 equivalents $\mathrm{PB}$. 


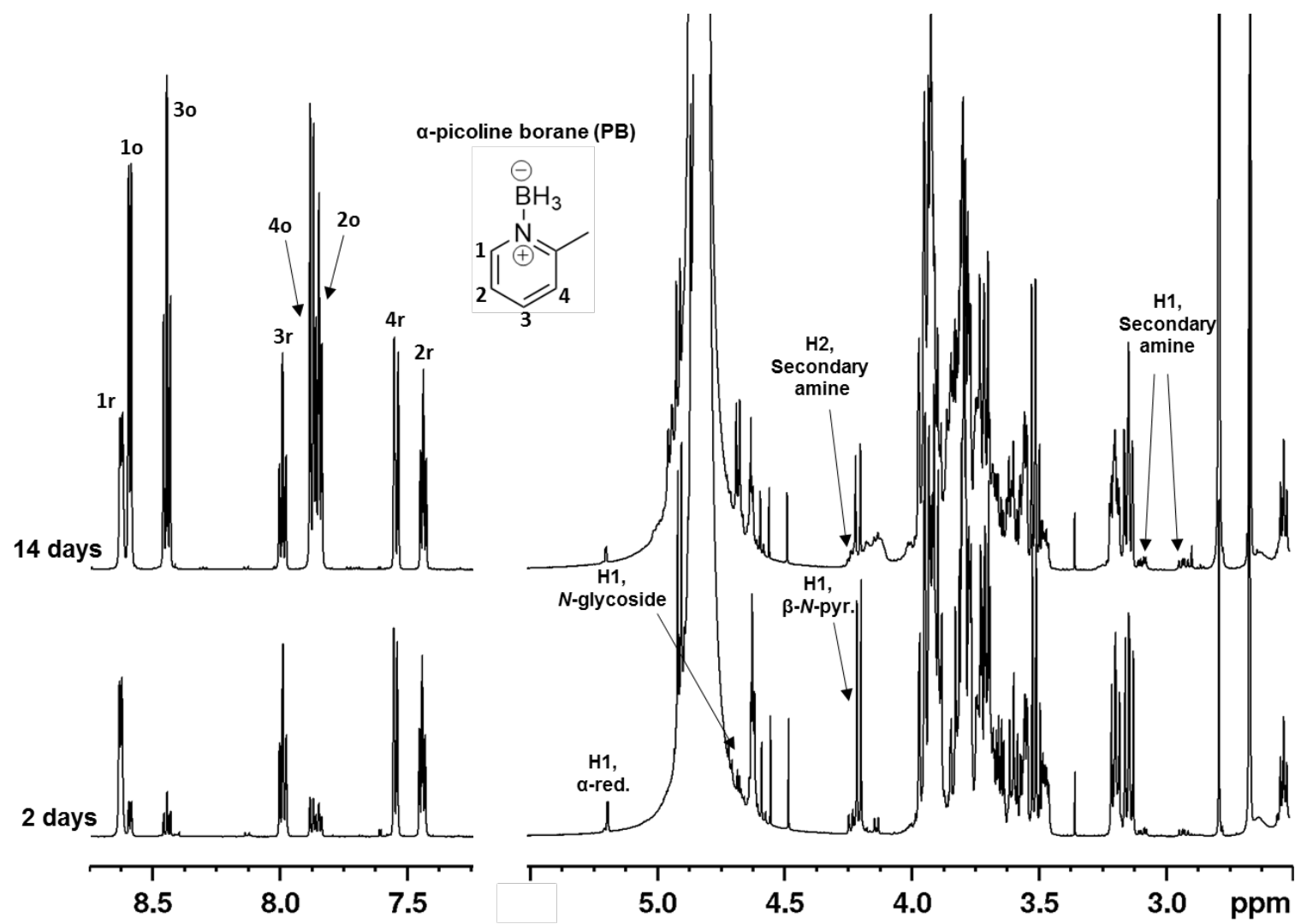

Figure S23: ${ }^{1} \mathrm{H}-\mathrm{NMR}$ characterisation of the mixture of secondary amines, conjugates and unreacted oligomers obtained after 2 and 14 days for the reduction of DDAA-ADH conjugates (prepared using 2 equivalents $\mathrm{ADH}$ ) at $\mathrm{pH} 4.0$ using 3 equivalents $\mathrm{PB}$.

S11 ${ }^{1} \mathrm{H}-\mathrm{NMR}$ and MS characterisation of products formed in the reduction of AA-PDHA and AAADH conjugates using $P B$

The products formed in the reduction of AA-PDHA and AA-ADH conjugates using PB (semipreparative scale) were fractionated by gel filtration chromatography (Figure S24). All fractions (products) were characterized by MS (Table S2) and the main products where characterized by ${ }^{1} \mathrm{H}$ NMR (Figure S25-S28). 


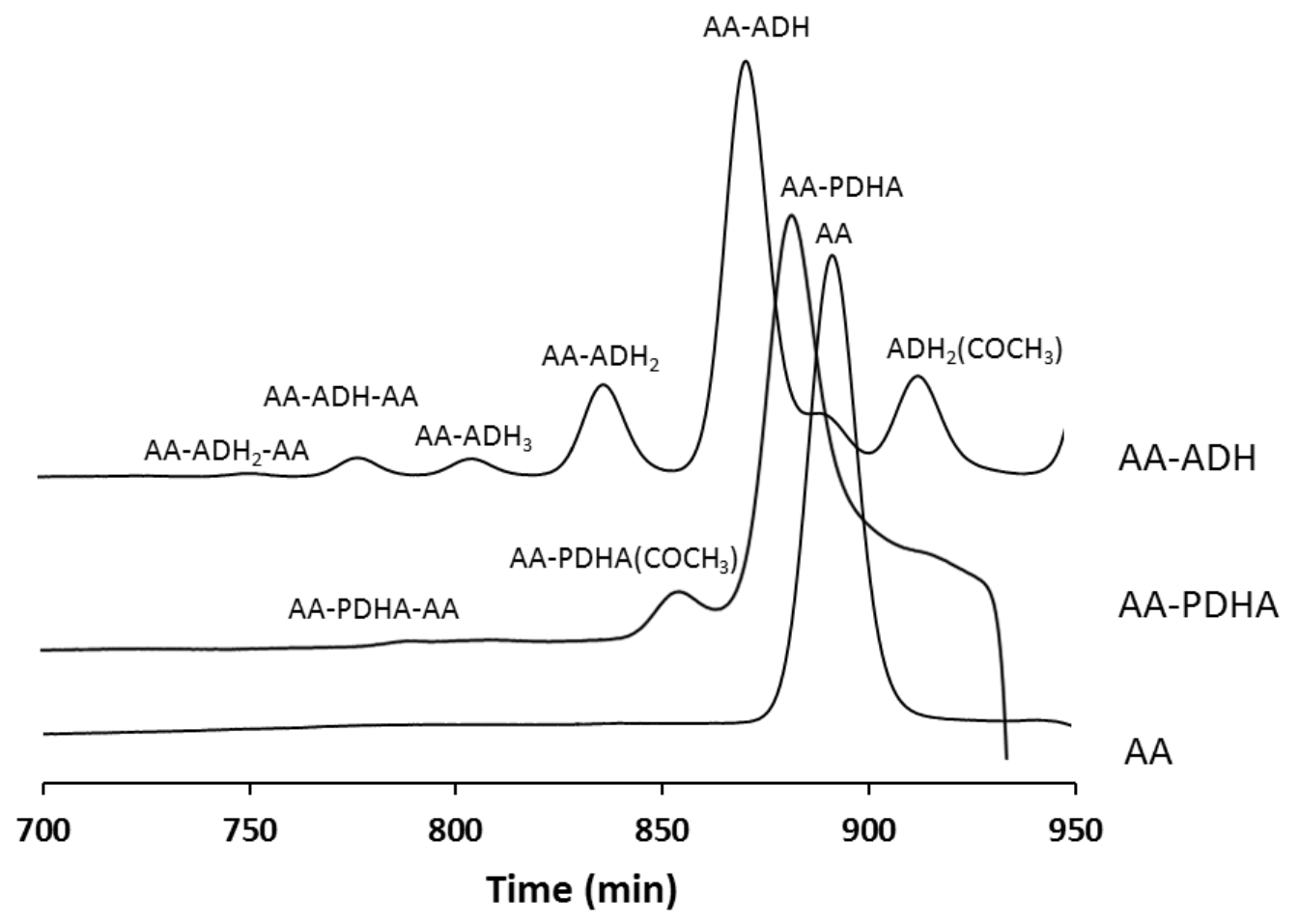

Figure S24: Fractionation of the mixture of products formed following conjugation and reduction of AA-PDHA and AA-ADH by gel filtration at $\mathrm{pH}$ 6.9. Fractionation of AA is included as a reference.

Table S2: Characterisation of the fractions from Figure S24 by MS.

\begin{tabular}{|c|c|c|c|c|c|c|c|}
\hline \multirow[b]{2}{*}{ Reaction } & \multirow[b]{2}{*}{ Fraction } & \multicolumn{6}{|c|}{ lons $(m / z)$} \\
\hline & & {$\left[\mathrm{M}^{+}\right.$} & {$[\mathrm{M}] \mathrm{H}^{-}$} & {$[\mathrm{M}] \mathrm{H}^{+}$} & {$[\mathrm{M}] \mathrm{Na}^{+}$} & {$[\mathrm{M}] \mathrm{Cl}^{-}$} & {$[\mathrm{M}] \mathrm{K}^{+}$} \\
\hline \multirow{3}{*}{ AA-PDHA } & AA-PDHA & 514.25 & 513.24 & 515.26 & 537.24 & 549.22 & 553.21 \\
\hline & AA-PDHA $\left(\mathrm{COCH}_{3}\right)$ & 556.26 & 555.25 & 557.27 & 579.25 & 591.23 & 595.22 \\
\hline & AA-PDHA-AA & 922.42 & 921.41 & 923.43 & 945.41 & 957.39 & 961.38 \\
\hline \multirow{7}{*}{ AA-ADH } & $\mathrm{ADH}_{2}\left(\mathrm{COCH}_{3}\right)$ & 358.20 & 357.19 & 359.20 & 381.19 & - & 397.16 \\
\hline & $A A$ & 426.18 & 425.18 & 427.19 & 449.17 & 461.15 & 465.14 \\
\hline & AA-ADH & 582.28 & 581.28 & 583.29 & 605.27 & - & 621.24 \\
\hline & $\mathrm{AA}-\mathrm{ADH}_{2}$ & 724.36 & 723.35 & 725.37 & 747.35 & 759.33 & 763.32 \\
\hline & $\mathrm{AA}-\mathrm{ADH}_{3}$ & 866.43 & 865.43 & 867.44 & 889.42 & - & 905.39 \\
\hline & AA-ADH-AA & 988.44 & 987.43 & 989.45 & 1011.44 & 1023.41 & - \\
\hline & $\mathrm{AA}-\mathrm{ADH}_{2}-\mathrm{AA}$ & 1130.52 & 1129.51 & 1131.53 & 1153.51 & 1165.49 & 1169.48 \\
\hline
\end{tabular}




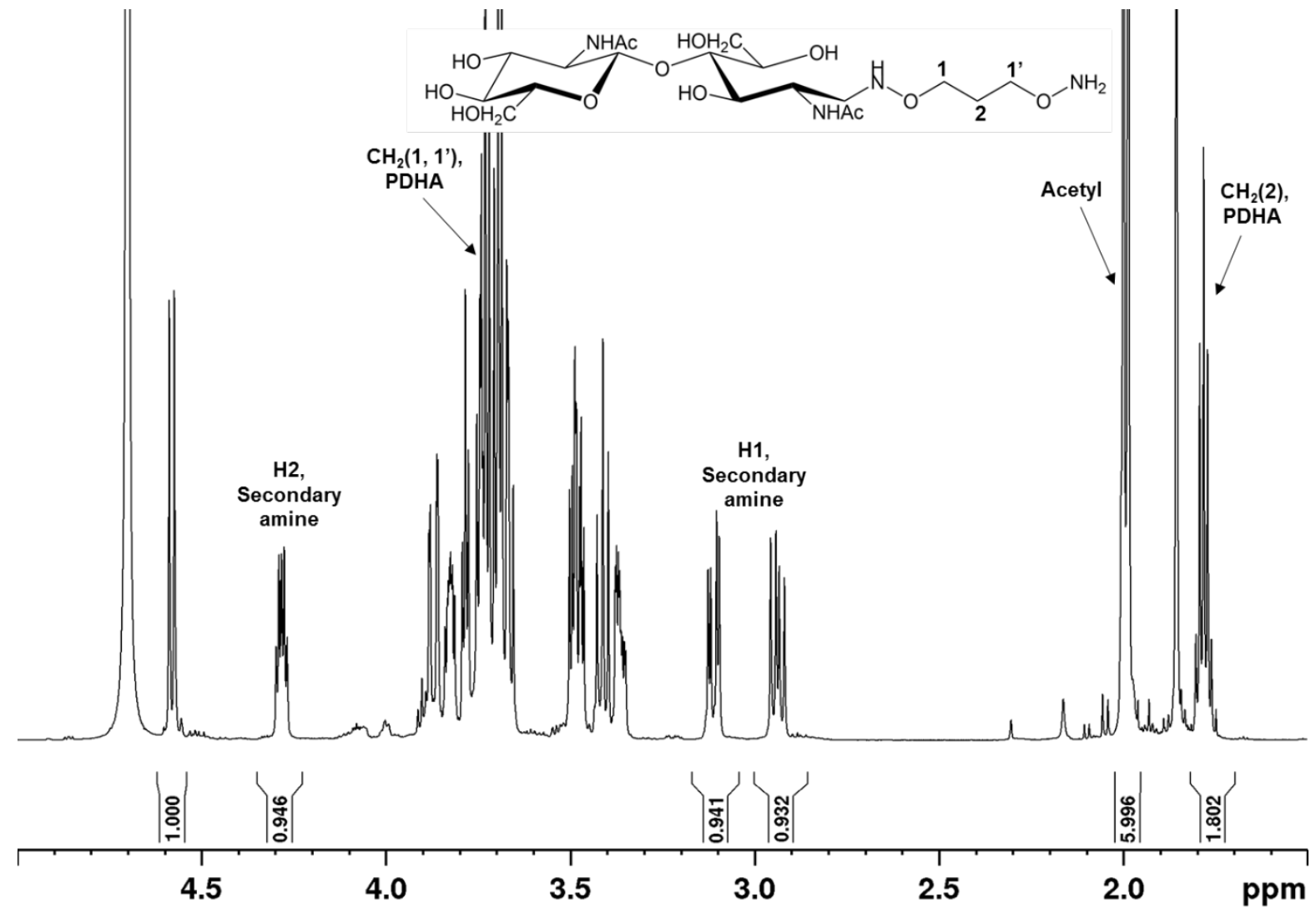

Figure S25: 'H-NMR characterisation of the AA-PDHA fraction from figure S23.

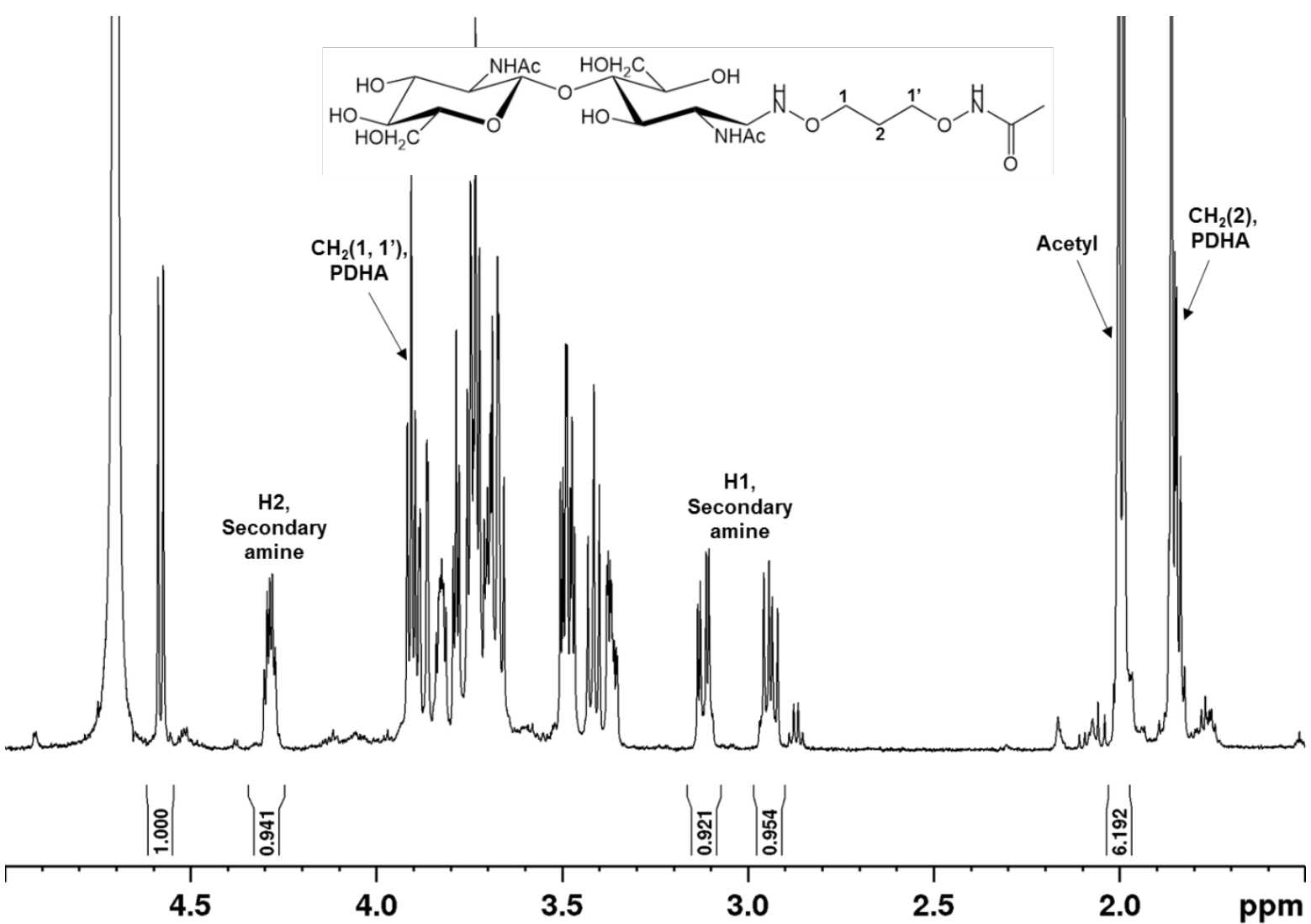

Figure S26: ${ }^{1} \mathrm{H}-\mathrm{NMR}$ characterisation of the AA-PDHA $\left(\mathrm{COCH}_{3}\right)$ fraction from figure S23. 


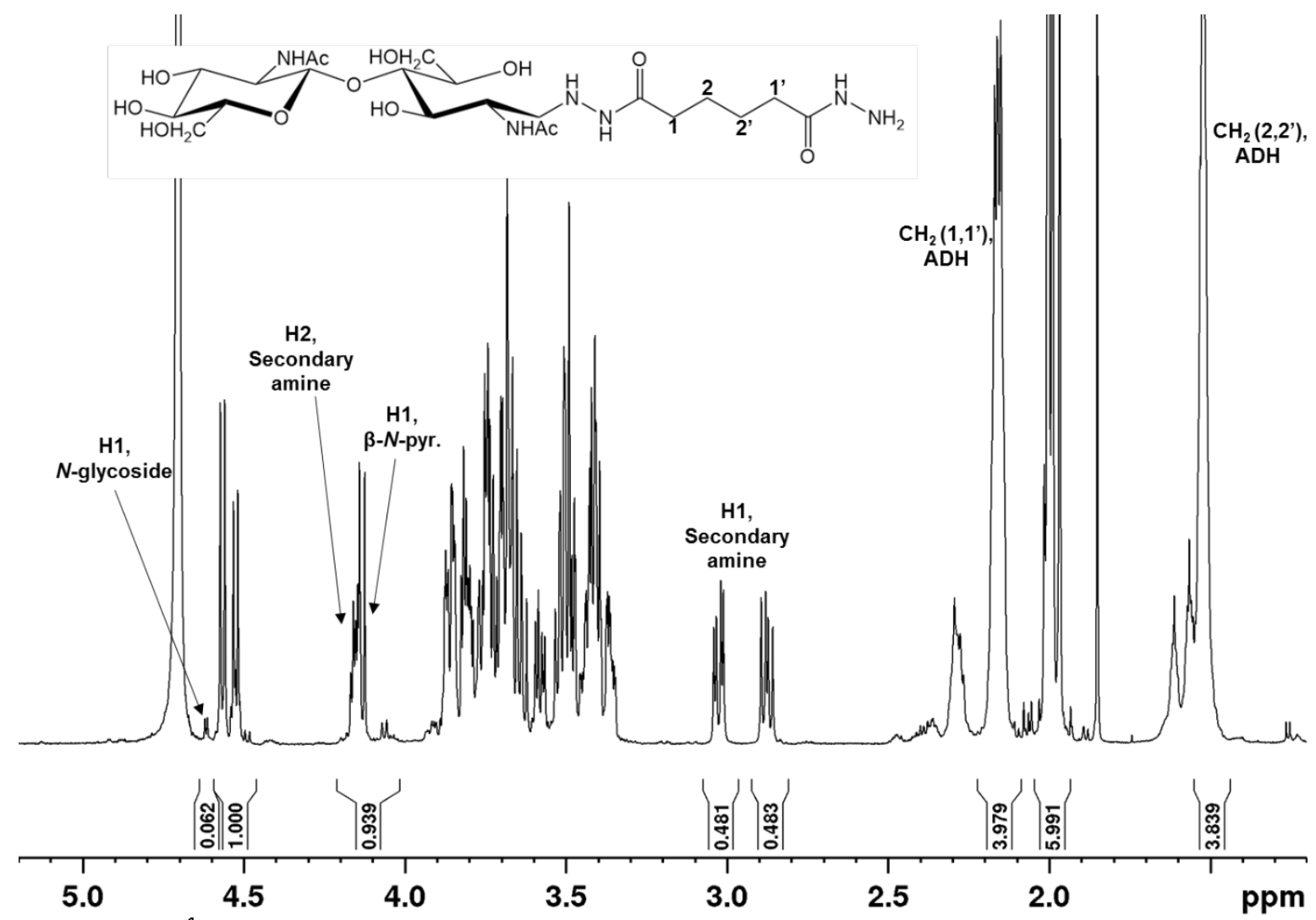

Figure S27: ${ }^{1} \mathrm{H}-\mathrm{NMR}$ characterisation of the AA-ADH fraction from figure S23.

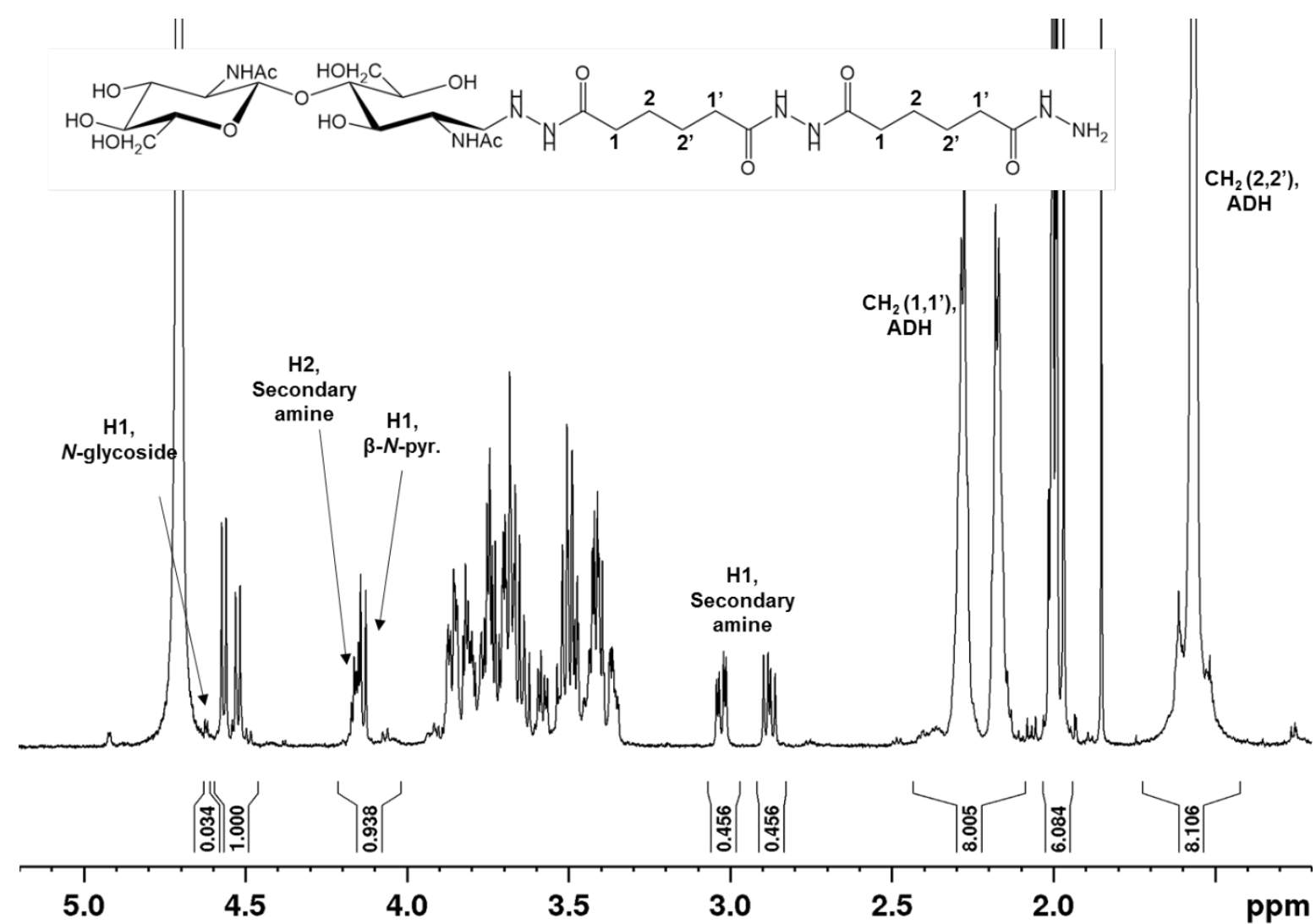

Figure S28: ${ }^{1} \mathrm{H}-\mathrm{NMR}$ characterisation of the $\mathrm{AA}-\mathrm{ADH}_{2}$ fraction from figure S23. The 'polymerization' of ADH is observed by the splitting of the left methylene resonance resulting from the dihydrazide. The right methylene resonance is slightly shifted downfield compared to the AAADH conjugate. 


\section{S12 2D NMR characterisation of the reduced AA-PDHA conjugate}

The main fraction from the reduction of AA-PDHA conjugates (AA-PDHA, Figure S24) was studied in detail by homo- and heteronuclear NMR correlation experiments. The NMR analysis was carried out at the $800 \mathrm{MHz}$ spectrometer in a $3 \mathrm{~mm}$ NMR tube. Resonances were assigned by starting at the anomeric proton resonance and then following the proton-proton connectivity using DQF-COSY in combination with ${ }^{13} \mathrm{C} \mathrm{HSQC}$ and ${ }^{13} \mathrm{C} \mathrm{H} 2 \mathrm{BC}$ spectra. The following designations are used in the spectra displayed in Figure S29 and S30: NRE (the non-reducing end A-unit in the conjugate), RE (the reducing end $A$-unit in the conjugate), \# (unique identified methylene protons from the dioxyamine (PDHA) in the conjugate), $\mathrm{N}$-acetyl ( $\mathrm{N}$-acetyl groups of the A-units in the conjugate). $\mathrm{H \#}$ refers to the proton attached to the ring carbon number $(\mathrm{C \# )}$ for the sugar units. The water resonance (4.75 ppm) was used as chemical shift reference for protons.

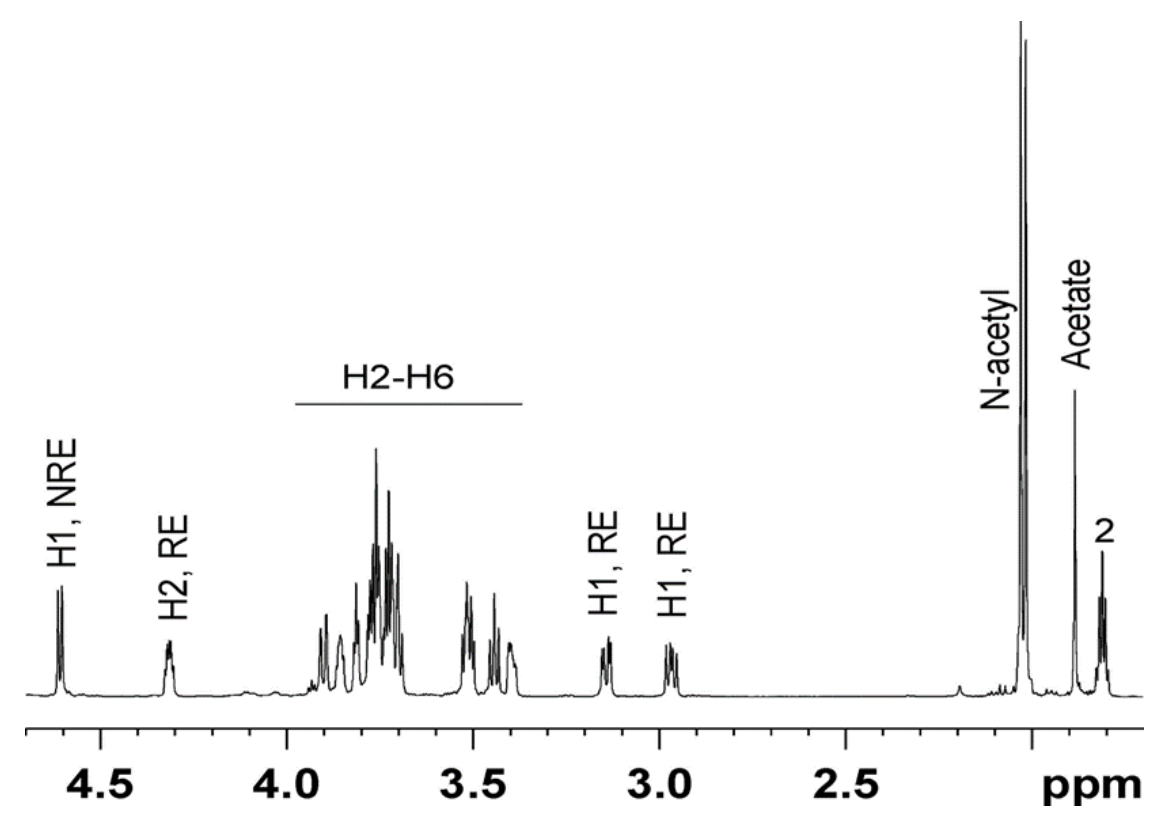

Figure S29: 1D proton spectrum for the reduced form of the AA-PDHA conjugate.

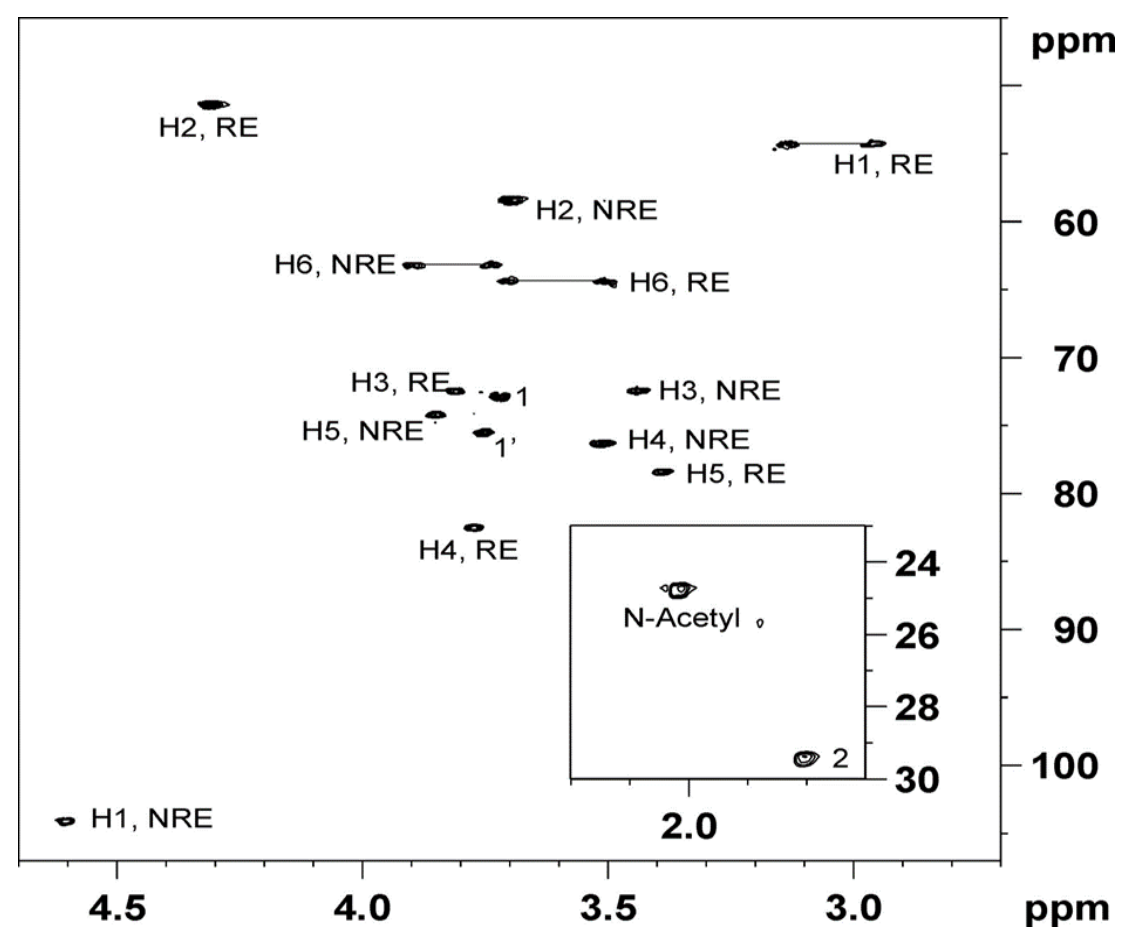


Figure S30: ${ }^{13} \mathrm{C} H S Q C$ spectrum for the reduced form of the AA-PDHA conjugate.

Table S3: Assignment of the chemical shifts for the reduced form of the AA-PDHA conjugate.

\begin{tabular}{|c|c|c|c|c|c|c|c|}
\hline \multirow{2}{*}{$\begin{array}{l}\text { Structural } \\
\text { unit }\end{array}$} & \multicolumn{7}{|c|}{ Assignment } \\
\hline & H-1; C-1 & H-2; C-2 & $\mathrm{H}-3 ; \mathrm{C}-3$ & H-4; C-4 & H-5; C-5 & H-6; C-6 & $\mathrm{Ac}-\mathrm{H} ; \mathrm{C}$ \\
\hline NRE & $4.61 ; 104.0$ & $3.71 ; 58.5$ & $3.44 ; 72.0$ & $3.51 ; 76.3$ & $3.85 ; 74.1$ & $3.75,3.90 ; 63.2$ & $2.01 ; 24.6$ \\
\hline \multirow[t]{2}{*}{ RE } & $2.97 ; 3.14 ; 54.2$ & $4.31 ; 51.4$ & $3.81 ; 72.6$ & $3.76 ; 82.2$ & $3.39 ; 78.2$ & $3.50,3.72 ; 64.3$ & $2.01 ; 24.6$ \\
\hline & \multicolumn{2}{|c|}{$\begin{array}{l}\text { Methylene in PDHA } \\
\text { closest to conjugation }\end{array}$} & \multicolumn{2}{|c|}{$\begin{array}{l}\text { Middle methylene } \\
\text { in PDHA }\end{array}$} & \multicolumn{3}{|c|}{$\begin{array}{l}\text { Methylene in PDHA closest to } \\
\text { free oxyamine end }\end{array}$} \\
\hline PDHA & \multicolumn{2}{|c|}{$4.22 ; 73.1$} & \multicolumn{2}{|c|}{$2.04 ; 29.9$} & \multicolumn{3}{|c|}{$4.14 ; 75.1$} \\
\hline
\end{tabular}

\section{S13 ${ }^{1} \mathrm{H}-\mathrm{NMR}$ characterisation of the reaction mixtures for the reduction of conjugates at higher temperatures}

The mixture of reduced secondary amine conjugates, non-reduced conjugates and unreacted oligomers obtained by one pot reductive amination at higher temperatures was characterised by ${ }^{1} \mathrm{H}$ NMR. The spectra are given in Figure S31-S40. The dimer, AA, was used for the reduction experiments and the yields (\%) were calculated by relating the integrals of the resonances from the various products to the integral of the resonance resulting from the $\mathrm{H} 1$ of the non-reducing A-unit set to $1(100 \%)$ in all spectra. The yield of unidentified products was calculated as the difference between the sum of reduced conjugates, non-reduced conjugates and unreacted oligomers and the theoretical yield $(100 \%)$. As the resonance from $\mathrm{H} 2$ secondary amine completely overlapped the resonance resulting from the $\beta-N$-pyranoside for the AA-ADH conjugates, the remaining non-reduced conjugates was calculated by subtracting the integral resulting from one of the secondary amine protons from the total integral for the overlapping resonances. The following designations are used in the ${ }^{1} \mathrm{H}-\mathrm{NMR}$ spectra: $(E)$ - and $(Z)$-oxime $((E) /(Z)$-configuration of the oxime), $\beta-N$-pyr. ( $\beta$-configuration of the $N$-pyranoside), $N$-glycoside (unidentified $N$-glycoside conjugate), $\alpha$ - and $\beta$ - red. ( $\alpha-\beta-$ configuration of the anomeric proton in the reducing end A-unit) and secondary amine (reduced secondary amine conjugates). 
H1,

$$
\text { (E)-oxime }
$$<smiles>C[18OH]</smiles>

H2,

Secondary amine

H1,

(Z)-oxime

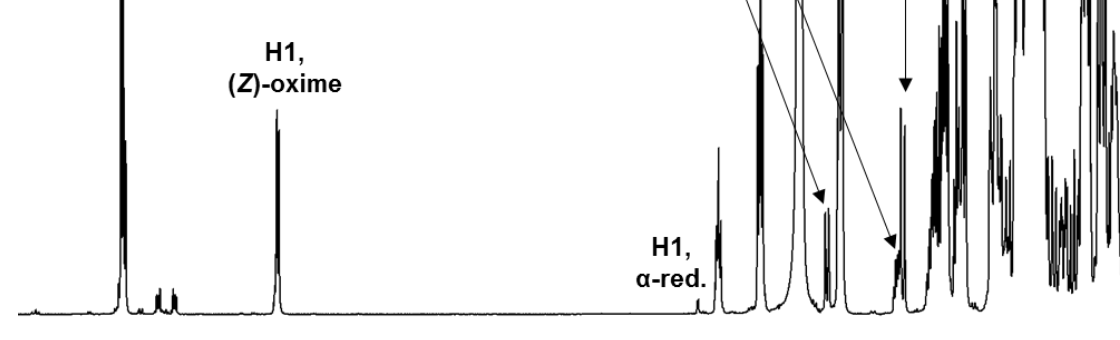

H1,

Secondary amine

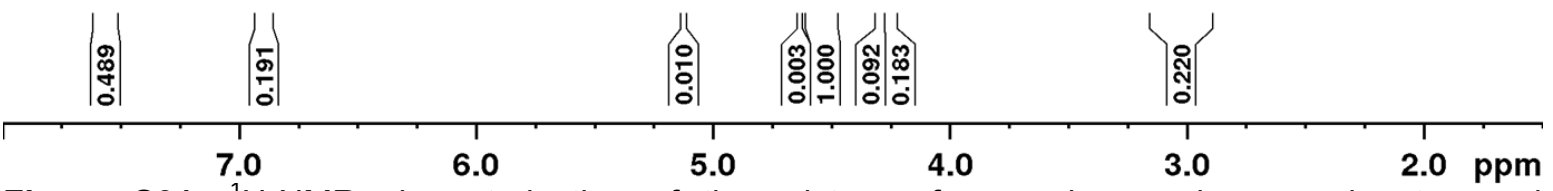

Figure S31: ${ }^{1} \mathrm{H}-\mathrm{NMR}$ characterisation of the mixture of secondary amines, conjugates and unreacted oligomers obtained for the one pot reductive amination with AA, 10 equivalents of PDHA and 20 equivalents of PB at RT for 8 hours.

H1,

$$
\text { (E)-oxime }
$$<smiles>CO[TeH3]</smiles>
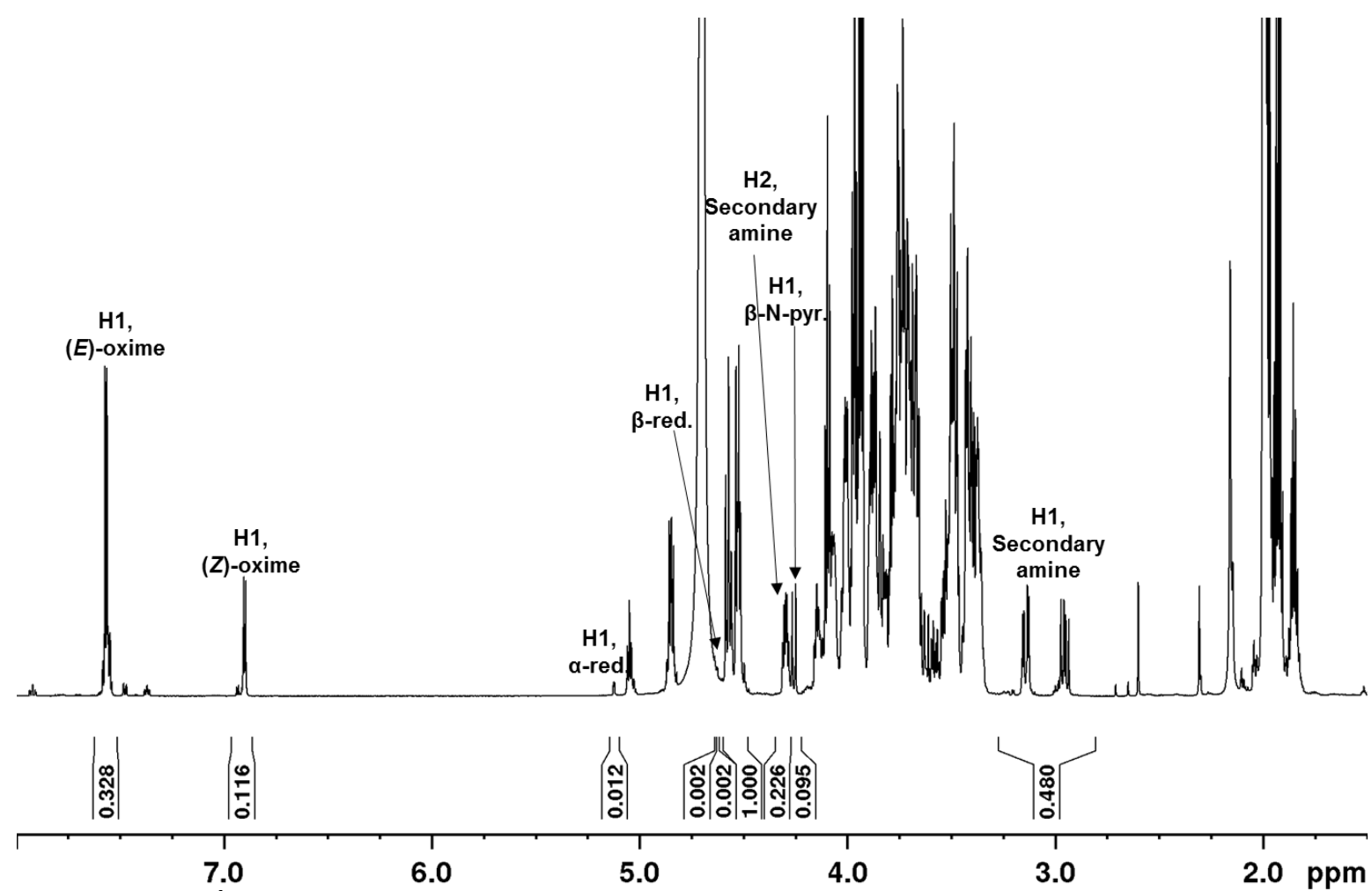

Figure S32: ${ }^{1} \mathrm{H}-\mathrm{NMR}$ characterisation of the mixture of secondary amines, conjugates and unreacted oligomers obtained for the one pot reductive amination with $\mathrm{AA}, 10$ equivalents of PDHA and 20 equivalents of $\mathrm{PB}$ at $40^{\circ} \mathrm{C}$ for 8 hours. 


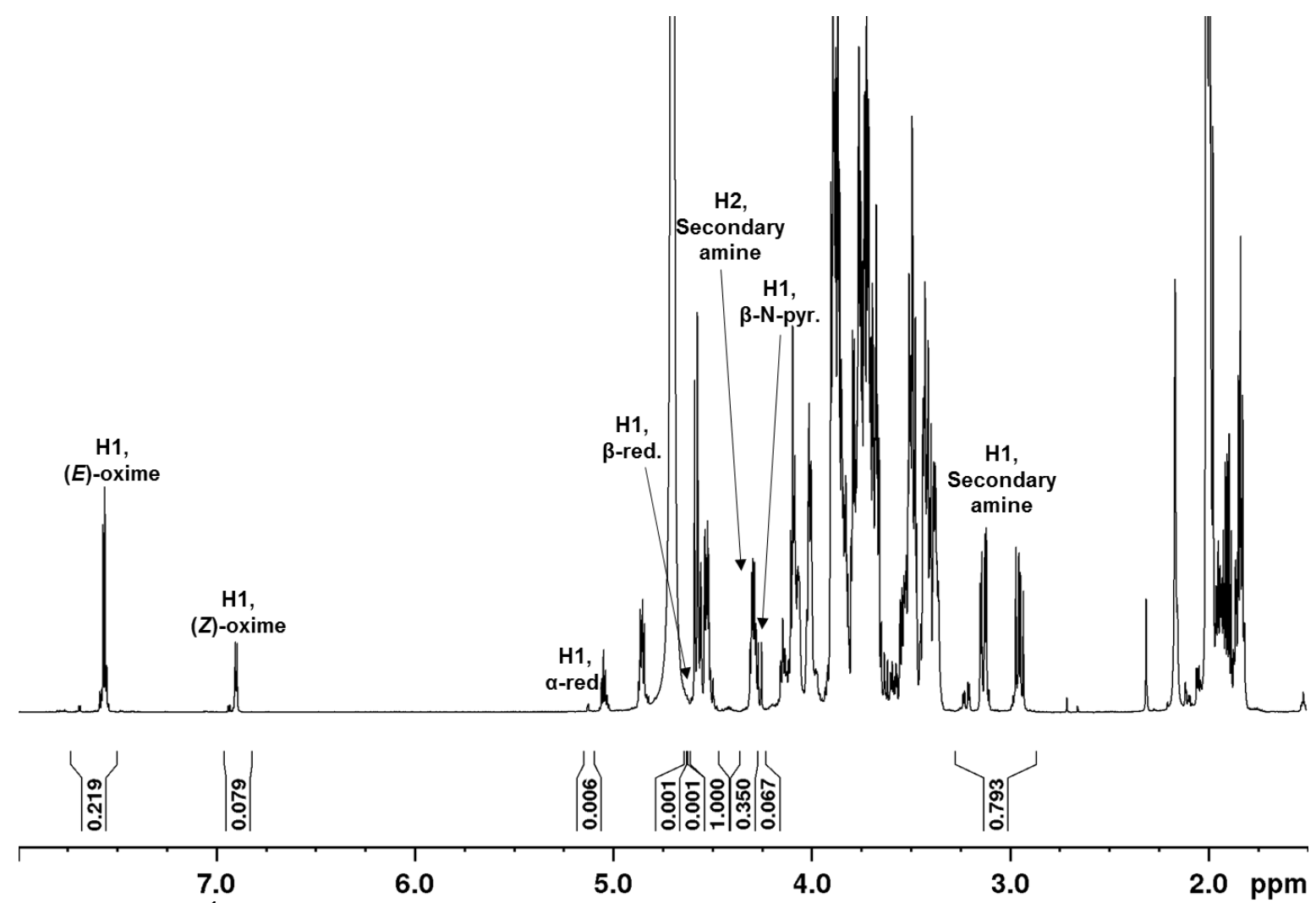

Figure S33: ${ }^{1} \mathrm{H}-\mathrm{NMR}$ characterisation of the mixture of secondary amines, conjugates and unreacted oligomers obtained for the one pot reductive amination with $A A, 10$ equivalents of PDHA and 20 equivalents of $\mathrm{PB}$ at $60^{\circ} \mathrm{C}$ for 8 hours.

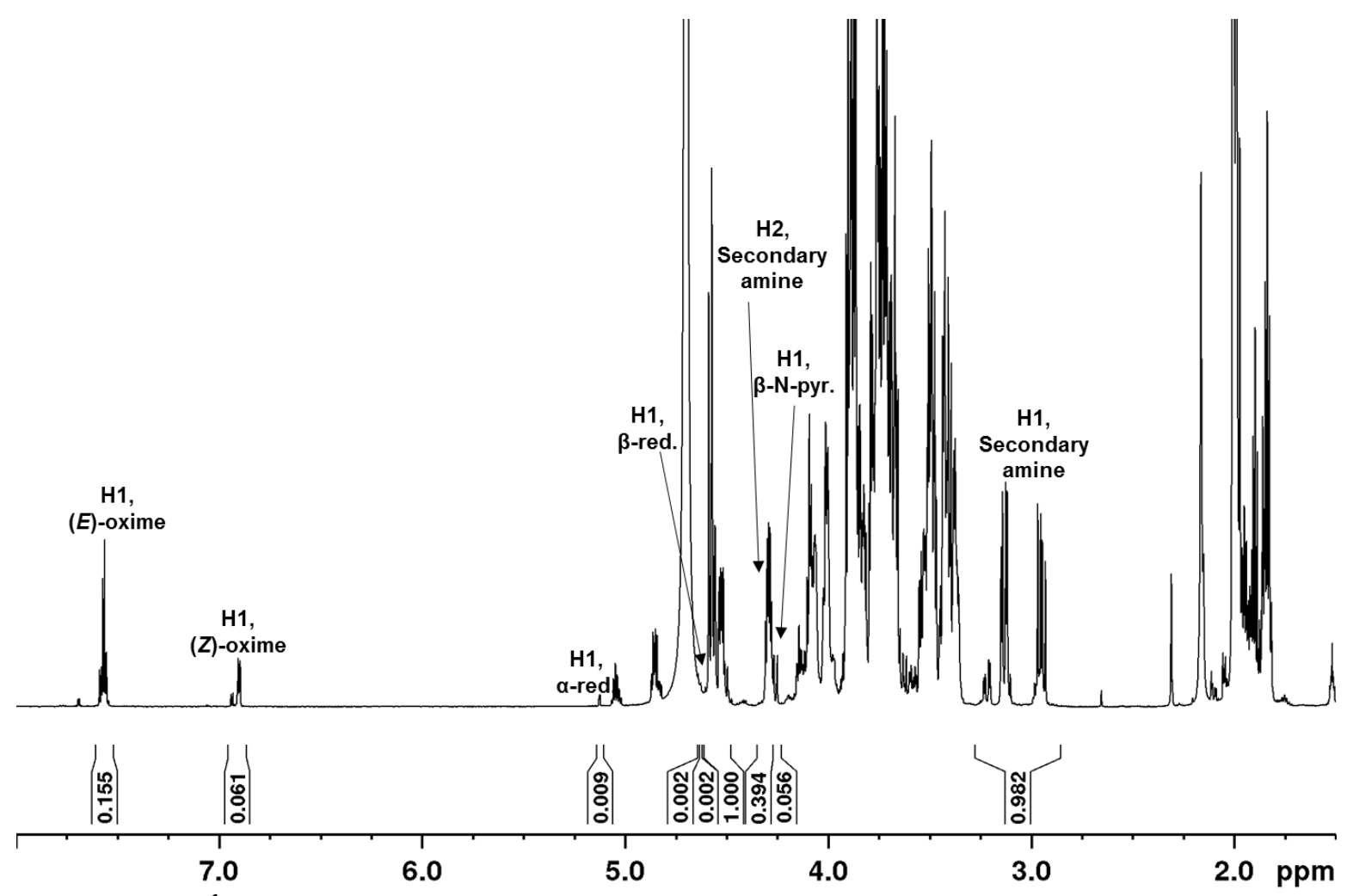

Figure S34: ${ }^{1} \mathrm{H}-\mathrm{NMR}$ characterisation of the mixture of secondary amines, conjugates and unreacted oligomers obtained for the one pot reductive amination with AA, 10 equivalents of PDHA and 20 equivalents of PB added in four portions (5 equivalents added at 0, 2, 4 and 6 hours) at 60 ${ }^{\circ} \mathrm{C}$ for 8 hours. 


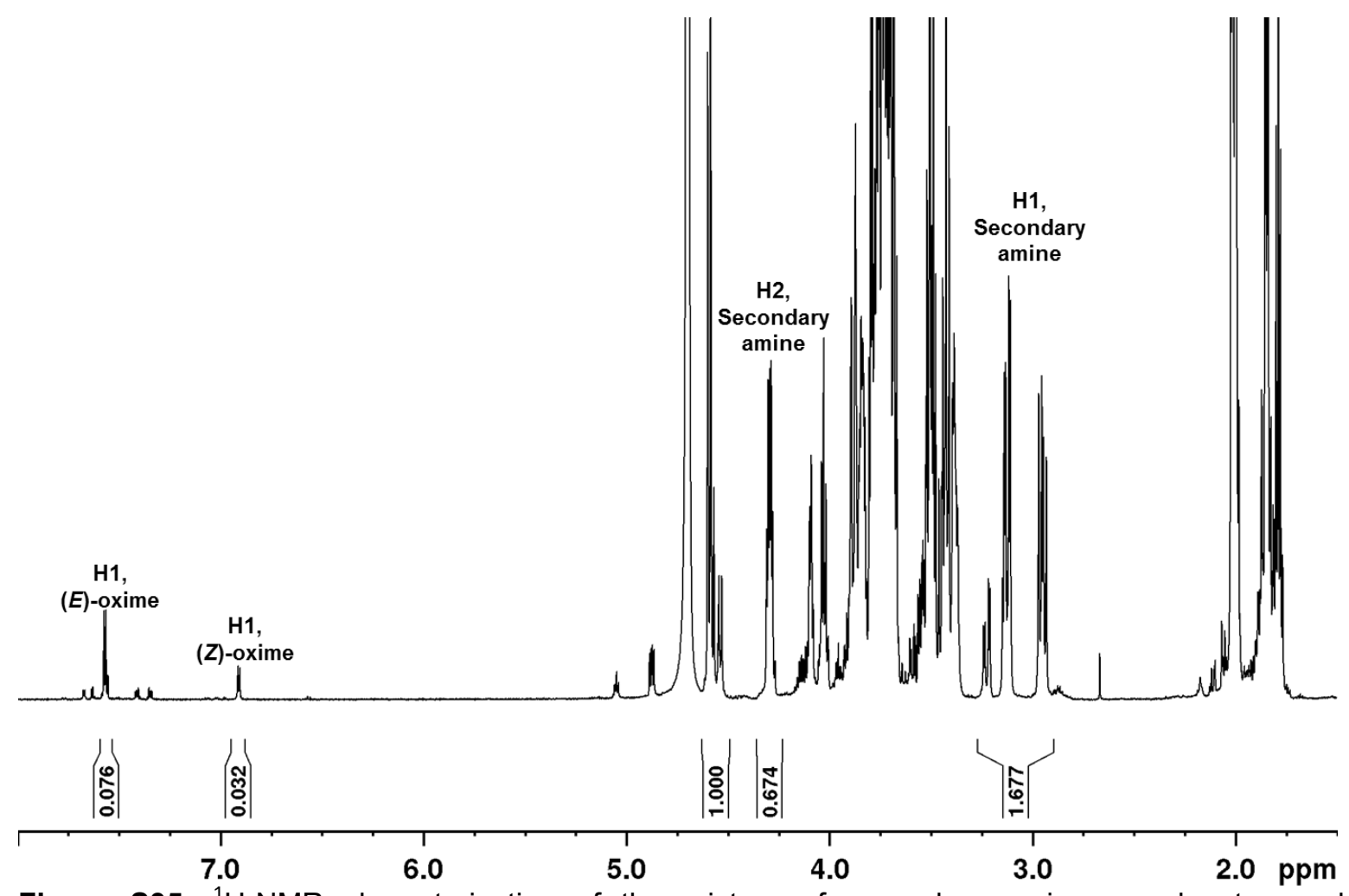

Figure S35: ${ }^{1} \mathrm{H}-\mathrm{NMR}$ characterisation of the mixture of secondary amines, conjugates and unreacted oligomers obtained for the one pot reductive amination with AA, 10 equivalents of PDHA and 40 equivalents of PB added in five portions (5 equivalents added at $0,2,4$ and 6 hours and 20 equivalents added after 8 hours) at $60^{\circ} \mathrm{C}$ for 24 hours.

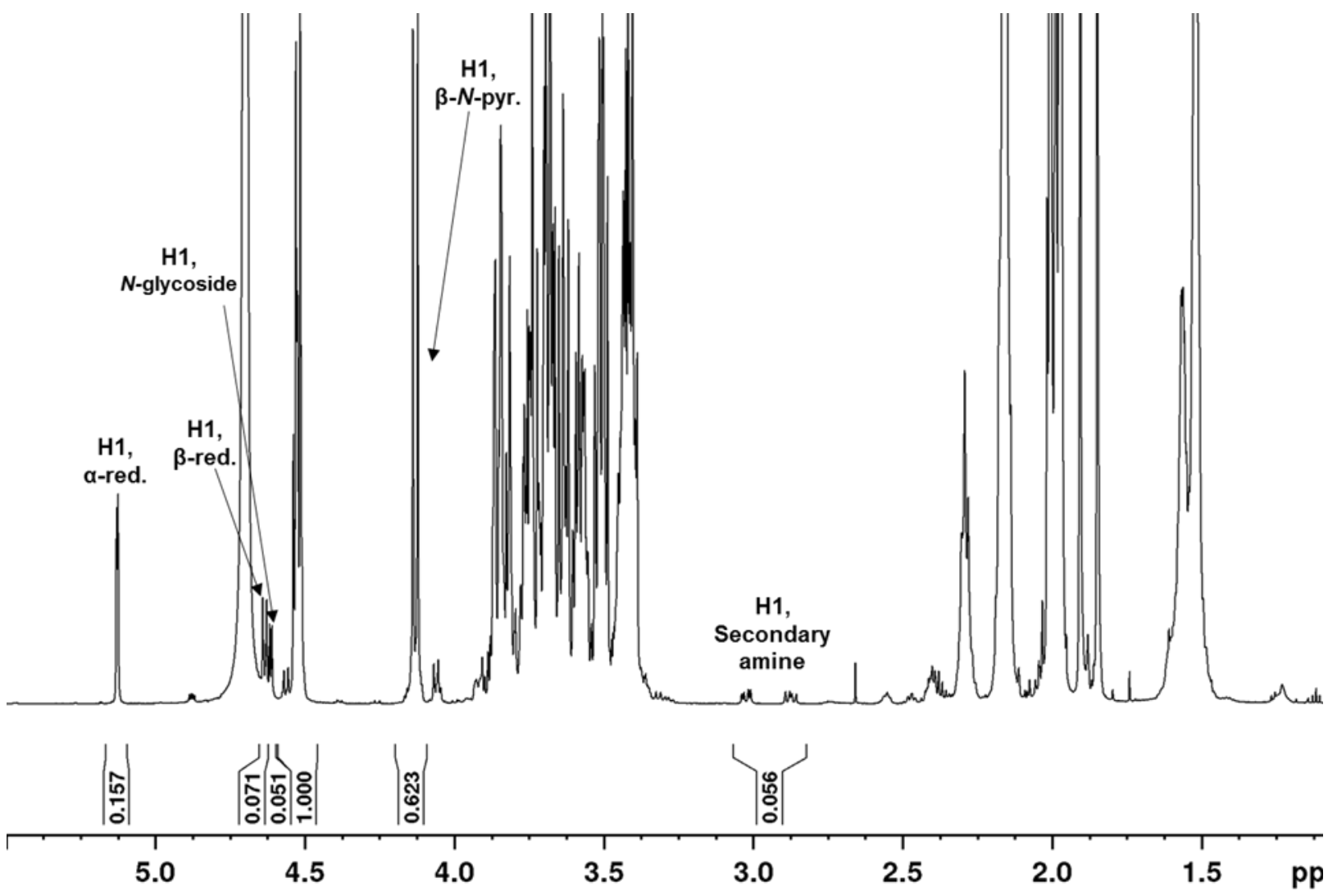

Figure S36: ${ }^{1} \mathrm{H}-\mathrm{NMR}$ characterisation of the mixture of secondary amines, conjugates and unreacted oligomers obtained for the one pot reductive amination with AA, 10 equivalents of ADH and 20 equivalents of PB at RT for 8 hours. 


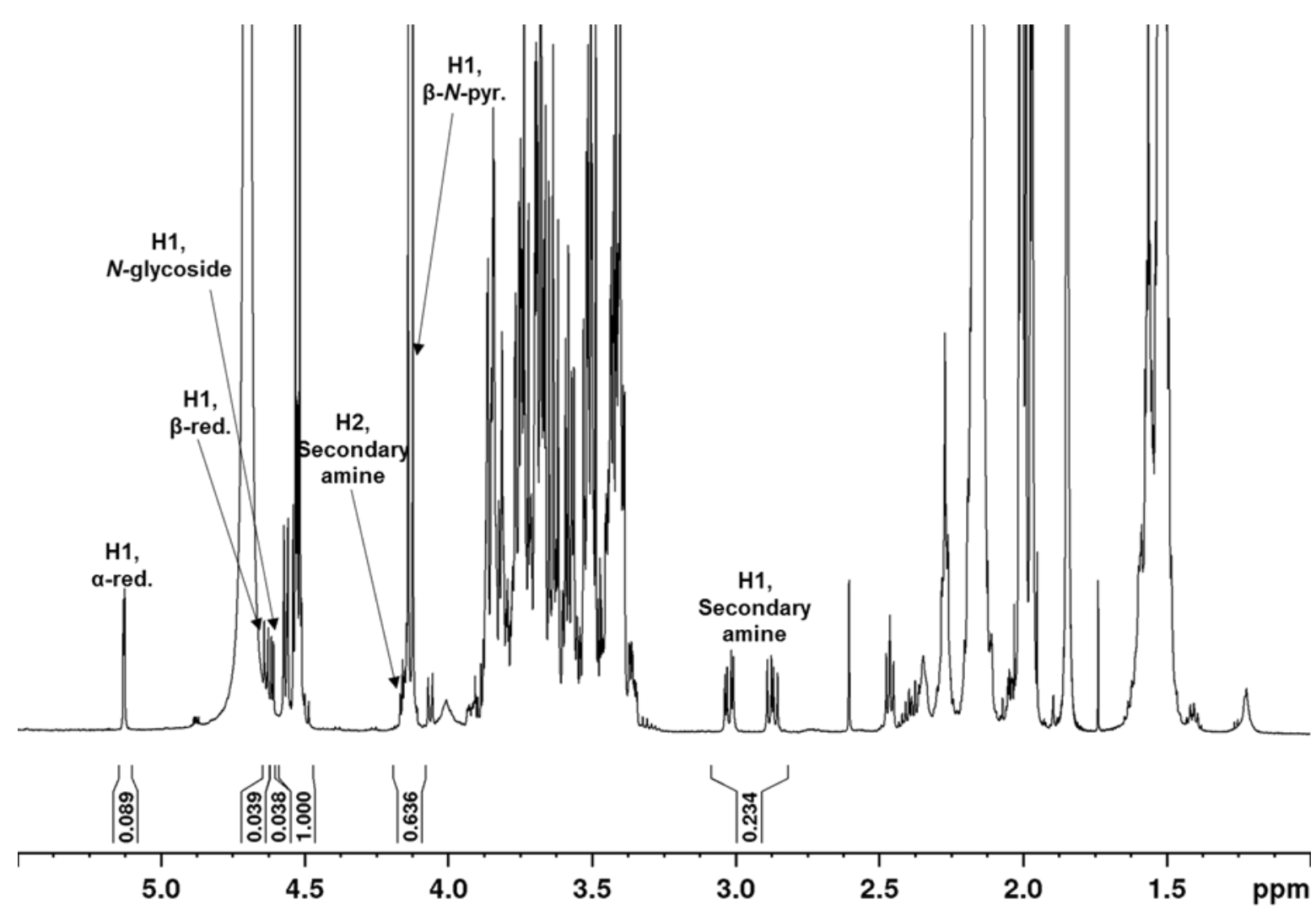

Figure S37: ${ }^{1} \mathrm{H}-\mathrm{NMR}$ characterisation of the mixture of secondary amines, conjugates and unreacted oligomers obtained for the one pot reductive amination with $A A, 10$ equivalents of $A D H$ and 20 equivalents of $\mathrm{PB}$ at $40^{\circ} \mathrm{C}$ for 8 hours.

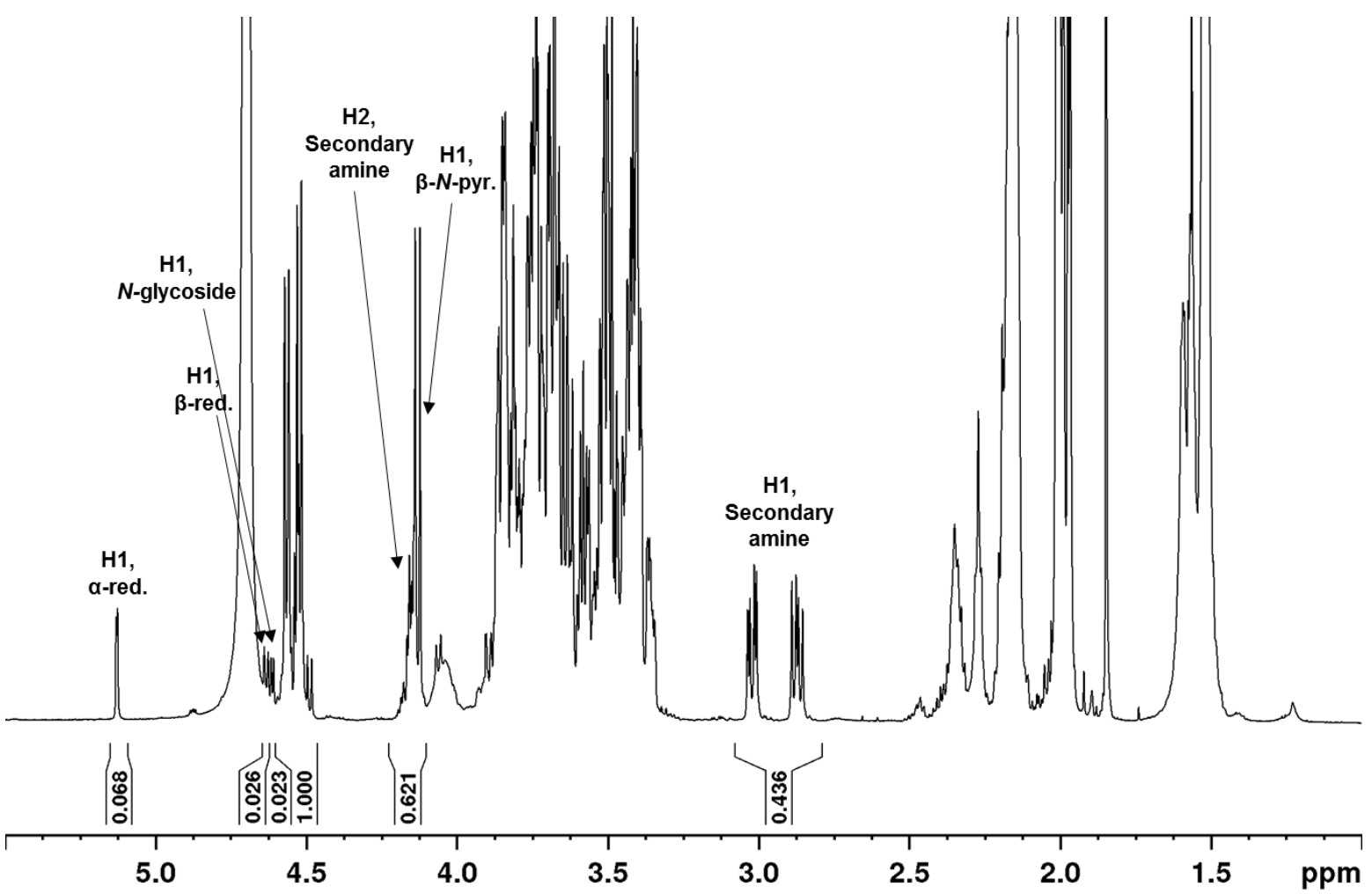

Figure S38: ${ }^{1} \mathrm{H}-\mathrm{NMR}$ characterisation of the mixture of secondary amines, conjugates and unreacted oligomers obtained for the one pot reductive amination with AA, 10 equivalents of ADH and 20 equivalents of $\mathrm{PB}$ at $60^{\circ} \mathrm{C}$ for 8 hours. 


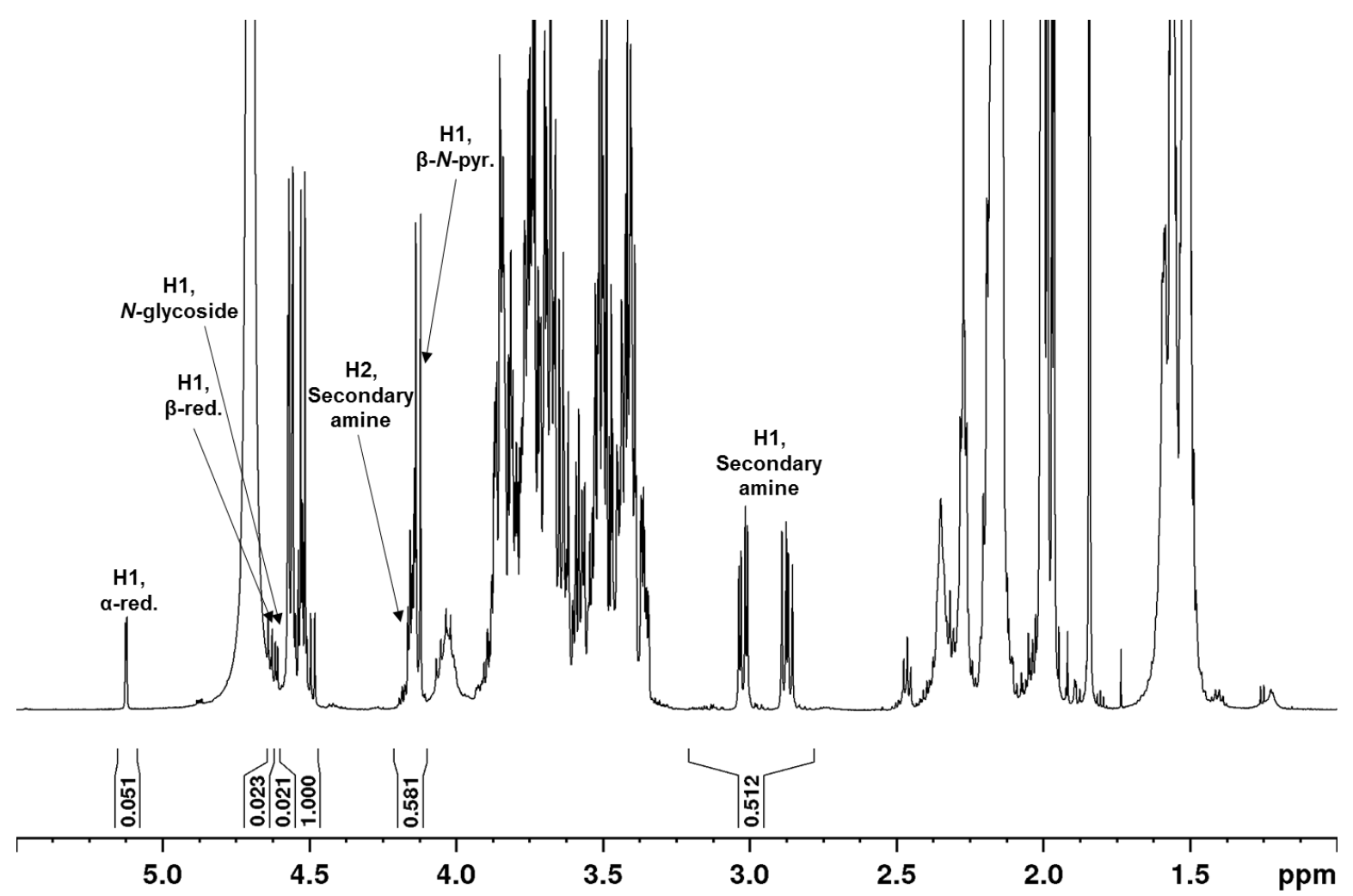

Figure S39: ${ }^{1} \mathrm{H}$-NMR characterisation of the mixture of secondary amines, conjugates and unreacted oligomers obtained for the one pot reductive amination with AA, 10 equivalents of ADH and 20 equivalents of PB added in four portions (5 equivalents added at $0,2,4$ and 6 hours) at 60 ${ }^{\circ} \mathrm{C}$ for 8 hours.

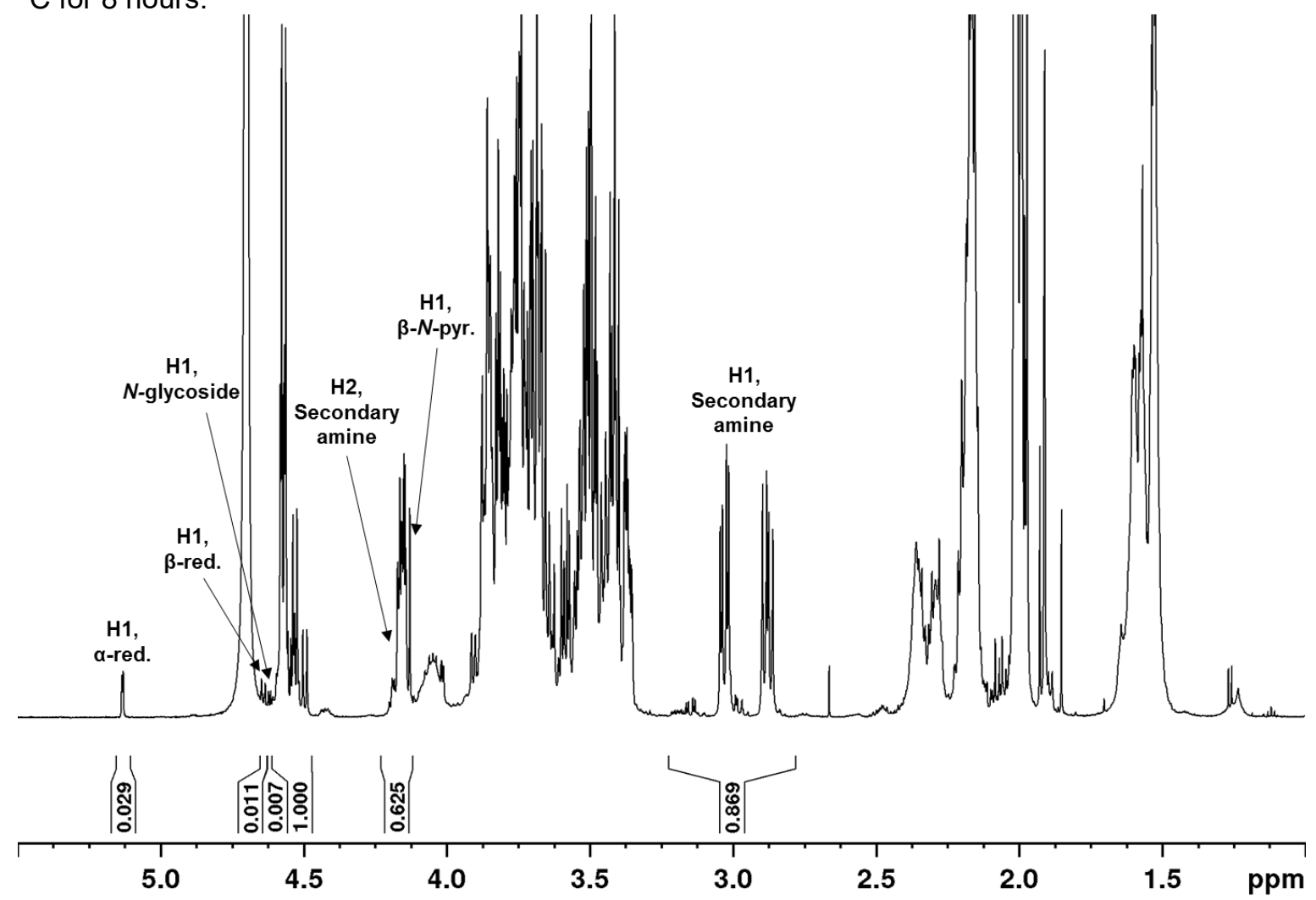

Figure S40: ${ }^{7} \mathrm{H}$-NMR characterisation of the mixture of secondary amines, conjugates and unreacted oligomers obtained for the one pot reductive amination with AA, 10 equivalents of ADH and 40 equivalents of PB added in five portions (5 equivalents added at $0,2,4$ and 6 hours and 20 equivalents added after 8 hours) at $60^{\circ} \mathrm{C}$ for 24 hours. 


\section{S14 Conjugation of PDHA and ADH to Dext $_{1}(\mathrm{Glc})$ studied by time course NMR}

Conjugation of $\mathrm{ADH}$ and PDHA to Dext ${ }_{1}(\mathrm{Glc})$ was studied by time course NMR. ${ }^{1} \mathrm{H}-\mathrm{NMR}$ spectra at defined time points and the course of the reaction using 2 equivalents of PDHA and ADH at pH 4.0, are given in Figure S41 and S42, respectively. Yields (\%) were calculated by relating the integrals (not included) of the resonance resulting from the $\mathrm{H} 1$ reducing end unit for the conjugates and unreacted oligomers at specific time points to the sum of these integrals (100\%, theoretical yield).

a)

$$
\text { Glc }
$$

b)

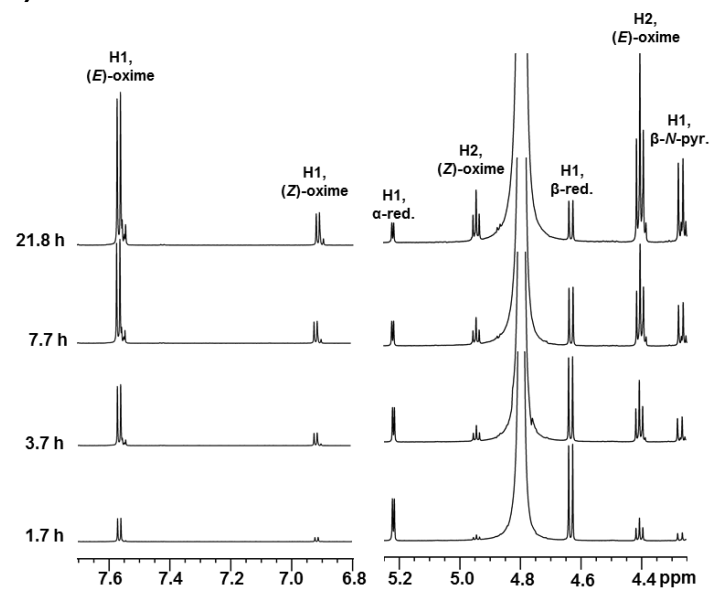

c)

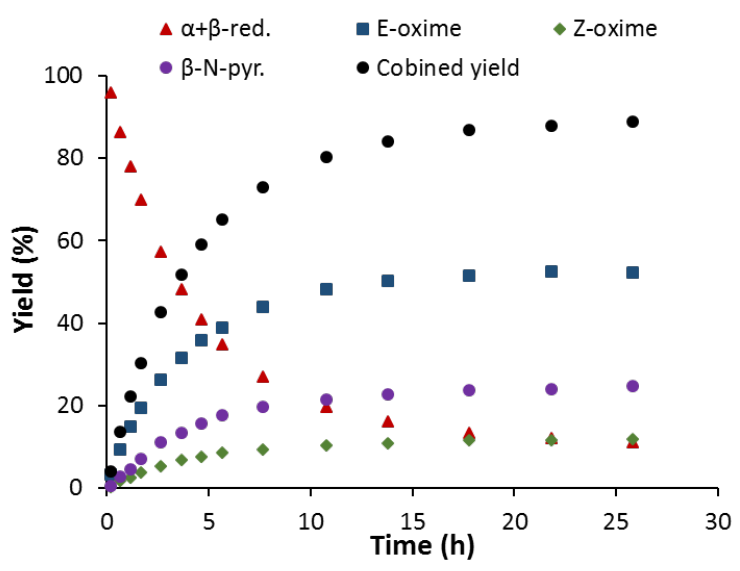

Figure S41: a) conjugation of PDHA to $\operatorname{Dext}_{1}$ (Glc), b) ${ }^{1} \mathrm{H}-\mathrm{NMR}$ spectra at defined time points for the conjugation of PDHA (2 equivalents) to Dext $\mathrm{D}_{1}(\mathrm{Glc}), \mathrm{pH} 4.0 \mathrm{c}$ ) course of the reaction obtained from the integration of the spectra shown in a).

a)

b)<smiles>NNC(=O)CCCCC(=O)NNNC(=O)O</smiles>

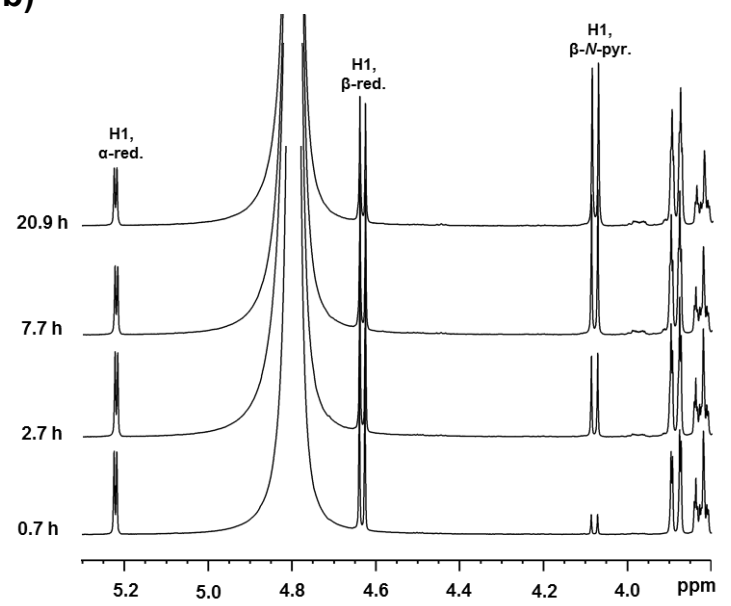

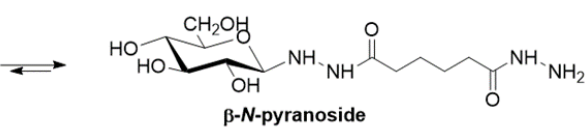

c)

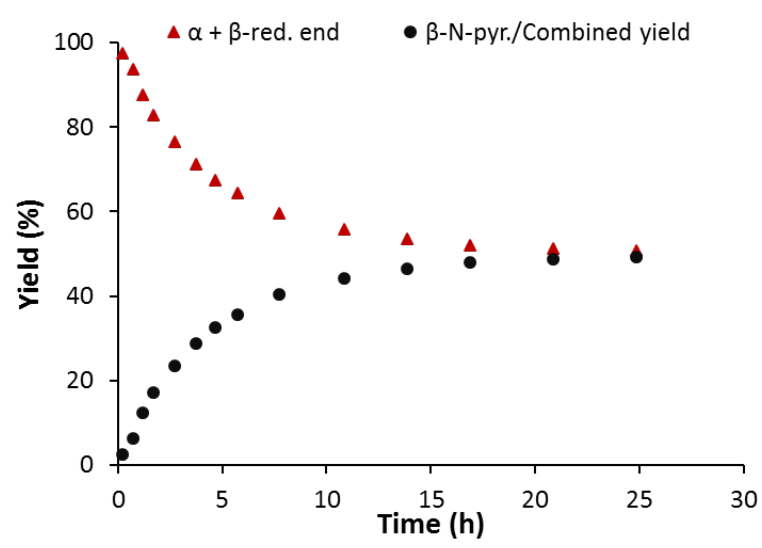

Figure S42: a) conjugation of $\mathrm{ADH}$ to $\operatorname{Dext}_{1}(\mathrm{Glc})$, b) ${ }^{1} \mathrm{H}-\mathrm{NMR}$ spectra at defined time points for the conjugation of $\mathrm{ADH}$ (2 equivalents) to $\operatorname{Dext}_{1}(\mathrm{Glc}), \mathrm{pH} 4.0 \mathrm{c}$ ) course of the reaction obtained from the integration of the spectra shown in a). 
S15 ${ }^{1} \mathrm{H}-\mathrm{NMR}$ characterisation of the equilibrium mixtures for the conjugation reactions with Dext $_{\mathrm{n}}$ oligomers

The mixture of conjugates and unreacted oligomers at equilibrium for the reactions of Dext $\mathrm{n}_{n}$ with 2 equivalents PDHA or ADH at $\mathrm{pH} 4.0$ were characterized by ${ }^{1} \mathrm{H}-\mathrm{NMR}$. The spectra are given in Figure S43-S46. The following designations are used in the ${ }^{1} \mathrm{H}-\mathrm{NMR}$ spectra: $(E)$ - and $(Z)$-oxime $((E) /(Z)-$ configuration of the oxime), $\beta-N$-pyr. ( $\beta$-configuration of the $N$-pyranoside) and $\alpha$ - and $\beta$-red. ( $\alpha-/ \beta$ configuration of the anomeric proton in the reducing end Glc-unit).

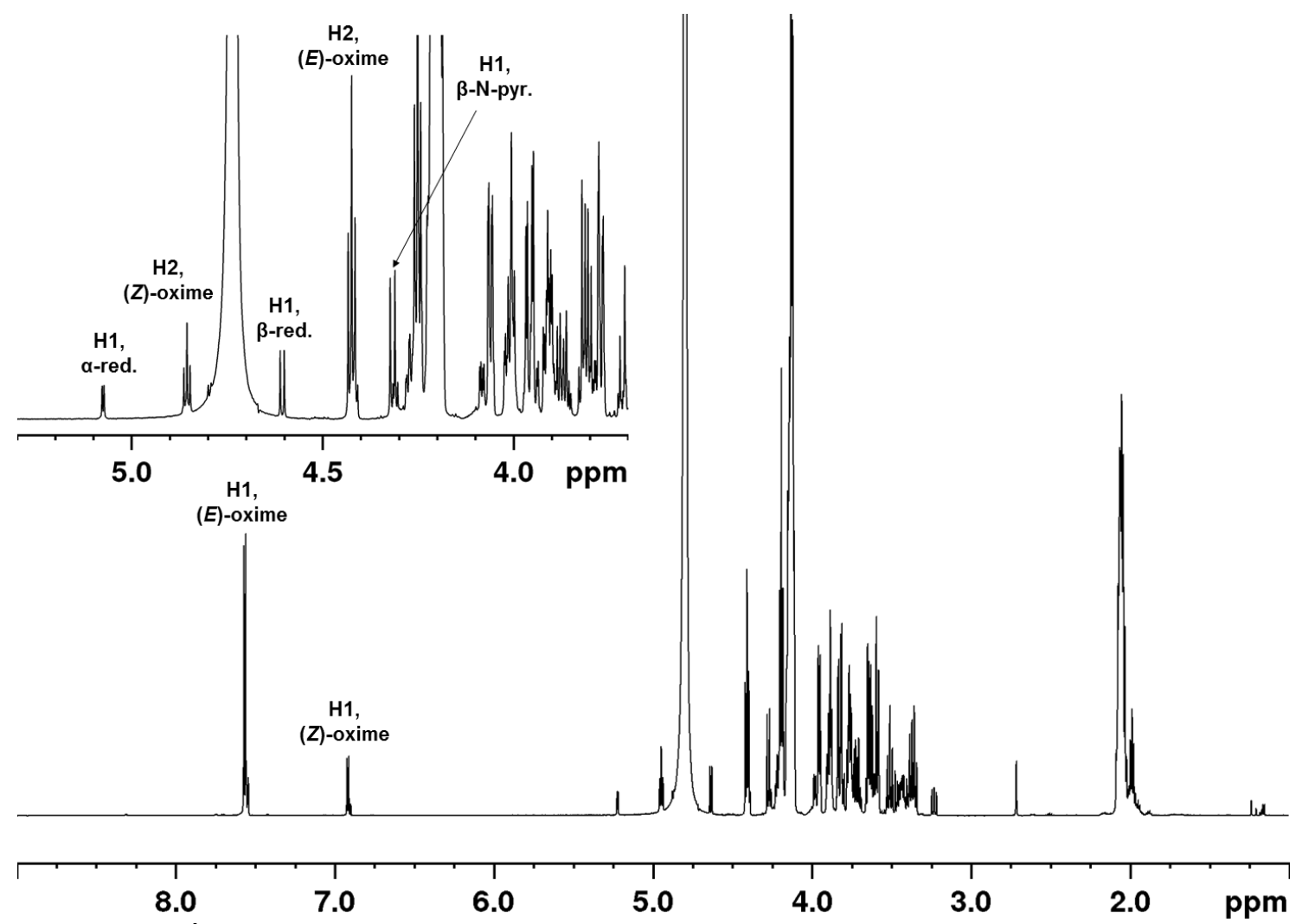

Figure S43: ${ }^{1} \mathrm{H}-\mathrm{NMR}$ characterisation of the mixture of conjugates and unreacted oligomers at equilibrium for the reaction of $\operatorname{Dext}_{1}$ (Glc) with 2 equivalents of PDHA in deuterated NaAc-buffer, $\mathrm{pH}$ 4.0. The yield of conjugates at equilibrium was $92 \%$. 


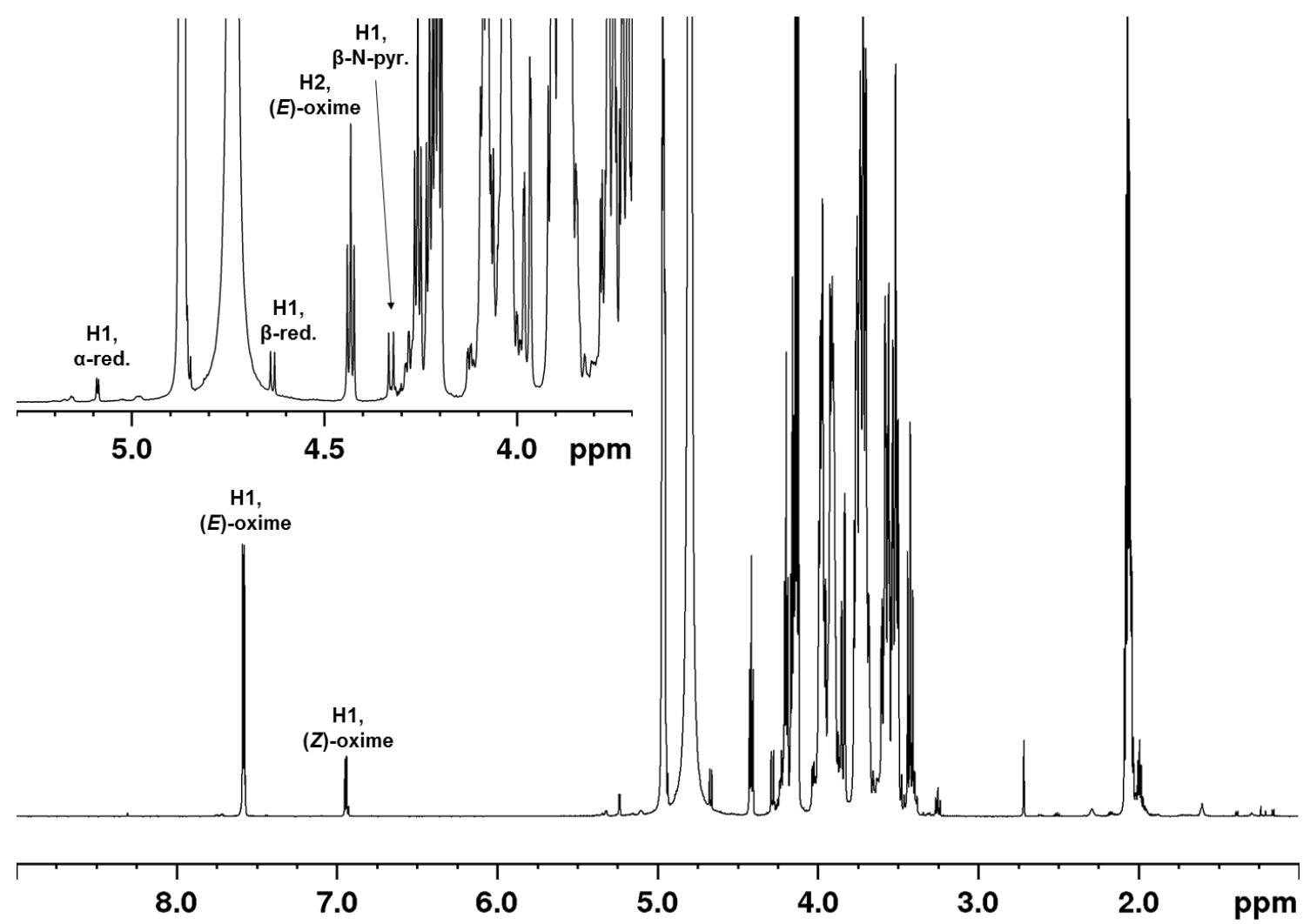

Figure S44: ${ }^{1} \mathrm{H}-\mathrm{NMR}$ characterisation of the mixture of conjugates and unreacted oligomers at equilibrium for the reaction of Dext ${ }_{5}$ with 2 equivalents of PDHA in deuterated NaAc-buffer, $\mathrm{pH}$ 4.0. The yield of conjugates at equilibrium was $90 \%$.
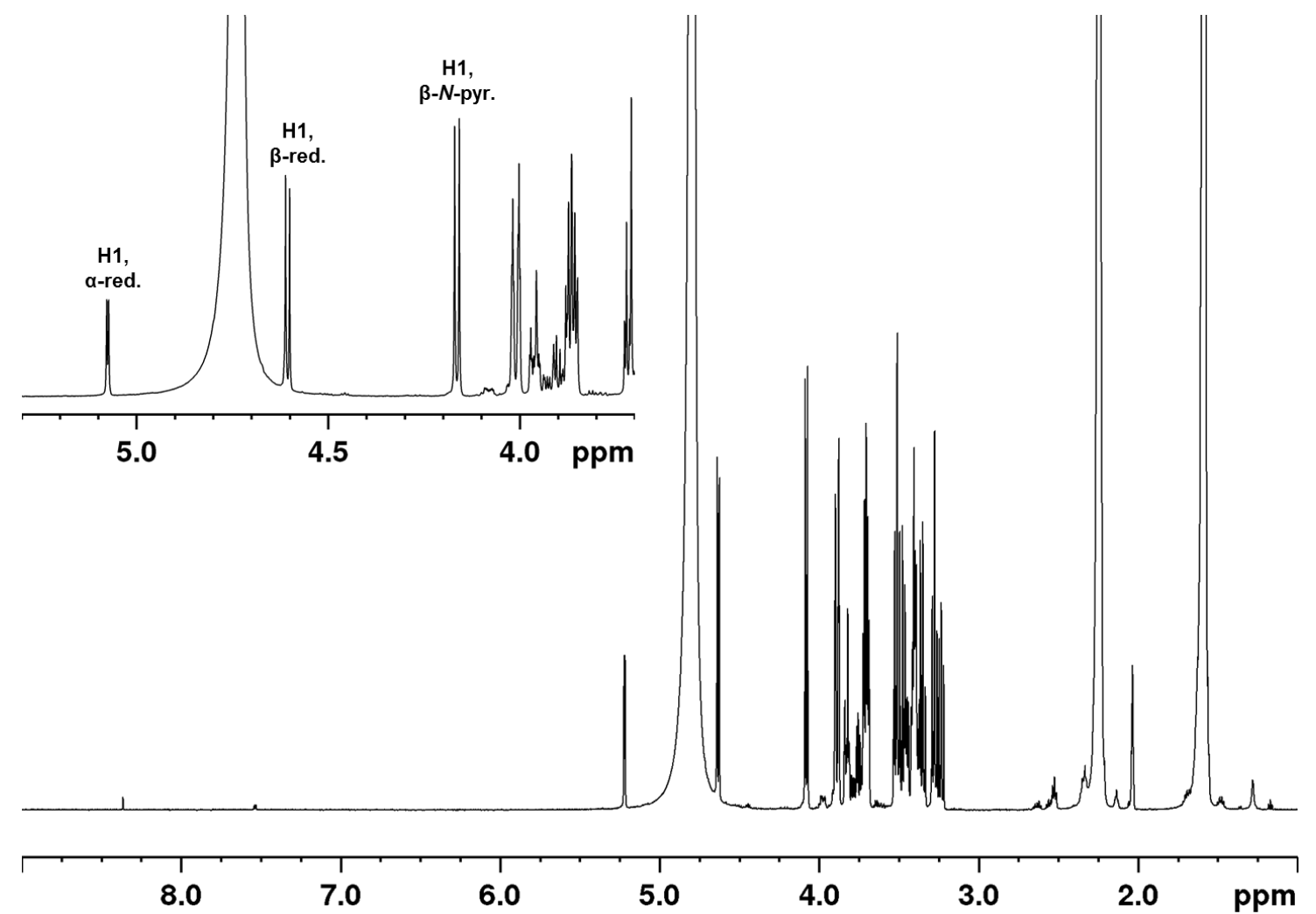

Figure S45: ${ }^{1} \mathrm{H}-\mathrm{NMR}$ characterisation of the mixture of conjugates and unreacted oligomers at equilibrium for the reaction of Dext ${ }_{1}(\mathrm{Glc})$ with 2 equivalents of $\mathrm{ADH}$ in deuterated $\mathrm{NaAc}$-buffer, $\mathrm{pH}$ 4.0. The yield of conjugates at equilibrium was $49 \%$. 


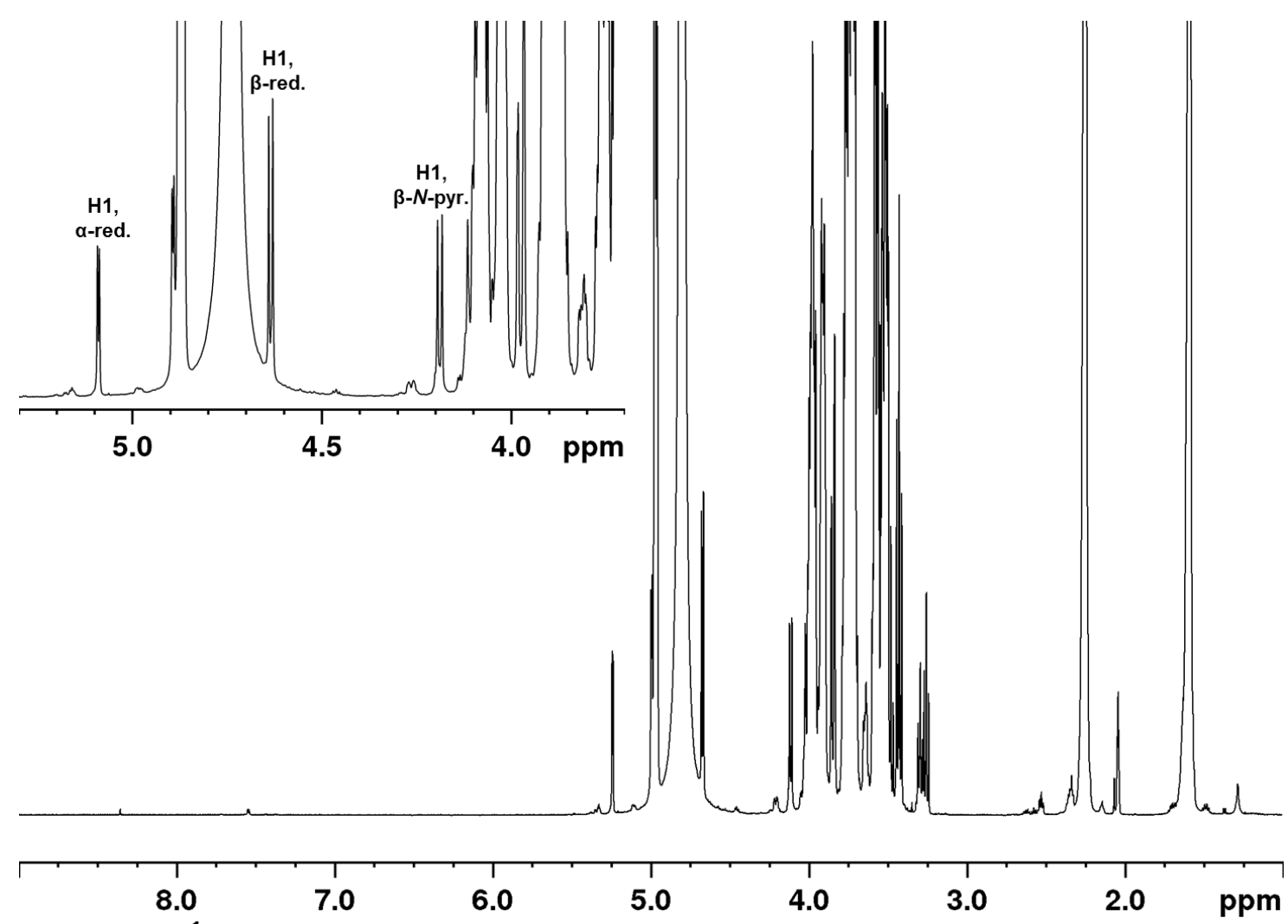

Figure S46: ${ }^{1} \mathrm{H}-\mathrm{NMR}$ characterisation of the mixture of conjugates and unreacted oligomers at equilibrium for the reaction of Dext ${ }_{5}$ with 2 equivalents of $\mathrm{ADH}$ in deuterated NaAc-buffer, pH 4.0. The yield of conjugates at equilibrium was $38 \%$.

\section{S16 ${ }^{1} \mathrm{H}-\mathrm{NMR}$ characterisation of the reaction mixtures for the one pot reductive amination reaction of AA and Dext 4 with PDHA or ADH after 2 and 5 days}

The one pot reductive amination of $\mathrm{AA}$ and Dext $_{4}$ with 2 equivalents of $\mathrm{ADH}$ or PDHA and 3 equivalents of $\mathrm{PB}$ at $\mathrm{pH} 4.0$ was studied by time course NMR. The spectra obtained for the mixture of reduced secondary amine conjugates, non-reduced conjugates (oximes/hydrazones and $\mathrm{N}$ glycosides) and unreacted oligomers after 2 and 5 are given in Figure S47-S50. The following designations are used in the ${ }^{1} \mathrm{H}$-NMR spectra: $(E)$ - and $(Z)$-oxime $((E) /(Z)$-configuration of the oxime), $\beta$ - $N$-pyr. ( $\beta$-configuration of the $N$-pyranoside), $N$-glycoside (unidentified $N$-glycoside conjugate), $\alpha-$ and $\beta$ - red. ( $\alpha-/ \beta$-configuration of the anomeric protons in the reducing end unit of the unreacted oligomers) and secondary amine (reduced secondary amine conjugates). In addition, resonances resulting from PB both in its reduced ( $\mathrm{r}$ ) and oxidized form (in the area 7.3 to $8.7 \mathrm{ppm}$ ) are assigned in the spectra. Yields (\%) were calculated by relating the integrals (not included) of the resonances resulting from the $\mathrm{H} 1$ reducing end unit for the secondary amines, conjugates and unreacted oligomers the sum of these integrals at the start of the reduction (100\%, theoretical yield). The yield of unidentified products was calculated as the difference between the obtained and theoretical yield. The yields (\%) obtained after 2 and 5 days of reaction are summarized in Table S4. 


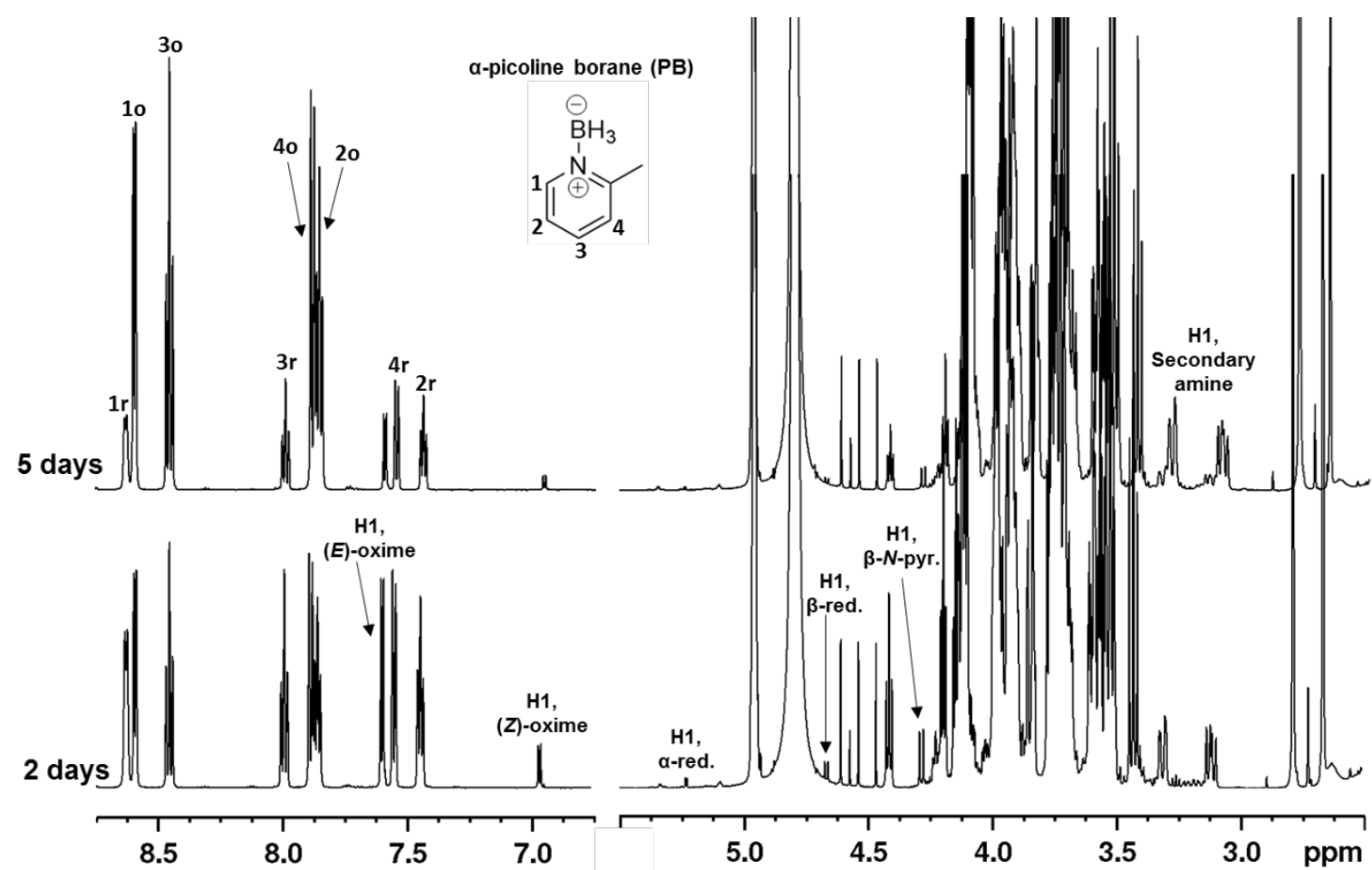

Figure S47: ${ }^{1} \mathrm{H}-\mathrm{NMR}$ characterisation of the mixture of secondary amines, conjugates and unreacted oligomers obtained after 2 and 5 days for the one pot reductive amination of Dext ${ }_{4}$ with 2 equivalents PDHA and 3 equivalents $\mathrm{PB}$ at $\mathrm{pH}$ 4.0. The yield of secondary amines obtained after 5 days was $71 \%$.

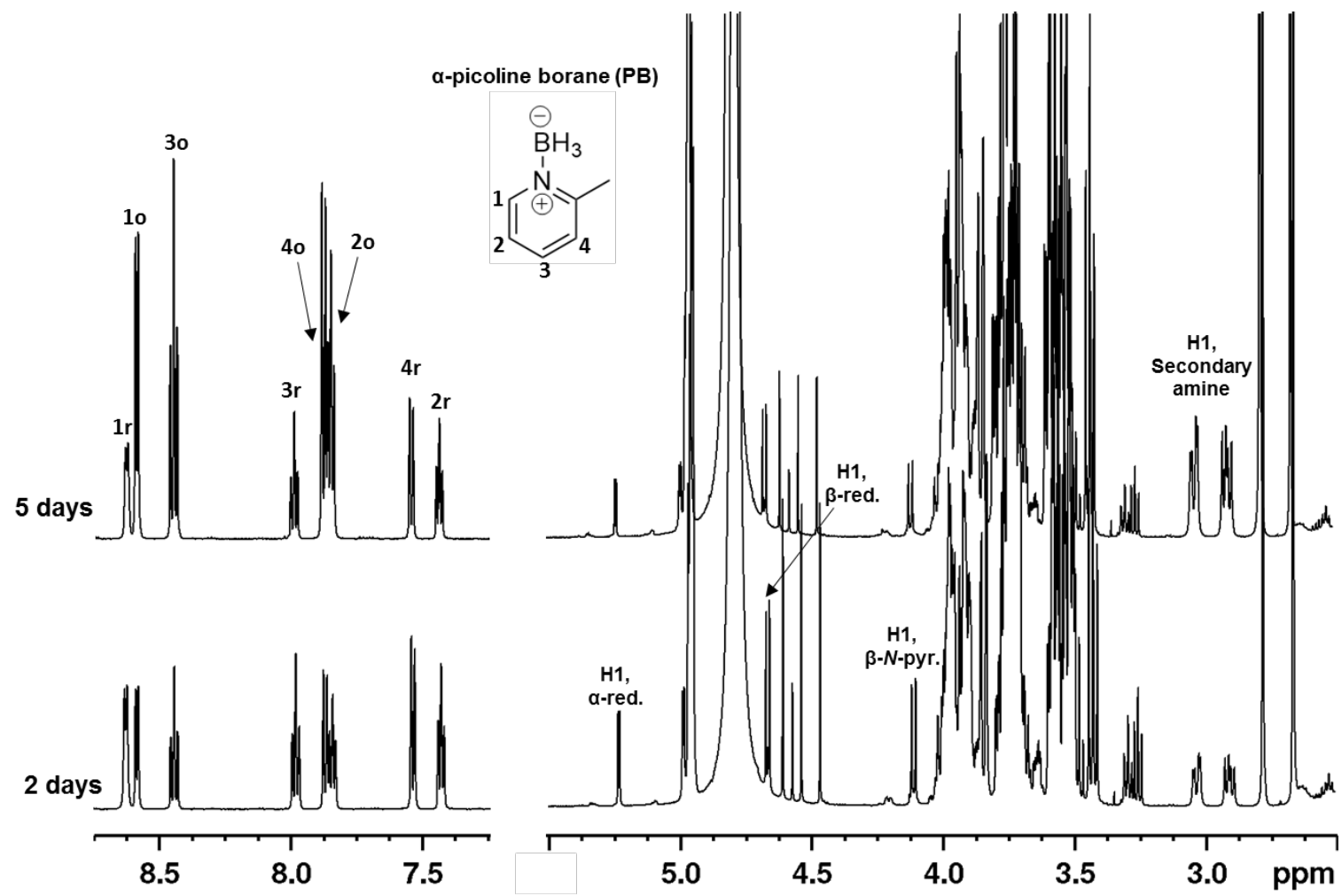

Figure S48: ${ }^{1} \mathrm{H}-\mathrm{NMR}$ characterisation of the mixture of secondary amines, conjugates and unreacted oligomers obtained after 2 and 5 days for the one pot reductive amination of Dext ${ }_{4}$ with 2 equivalents $\mathrm{ADH}$ and 3 equivalents $\mathrm{PB}$ at $\mathrm{pH}$ 4.0. The yield of secondary amines obtained after 5 days was $61 \%$. 


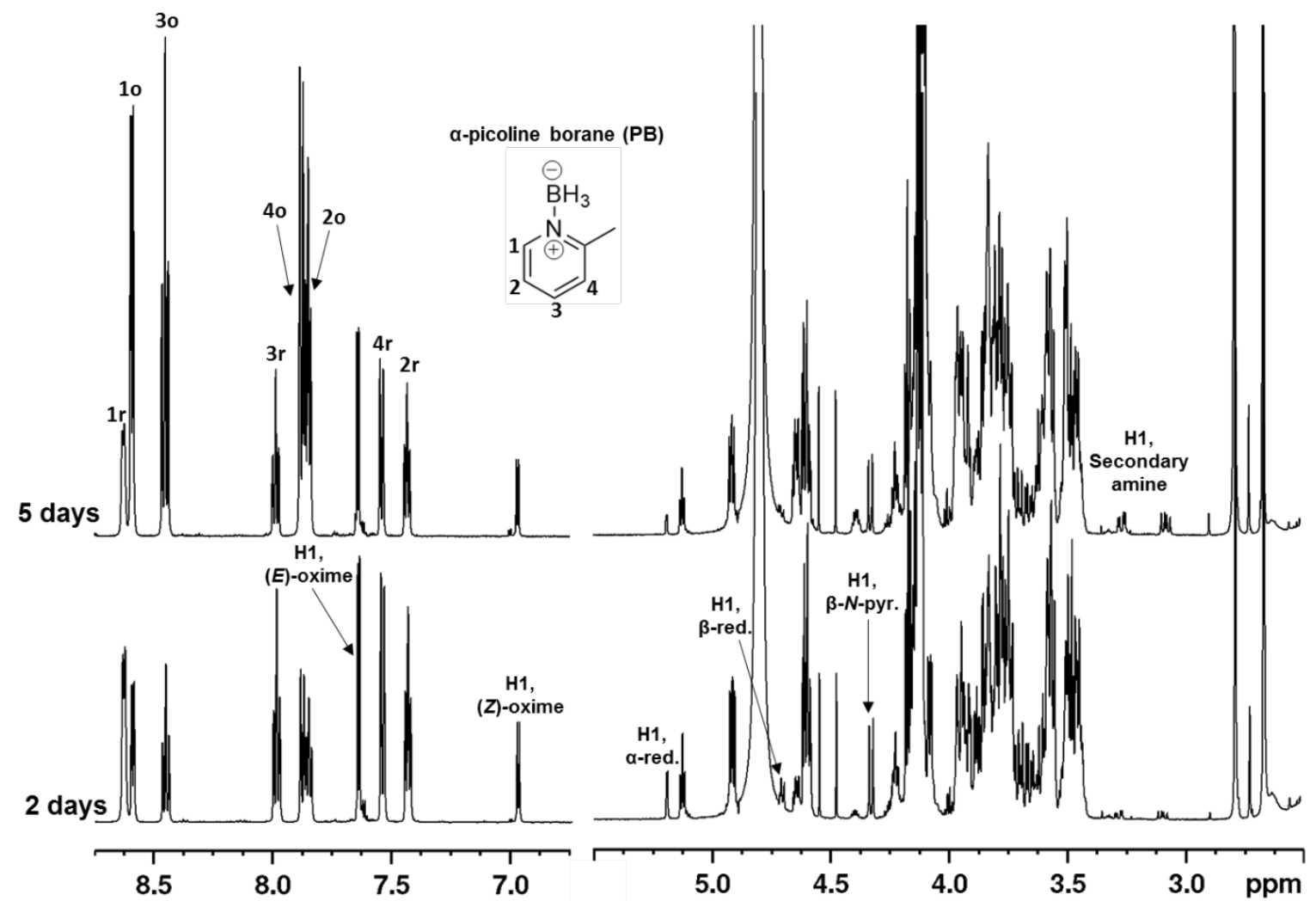

Figure S49: ${ }^{1} \mathrm{H}-\mathrm{NMR}$ characterisation of the mixture of secondary amines, conjugates and unreacted oligomers obtained after 2 and 5 days for the one pot reductive amination of AA with 2 equivalents PDHA and 3 equivalents $\mathrm{PB}$ at $\mathrm{pH}$ 4.0. The yield of secondary amines obtained after 5 days was $9 \%$.

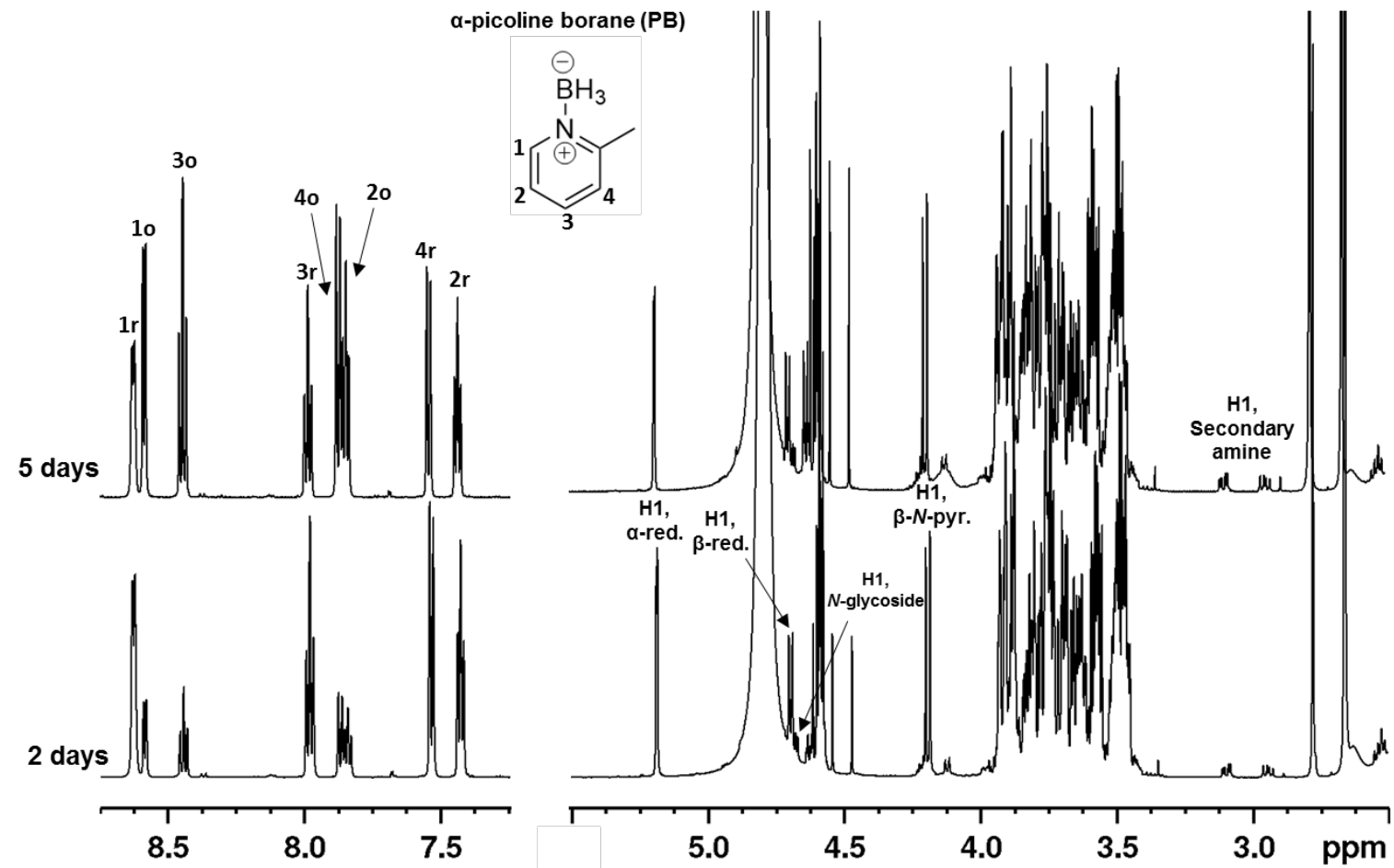

Figure S50: ${ }^{1} \mathrm{H}-\mathrm{NMR}$ characterisation of the mixture of secondary amines, conjugates and unreacted oligomers obtained after 2 and 5 days for the one pot reductive amination of AA with 2 equivalents $\mathrm{ADH}$ and 3 equivalents $\mathrm{PB}$ at $\mathrm{pH}$ 4.0. The yield of secondary amines obtained after 5 days was $6 \%$. 
Table S4. Yields (\%) obtained from the ${ }^{1} \mathrm{H}-\mathrm{NMR}$ spectra after 2 and 5 days (Figure S47-S50) for the one pot reductive amination reactions of Dext 4 and AA with 2 equivalents PDHA or ADH and 3 equivalents $\mathrm{PB}$ at $\mathrm{pH}$ 4.0.

\begin{tabular}{|c|c|c|c|c|c|c|c|}
\hline Reaction & $\begin{array}{c}\text { PDHA/ADH } \\
\text { [equivalents] }\end{array}$ & $\begin{array}{c}\text { PB } \\
\text { [equivalents] }\end{array}$ & $\begin{array}{c}\text { Time } \\
\text { [days] }\end{array}$ & $\begin{array}{c}\text { Reduced } \\
\text { conjugates } \\
{[\%]}\end{array}$ & $\begin{array}{c}\text { Non- } \\
\text { reduced } \\
\text { conjugates } \\
{[\%]}\end{array}$ & $\begin{array}{c}\text { Unreacted } \\
\text { oligomers } \\
{[\%]}\end{array}$ & $\begin{array}{l}\text { Unidentified } \\
\text { products }^{[a]} \\
{[\%]}\end{array}$ \\
\hline \multirow[b]{2}{*}{ Dext $_{4}-\mathrm{PDHA}$} & & & 2 & 31 & 64 & 5 & 0 \\
\hline & 2 & 3 & 5 & 70 & 28 & 2 & 0 \\
\hline \multirow{2}{*}{ Dext $_{4}-\mathrm{ADH}$} & & & 2 & 23 & 30 & 47 & 0 \\
\hline & 2 & 3 & 5 & 61 & 17 & 22 & 0 \\
\hline \multirow{2}{*}{ AA-PDHA } & 2 & 3 & 2 & 2 & 73 & 19 & 6 \\
\hline & & & 5 & 9 & 56 & 5 & 30 \\
\hline AA-ADH & 2 & 3 & $\begin{array}{l}2 \\
5\end{array}$ & $\begin{array}{l}4 \\
6\end{array}$ & $\begin{array}{l}37 \\
47\end{array}$ & $\begin{array}{l}58 \\
39\end{array}$ & 1 \\
\hline
\end{tabular}

[a] The yield of unidentified products was calculated as the difference between the formation of reduced (secondary amine) conjugates and the decrease in non-reduced conjugates and unreacted oligomers. Hence, yield of unidentified products $(\%)=$ theoretical yield $(100 \%)$ - yield of reduced conjugates $(\%)$ - yield of non-reduced conjugates (\%) - yield of unreacted oligomers (\%).

\section{S16 References}

Sugiyama, Hiroshi, Kanehiko Hisamichi, Kazuo Sakai, Taichi Usui, Jun-Ichi Ishiyama, Hideaki Kudo, Hiroki Ito, \& Yasuhisa Senda. (2001). The conformational study of chitin and chitosan oligomers in solution. Bioorganic \& Medicinal Chemistry, 9, 211-16.

Sørbotten, Audun, Svein J. Horn, Vincent G. H. Eijsink, \& Kjell M. Vårum. (2005). Degradation of chitosans with chitinase B from Serratia marcescens. The FEBS Journal, 272, 538-49.

van Aalten, D. M. F., D. Komander, B. Synstad, S. Gåseidnes, M. G. Peter, \& V. G. H. Eijsink. (2001). Structural insights into the catalytic mechanism of a family 18 exo-chitinase. Proceedings of the National Academy of Sciences, 98, 8979-84. 